\title{
Development of (6R)-2-Nitro-6-[4-(trifluoromethoxy)phenoxy]-6,7- dihydro-5H-imidazo[2,1-b][1,3]oxazine (DNDI-8219): A New Lead for Visceral Leishmaniasis
}

Andrew M. Thompson, ${ }^{*}{ }^{\dagger} \odot$ Patrick D. O’Connor, ${ }^{\dagger}$ Andrew J. Marshall, ${ }^{\dagger}$ Adrian Blaser, ${ }^{\dagger}$ Vanessa Yardley, ${ }^{\dagger}$ Louis Maes, ${ }^{\S}$ Suman Gupta, $\|$ Delphine Launay, ${ }^{\perp}$ Stephanie Braillard, ${ }^{\perp}$ Eric Chatelain, ${ }^{\perp}$ Baojie Wan, ${ }^{\#}$ Scott G. Franzblau, ${ }^{\#}$ Zhenkun Ma, ${ }^{@}$ Christopher B. Cooper, ${ }^{@}$ and William A. Denny ${ }^{\dagger}$

${ }^{\dagger}$ Auckland Cancer Society Research Centre, School of Medical Sciences, The University of Auckland, Private Bag 92019, Auckland 1142, New Zealand

${ }^{\ddagger}$ Faculty of Infectious \& Tropical Diseases, London School of Hygiene \& Tropical Medicine, Keppel Street, London WC1E 7HT, United Kingdom

${ }^{\S}$ Laboratory for Microbiology, Parasitology and Hygiene, Faculty of Pharmaceutical, Biomedical and Veterinary Sciences, University of Antwerp, Universiteitsplein 1, B-2610 Antwerp, Belgium

"Division of Parasitology, CSIR-Central Drug Research Institute, Lucknow 226031, India

${ }^{\perp}$ Drugs for Neglected Diseases initiative, 15 Chemin Louis Dunant, 1202 Geneva, Switzerland

\#Institute for Tuberculosis Research, College of Pharmacy, University of Illinois at Chicago, 833 South Wood Street, Chicago, Illinois 60612, United States

${ }^{\circledR}$ Global Alliance for TB Drug Development, 40 Wall Street, New York, New York 10005, United States

Supporting Information

ABSTRACT: Discovery of the potent antileishmanial effects of antitubercular 6-nitro-2,3-dihydroimidazo[2,1-b][1,3]oxazoles and 7-substituted 2-nitro-5,6-dihydroimidazo[2,1$b][1,3]$ oxazines stimulated the examination of further scaffolds (e.g., 2-nitro-5,6,7,8-tetrahydroimidazo[2,1-b][1,3]oxazepines), but the results for these seemed less attractive. Following the screening of a 900-compound pretomanid analogue library, several hits with more suitable potency, solubility, and microsomal stability were identified, and the

900 COMPOUND LIBRARY SCREEN

NEW LEAD (R-6):

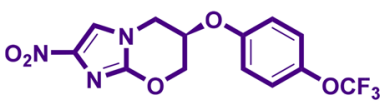

$E D_{50} 7.5 \mathrm{mg} / \mathrm{kg}$ (L. don, mouse)
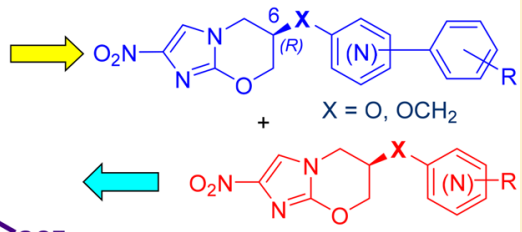

$\mathrm{X}=\mathrm{CH}_{2} \mathrm{O}, \mathrm{OCH}_{2}, \mathrm{OCH}_{2} \mathrm{C} \equiv \mathrm{C}$,

$\mathrm{O}, \mathrm{OCONH}, \mathrm{NHCH}_{2}$ $\mathrm{NHCO}, \mathrm{NHCONH}$ superior efficacy of newly synthesized $6 R$ enantiomers with phenylpyridine-based side chains was established through head-to-head assessments in a Leishmania donovani mouse model. Two such leads ( $R-84$ and $R-89)$ displayed promising activity in the more stringent Leishmania infantum hamster model but were unexpectedly found to be potent inhibitors of hERG. An extensive structure-activity relationship investigation pinpointed two compounds ( $R-6$ and pyridine $R$-136) with better solubility and pharmacokinetic properties that also provided excellent oral efficacy in the same hamster model (>97\% parasite clearance at $25 \mathrm{mg} / \mathrm{kg}$, twice daily) and exhibited minimal hERG inhibition. Additional profiling earmarked $R-6$ as the favored backup development candidate.

\section{INTRODUCTION}

Visceral leishmaniasis (VL) is a particularly lethal sandfly-borne parasitic disease that is prevalent in more than 60 countries, where it mostly affects underprivileged people in remote rural areas who have limited access to diagnosis and treatment. ${ }^{1-3}$ Major outbreaks of VL in East Africa have been attributed to waves of forced migration during periods of conflict, and such epidemics are exacerbated by weak healthcare systems, malnutrition, and HIV/AIDS coinfection., ${ }^{4,5}$ Moreover, in this region, the first-line drug combination of paromomycin and sodium stibogluconate was found to be unsuitable for $\mathrm{VL}$ patients who were $>50$ years of age or those with HIV, and no other therapies have shown adequate efficacy. ${ }^{6,7}$ Failure of the most recently evaluated new agent, fexinidazole, in a phase II clinical trial for VL in $\operatorname{Sudan}^{8}$ has now left the clinical pipeline empty, underlining the compelling need to develop more satisfactory medications. 9

The target product profile (TPP) of an optimized new chemical entity for the treatment of $\mathrm{VL}$ requires (i) effectiveness against all causative species, in all endemic areas, in both immunocompetent and immunosuppressed individuals, with a clinical efficacy of $>95 \%$; (ii) activity against resistant strains; (iii) no adverse safety events requiring monitoring and

Received: October 24, 2017

Published: February 20, 2018 
no contraindications; (iv) no drug-drug interactions (suitable for combination therapy); (v) oral administration once per day for a maximum of 10 days (or intramuscular dosing three times over 10 days); (vi) stability in relevant climates (3 years); and (vii) affordable cost $\left(<\$ 80\right.$, ideally $<\$ 10$ per course).$^{10}$ However, new drug discovery for VL faces formidable challenges, such as inadequate investment, a lack of validated targets, poor translation of in vitro activity into in vivo models, and meager hit rates $(<0.1 \%)$ for phenotypic screening of compound libraries. ${ }^{11-13}$ The latter may be due in part to the concealed location of parasites in acidic parasitophorous vacuoles within macrophages. ${ }^{14}$ Furthermore, the unique glycolipid-rich cell surface of the amastigotes presents an additional barrier to chemotherapy. ${ }^{15}$ Another issue is that many cellularly active hits may never meet TPP and progression criteria, even after valiant optimization attempts. $^{13,16}$ Nevertheless, drug development efforts spearheaded by the Drugs for Neglected Diseases initiative $(\mathrm{DND} i)$ have now shown encouraging progress in several novel classes, including oxaboroles and aminopyrazoles. ${ }^{12,17,18}$ Novartis has also disclosed a triazolopyrimidine preclinical lead with utility in vivo against both leishmanial and trypanosomal infections. ${ }^{19}$

The 2-nitroimidazooxazines are best known for their potent effects against Mycobacterium tuberculosis ( $M . t b)$, the causative agent of tuberculosis (TB). ${ }^{20}$ The first drug candidate from this class, pretomanid [PA-824, S-1 (Figure 1)], has shown
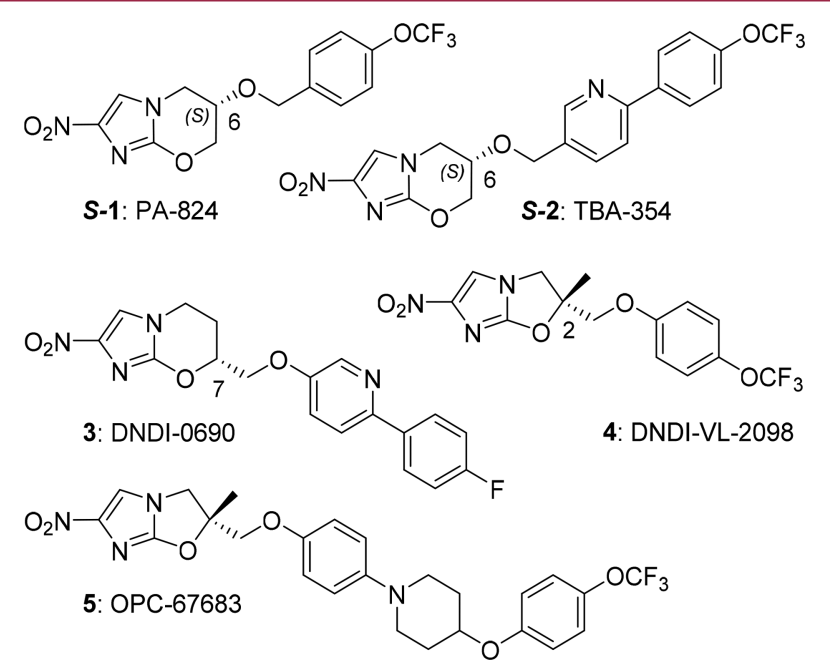

Figure 1. Structures of antitubercular or antileishmanial agents.

excellent safety and bactericidal efficacy in phase II clinical trials for $\mathrm{TB}^{21}$ leading to its ongoing combination assessment, ${ }^{22}$ while our collaborative work with the TB Alliance on second-generation analogues of $S$-1 culminated in the advancement of TBA-354 (S-2) into phase I studies. ${ }^{23}$ We have recently described the investigation of a novel 7-substituted 2nitroimidazooxazine class, which in addition to possessing considerable potential against $\mathrm{TB}$ has also demonstrated exciting activity against both VL and Chagas disease, resulting in the selection of preclinical VL lead $3 .^{24}$ This followed an indepth analysis of the structurally related 6-nitroimidazooxazole class, $^{25}$ where phenotypic screening of some of our initial examples by $\mathrm{DND} i$ had enabled the discovery of previous development nominee 4 (DNDI-VL-2098). ${ }^{26}$ The latter was found $^{27}$ to be activated by a novel leishmanial nitroreductase
(NTR2). In comparison to 4 , candidate 3 exhibited an improved safety profile and had similarly notable efficacy in two animal models of VL. ${ }^{24}$ Furthermore, while the new TB drug delamanid (5) has also been suggested as a possible VL therapy, ${ }^{28}$ it is noteworthy that 3 was substantially more effective than this agent in the highly stringent chronic infection hamster model. ${ }^{24,25}$

As part of our VL lead optimization program with DNDi, it was considered important to develop a few efficacious backup compounds having good physicochemical/pharmacological profiles and better safety, to mitigate development risks. Given the encouraging results with nitroimidazooxazoles and 7-substituted 2-nitroimidazooxazines, we first evaluated various other pretomanid-related scaffolds for VL, including those with a reversed linker at C- $6^{29,30}$ and novel nitroimidazooxazepines. We then assessed our larger collection of pretomanid analogues via the medium-throughput screening of $\sim 900$ compounds at the Institut Pasteur Korea (IPK). Finally, a more systematic synthetic approach was employed to redevelop the 6substituted 2-nitroimidazooxazine class for VL, taking into consideration both enantiomer forms. We now report the findings from these wide-ranging structure-activity relationship (SAR) studies, including the detailed in vitro/in vivo profiling of selected new leads, which resulted in our identification of the title compound as a very promising VL backup candidate.

\section{CHEMISTRY}

Scheme 1 outlines the synthetic methods used to prepare eight novel racemic analogues of $S-\mathbf{1}$ featuring changes to the original nitroimidazooxazine core $(14,22,23,32,33,39,40$, and 47). A common strategy (based on the original, well-validated route to $S-\mathbf{1}$ and simple derivatives) $)^{31,32}$ proved to be effective for the first five of these (Scheme $1 \mathrm{~A}-\mathrm{C}$ ), involving the initial reaction of functionalized epoxides $\left(9,,^{33} \mathbf{1 7}\right.$, and $\left.27^{34}\right)$ with 2,4 dinitroimidazole (8), followed by THP protection of the derived alcohols $(\mathbf{1 0}, \mathbf{1 8}$, and 28$)$. In the shorter chain cases (11 and 19), subsequent cleavage of the TBS ether (TBAF) enabled in situ annulation, whereas in the latter instance (29), oxazepine ring formation required additional treatment with a strong base $(\mathrm{NaH})$. Removal of the THP group with methanesulfonic acid and standard alkylation chemistry on alcohols 13, 21, and 31 then gave the aforementioned targets.

For the isomeric oxazepines (Scheme 1D), THP protection of alcohol $34^{24}$ and desilylation (TBAF) similarly furnished the noncyclized alcohol 36, which was ring-closed $(\mathrm{NaH}$ and $\mathrm{DMF})$ and THP-deprotected $(\mathrm{HCl})$ to produce alcohol 38. However, both final step alkylations of $\mathbf{3 8}$ unexpectedly cogenerated significant quantities $(20-36 \%)$ of the isomeric 7-substituted oxazine derivative (41 or 42$)$, together with the desired oxazepine ether (39 or 40$)$. Although this is unconfirmed, it is postulated that this rearrangement may involve an intramolecular $S_{N} A r$ reaction, with attack by the oxazepine alkoxide anion at the imidazole ring junction carbon 9a and subsequent alkylation of the released 2-nitroimidazooxazine 7 -alkoxide (but it should be emphasized that no similar rearrangements were detected during the derivatization of 13, 21, 31, or the alcohol precursor to $S-\mathbf{1}$, and that $S-\mathbf{1}$ itself has shown excellent safety and a lengthy $16-18 \mathrm{~h}$ half-life in clinical trials for $\mathrm{TB},{ }^{35}$ suggesting that 2 -nitroimidazooxazine-based VL leads would be unlikely to demonstrate an excessive reactivity toward biological nucleophiles; this is further supported by an observed tolerance of the latter ring system toward several basic nucleophiles in the chemistry reported below). The remaining 
Scheme $1^{a}$
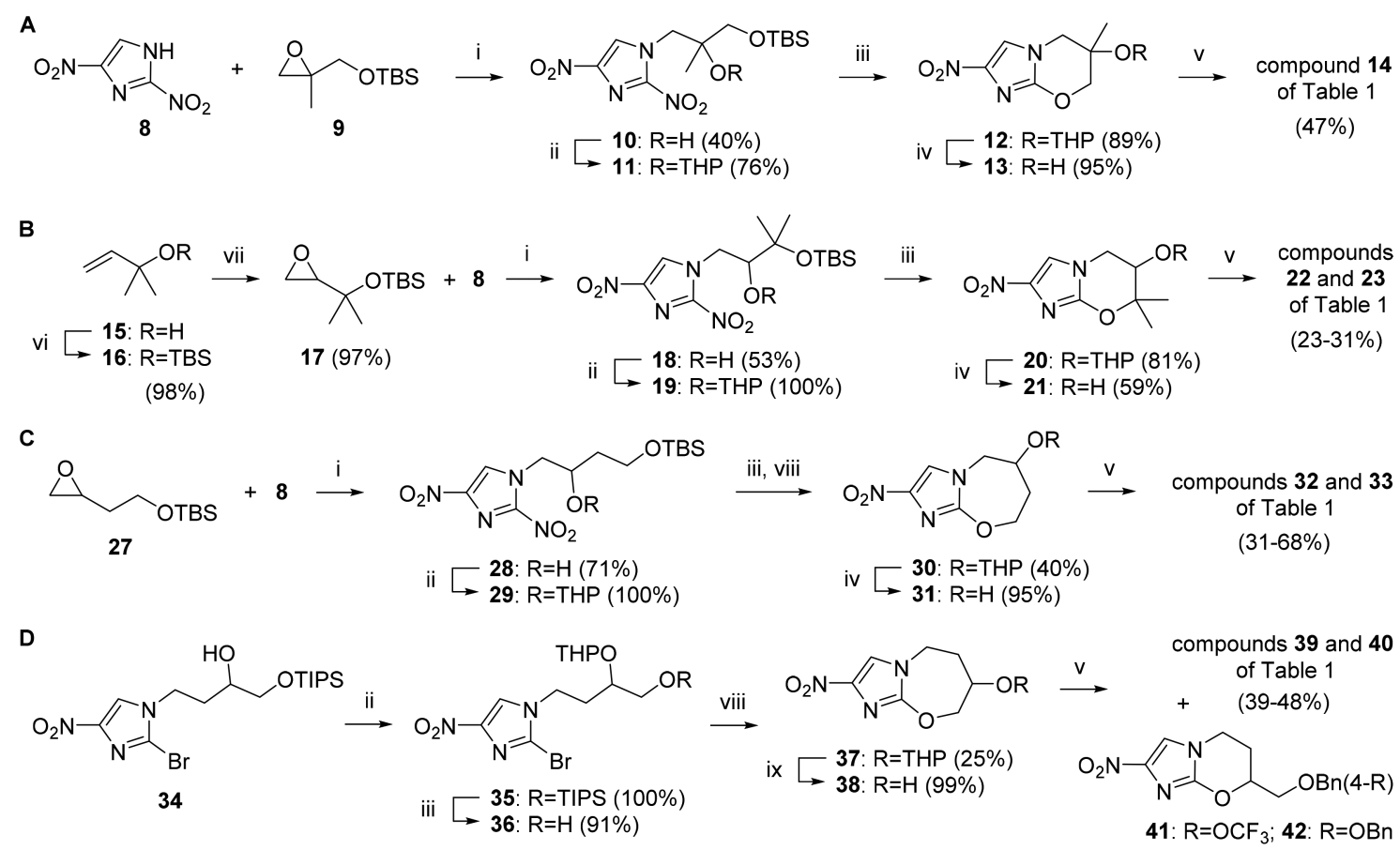

(20-36\%)

${ }^{a}$ Reagents and conditions: (i) $70-75$ or $95{ }^{\circ} \mathrm{C}, 18-23 \mathrm{~h}$; (ii) 3,4-dihydro- $2 \mathrm{H}$-pyran, PPTS, $\mathrm{CH}_{2} \mathrm{Cl}_{2}, 20{ }^{\circ} \mathrm{C}, 3.5-24 \mathrm{~h}$; (iii) TBAF, THF, $20{ }^{\circ} \mathrm{C}, 1-$ $4.5 \mathrm{~h}$, or $0-20^{\circ} \mathrm{C}, 14 \mathrm{~h}$ (for 47); (iv) $\mathrm{MsOH}, \mathrm{MeOH}, 2{ }^{\circ} \mathrm{C}, 1-2 \mathrm{~h}$; (v) 4-OCF $3 \mathrm{BnBr}$ or 4-BnOBnCl or 4-BnOBnI, NaH, DMF, $20{ }^{\circ} \mathrm{C}$ for $3-20 \mathrm{~h}$ or $0-20{ }^{\circ} \mathrm{C}$ for $0.7-2.2 \mathrm{~h}$; (vi) TBSOTf, $\mathrm{Et}_{3} \mathrm{~N}, \mathrm{CH}_{2} \mathrm{Cl}_{2}, 20^{\circ} \mathrm{C}, 3 \mathrm{~h}$; (vii) m-CPBA, $\mathrm{CH}_{2} \mathrm{Cl}_{2}, 20^{\circ} \mathrm{C}, 18 \mathrm{~h}$; (viii) $\mathrm{NaH}, \mathrm{DMF}, 20^{\circ} \mathrm{C}$ for $18 \mathrm{~h}$ or $0-20$ ${ }^{\circ} \mathrm{C}$ for $3.5 \mathrm{~h}$; (ix) $3.3 \mathrm{M} \mathrm{HCl}, \mathrm{MeOH}, 20{ }^{\circ} \mathrm{C}, 5 \mathrm{~h}$; (x) DIPEA, toluene, 89-105 ${ }^{\circ} \mathrm{C}, 67 \mathrm{~h}$.

scaffold, nitrotriazolooxazine 47, was accessed from 5-bromo-3nitro-1,2,4-triazole (43) and iodide $44,{ }^{36}$ via desilylation/in situ annulation of the major adduct 46 , as shown above (Scheme $1 \mathrm{E})$.

The assembly of new biaryl and heterobiaryl side chain variants of $S-\mathbf{1}$ and its enantiomer, $R-\mathbf{1},{ }^{37,38}$ was relatively straightforward (Schemes 2 and 3). Biphenyl analogues (R-58, $R-60$, and $R-62$ ) were created by Suzuki coupling reactions on halobenzyl ether derivatives of the key $6-R$ alcohol $56,{ }^{32}$ while combination of 56 with bromide $63^{39}$ afforded the terminal pyridine $R-64$ (Scheme 2A). Alternative alkylation of 56 or its $6-S$ equivalent $65^{32}$ with various isomeric bromomethyl bromopyridines $\left(\mathbf{6 6}, \mathbf{7 1}, \mathbf{7 5}, \mathbf{8 2},{ }^{39}\right.$ and 97$)$ then set up the Suzuki-based manufacture of novel ortho-linked phenylpyridines [S-69, R-69, S-74, and R-74 (Scheme 2B,C)] and both meta- and para-linked congeners (Schemes $2 \mathrm{D}$ and $3 \mathrm{~A}, \mathrm{C})$. This latter work was further expanded to include examples containing a pyridazine, pyrazine, or pyrimidine ring ( $R-109$, $R-112$, and $R-115)$, using similar chemistry ${ }^{39}$ (Scheme $3 D$ ), and to a novel racemic nitrotriazole counterpart of $R-84[88$ (Scheme 3B)], starting from alcohol 86. ${ }^{40}$ Lastly, a buffered $m$ CPBA oxidation of $R-84$ supplied pyridine $N$-oxide $R-85$ (Scheme 3A).

Several reference benzyl ethers $\left(R-1,{ }^{38} R-7,{ }^{32} R-118\right.$, and $R$ $119)$ were sourced through direct alkylations of $6-R$ alcohol $56^{32}$ (Scheme 4A). Three extended ether targets $(R-122, R-$ 123 , and $R-124)$ were also formed by Sonogashira reactions on the propargyl ether $R-\mathbf{1 2 1}$, derived from the coupling of $\mathbf{5 6}$ with bromide $120 .^{41}$ Next, the orthogonally diprotected triol $125^{42}$ was employed in complementary syntheses of the novel $R$ and $S$ enantiomers of racemic ether $6^{42}$ (Scheme 4B,C). Following a Mitsunobu reaction of $\mathbf{1 2 5}$ with 4(trifluoromethoxy)phenol, selective removal of the PMB group (DDQ), iodination of the resulting alcohol 127, and reaction with 2-bromo-4-nitroimidazole (129) gave silyl ether 130. Treatment of the latter with TBAF and sodium hydrideinduced ring closure then produced S-6. Conversely, successive cleavage of the TIPS group from 126, iodination, and then reaction with 129 provided $\mathrm{PMB}$ ether 134. Oxidative debenzylation of 134 with DDQ in the absence of water unexpectedly led to partial acetalization of the alcohol product with 4-methoxybenzaldehyde, but this mixture was cleanly converted back to $\mathbf{1 3 5}$ via acid hydrolysis ( $\mathrm{TsOH} / \mathrm{MeOH})$. Base-assisted annulation of alcohol $135(\mathrm{NaH})$ then furnished the second enantiomer, R-6. In subsequent work, 2-pyridinyl ether analogues of both $R-6(R-136, R-137$, and $R-138)$ and triazole 47 (139) were accessed from alcohols $56^{32}$ and $86^{40}$ respectively, via sodium hydride-catalyzed $S_{N} A r$ displacement reactions on halopyridines (Scheme 4D), while $\mathrm{Cu}(\mathrm{I})$-induced coupling of 56 with aryl isocyanates generated the $\mathrm{O}$ carbamates $R-140$ and $R-141$ (Scheme $4 \mathrm{E}$ ). Finally, piperazine carbamate $R-143$ was synthesized by chloroformylation of alcohol 56 and in situ reaction with 1-[4-(trifluoromethoxy)phenyl] piperazine $^{43}$ (142) (Scheme $4 \mathrm{~F}$ ). 
Scheme $2^{a}$

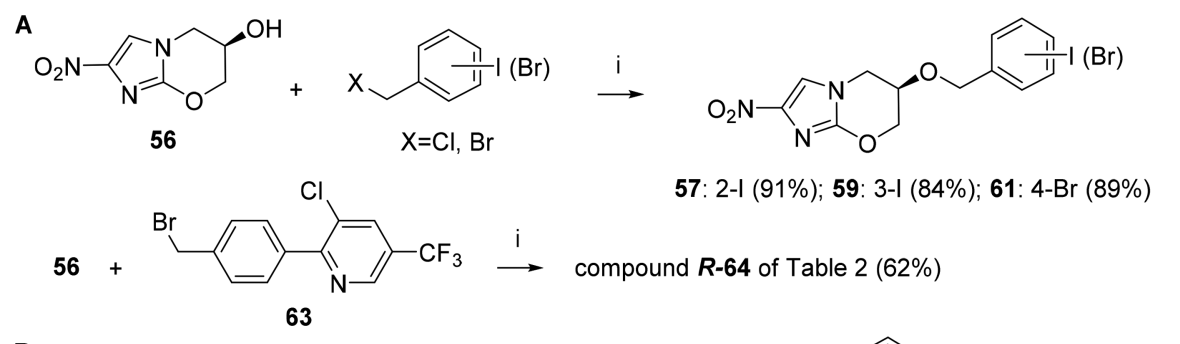

B

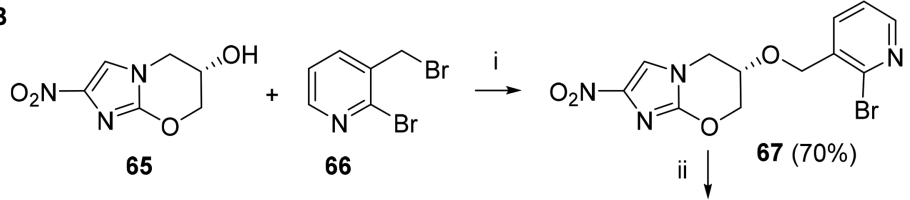

compound $\mathbf{S}-69$ of Table 2 (85\%)

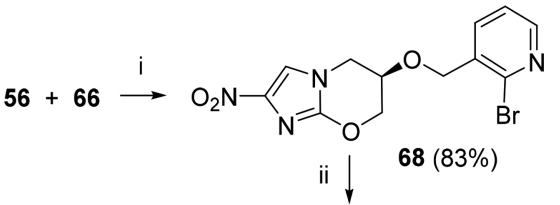

compound $\boldsymbol{R}-69$ of Table $2(84 \%)$

C<smiles>[X]Cc1ncccc1Br</smiles>

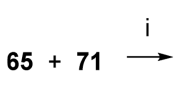<smiles></smiles>

compound S-74 of Table $2(57 \%)$

iii $\square$ 70: $\mathrm{X}=\mathrm{OH}: \mathrm{Xr}(75 \%)$

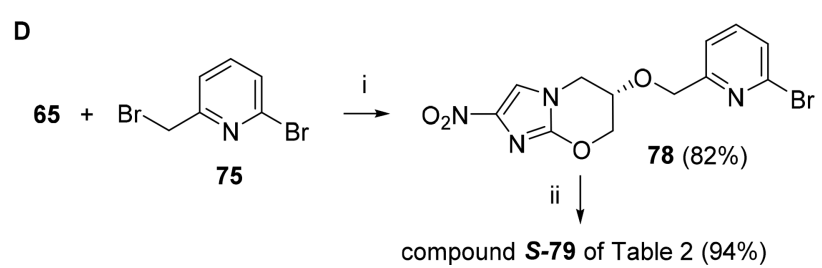

compound S-79 of Table 2 (94\%)

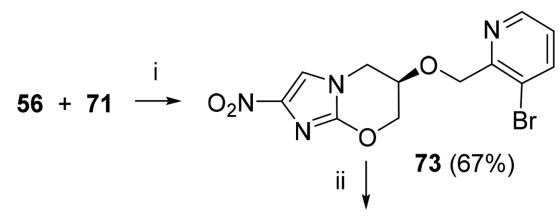

compound $\mathbf{R}-\mathbf{7 4}$ of Table 2 (50\%)

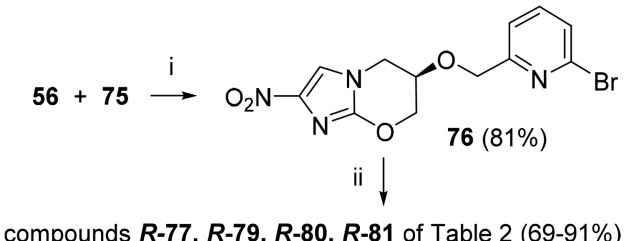

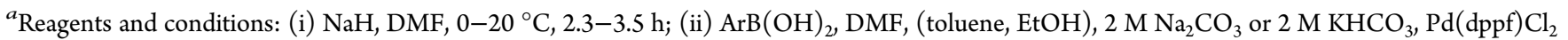
under $\mathrm{N}_{2}, 70-88{ }^{\circ} \mathrm{C}, 2.2-4 \mathrm{~h}$; (iii) NBS, $\mathrm{PPh}_{3}, \mathrm{CH}_{2} \mathrm{Cl}_{2}, 2{ }^{\circ} \mathrm{C}, 3.5 \mathrm{~h}$.

Further linker diversity was accessed through the transformation of $6-S$ alcohol $65^{32}$ into the novel $6-R$ amine hydrochloride, 146 (Scheme 5A). Following tosylation of 65 and azide displacement, reduction of $6-R$ azide 145 with propane-1,3-dithiol gave the required amine, which was converted to its hydrochloride salt for improved stability. From this intermediate, reductive alkylation with benzaldehydes (using $\mathrm{NaBH}_{3} \mathrm{CN}$ ), acylation with benzoyl chlorides, or treatment with phenyl isocyanates in the presence of catalytic dibutyltin diacetate yielded the expected benzylamine, benzamide, or phenyl urea derivatives (Scheme 5A,B). Then, to conclude this study, a variety of shorter O-linked heterobiaryl side chains were constructed using Suzuki couplings on haloheteroaryl ether precursors $[156,159,167$, 170, and 173 (Scheme 5C-E)]. The latter were obtained directly from alcohol 56, via $S_{N} A r$ reactions on fluoropyridines or chloropyrimidines, or, in the case of 167 , from the diprotected triol $125^{42}$ and 6-bromopyridin-3-ol, using the same methodology as described above for $R-6$.

\section{RESULTS AND DISCUSSION}

To establish the SARs against kinetoplastid diseases, 76 new (and several known) pretomanid analogues derived from successive projects with $\mathrm{TB}$ Alliance and $\mathrm{DND} i$ were retrospectively tested in replicate assays conducted at the University of Antwerp [LMPH (Tables 1-4)]. These assays measured activity versus the intracellular amastigote forms of both Leishmania infantum (L. inf) and Trypanosoma cruzi (T. cruzi) and against the bloodstream form of Trypanosoma brucei (T. brucei); cytotoxicity toward human lung fibroblasts (MRC-5 cells, the host for T. cruzi) was also assessed. ${ }^{44}$ Much of our VL lead optimization work with $\mathrm{DND} i$ was earlier guided by the findings from single $\mathrm{IC}_{50}$ determinations against Leishmania donovani ( $L$. don) in a mouse macrophage-based luciferase assay $^{26}$ performed at the Central Drug Research Institute (CDRI, Pradesh, India), and by follow-up evaluations of in vitro microsomal stability and efficacy in the mouse VL model (Figure 2A). The best leads were then advanced to further appraisal in the more stringent hamster VL model. Overall, while excellent in vivo efficacy was a key goal for secondgeneration VL drug candidates, we also aspired (a) to minimize compound lipophilicity (estimated using ACD $\log \mathrm{P} / \log \mathrm{D}$ software, version 14.04, Advanced Chemistry Development Inc., Toronto, ON) to lessen toxicity risks, (b) to increase aqueous solubility (as judged by kinetic data on dry powder forms of active leads) for optimal oral bioavailability, and (c) to reduce hERG inhibition potential (cf. 4) ${ }^{24}$ to improve safety.

Scaffold Modification: Initial Hits. As part of our earlier TB studies, we had examined some fundamental changes to the nitroimidazooxazine "warhead", including replacement of the nitroimidazole ring by nitropyrazole or nitrotriazole [e.g., 48-50 (Table 1)] and exchange of the 8-oxygen for sulfur or nitrogen. ${ }^{40}$ We had also explored reversal of the C-6 linker (e.g., 24-26) $)^{29,30}$ and transposition of the side chain to position 7 (e.g., 41 and 42). ${ }^{24}$ In a further extension to this work (seeking improved metabolic stability and new active 
Scheme $3^{a}$

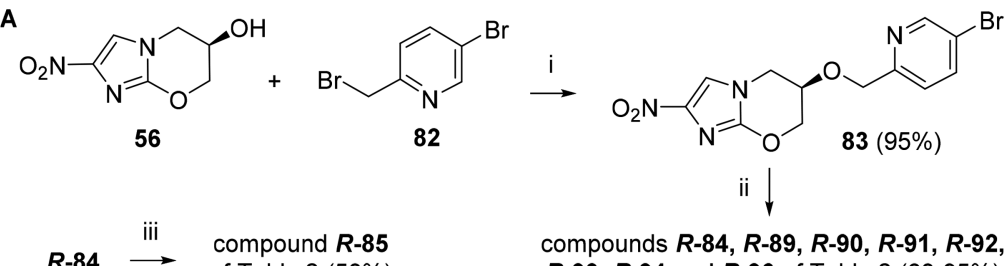

B<smiles>[13CH3]C1COc2nc([N+](=O)[O-])nn2C1</smiles>

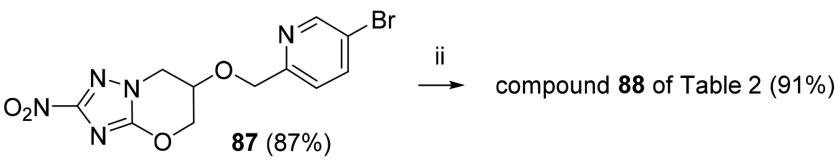

C

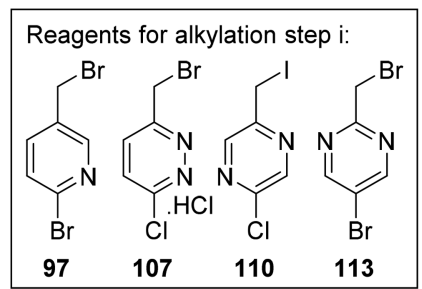

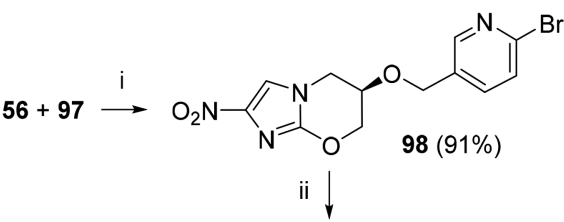

compounds $R-2, R-99, R-100, R-101, R-102$, $R$-103, $R-104$ and $R-106$ of Table $2(48-92 \%)$<smiles>O=[N+]([O-])c1cn2c(n1)OC[C@H](OCc1ccc(Br)cn1)C2</smiles>

compound S-96 of Table $2(94 \%)$ ) 
Scheme $4^{a}$

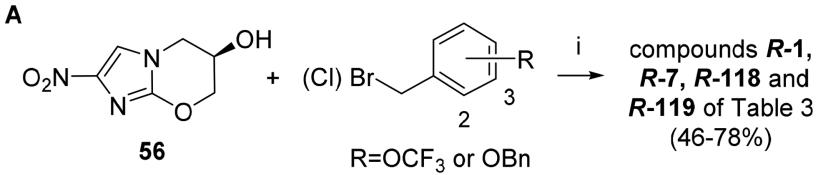

56
(46-78\%)
(4)

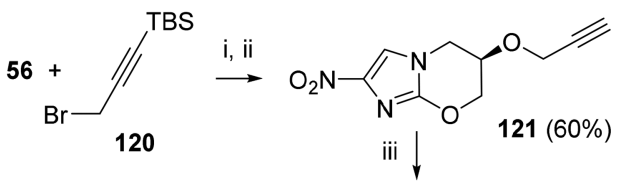

iii

compounds $\boldsymbol{R}-\mathbf{1 2 2}, \boldsymbol{R}-\mathbf{1 2 3}$ and $\boldsymbol{R}-\mathbf{1 2 4}$ of Table 3 (23-59\%)

B<smiles>[Y]C[C@H](CO[SnH3])Oc1ccc(OC(F)(F)F)cc1</smiles><smiles>[R]C[C@H](Cn1cc([N+](=O)[O-])nc1Br)Oc1ccc(OC(F)(F)F)cc1</smiles>

ii $\square$ 130: $R=$ 131: $R=H(88 \%) \quad$ i $\quad \longrightarrow \quad \begin{gathered}\text { compound } S-6 \\ \text { of Table } 3\end{gathered}$

$(70 \%)$

C
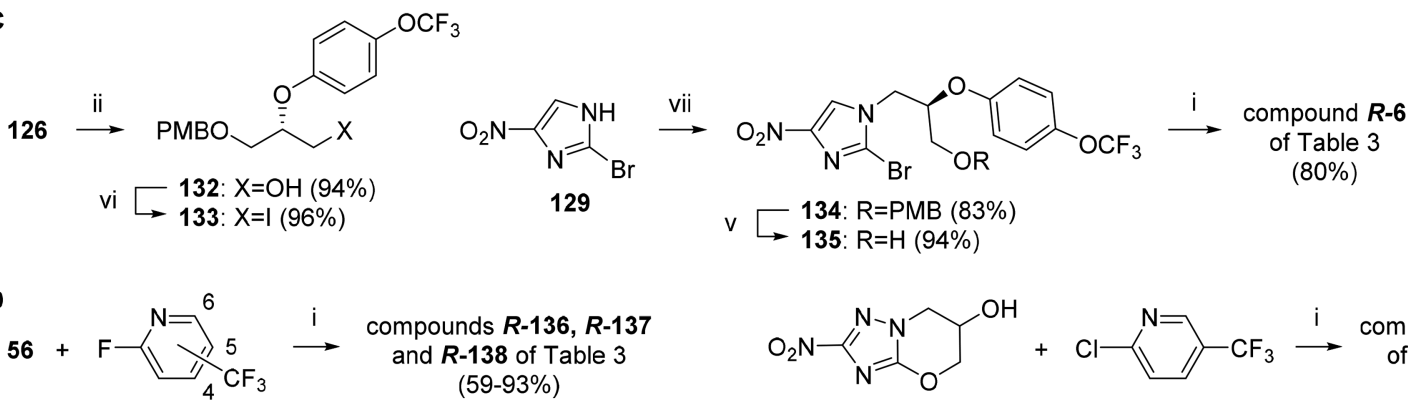
$(59-93 \%)$

E

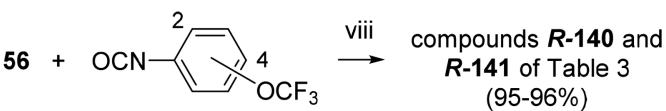

$\mathbf{F}$

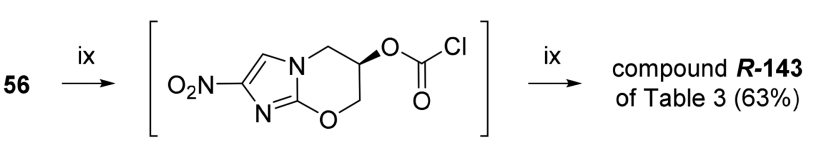

${ }^{a}$ Reagents and conditions: (i) NaH, DMF, $0-20{ }^{\circ} \mathrm{C}, 0.25-5.5 \mathrm{~h}$; (ii) TBAF, THF, $20{ }^{\circ} \mathrm{C}, 0.5-18 \mathrm{~h}$; (iii) $\mathrm{ArI}$ or $\mathrm{ArBr}, \mathrm{Et} \mathrm{H}_{3} \mathrm{~N}, \mathrm{DMF}, \mathrm{CuI}$, $\mathrm{Pd}\left(\mathrm{PPh}_{3}\right)_{2} \mathrm{Cl}_{2}$ under $\mathrm{N}_{2}, 70{ }^{\circ} \mathrm{C}$ for $0.25-1 \mathrm{~h}$ or $20^{\circ} \mathrm{C}$ for $16 \mathrm{~h}$; (iv) $4-\mathrm{OCF}_{3} \mathrm{PhOH}, \mathrm{DEAD}, \mathrm{PPh}_{3}, \mathrm{THF}, 0-20{ }^{\circ} \mathrm{C}, 60 \mathrm{~h}$; (v) $\mathrm{DDQ}^{\circ} \mathrm{CH}_{2} \mathrm{Cl}_{2}, 20{ }^{\circ} \mathrm{C}$, 10-28 h (then TsOH, $\mathrm{MeOH}, 20{ }^{\circ} \mathrm{C}, 12 \mathrm{~h}$ for 135); (vi) $\mathrm{I}_{2}, \mathrm{PPh}_{3}$, imidazole, $\mathrm{CH}_{2} \mathrm{Cl}_{2}, 20^{\circ} \mathrm{C}, 12-35 \mathrm{~h}$; (vii) 128 or $133, \mathrm{~K}_{2} \mathrm{CO}_{3}, \mathrm{DMF}^{\circ} 85-92{ }^{\circ} \mathrm{C}$, 64-111 h; (viii) $\mathrm{CuCl}, \mathrm{DMF}, 20{ }^{\circ} \mathrm{C}, 33-43 \mathrm{~h}$; (ix) triphosgene, $\mathrm{Et}_{3} \mathrm{~N}, \mathrm{THF}, 0-20{ }^{\circ} \mathrm{C}, 1.7 \mathrm{~h}$, then 142 , THF, $20{ }^{\circ} \mathrm{C}, 3.5 \mathrm{~h}$.

The most relevant results are summarized in Figure 3, with almost all of the remaining hits manifesting weaker potencies (L. don $\mathrm{IC}_{50}$ s of $\left.0.5-11 \mu \mathrm{M}\right)$. We also screened $S-\mathbf{1}$ and $S-2$, but both had only modest activities $\left(L\right.$. don $\mathrm{IC}_{50} \mathrm{~s}$ of 3.9 and 2.6 $\mu \mathrm{M}$, respectively). Nevertheless, in view of the 10 -fold higher potency of racemic 1 [ $\mathrm{IC}_{50}$ of $0.39 \mu \mathrm{M}$ (Table 1)], this result for $S-1$ was highly significant as it implied that $6 R$ enantiomers (which have little activity against $M . t b^{32,37}$ ) may be the more active chiral form for VL. Therefore, we synthesized a trial set of 10 compounds ( $R-1, R-2, R-7, R-58, R-60, R-62, R-77, R-81$, $R-84$, and $R-94)$ for assessment. In 9 of 10 cases, these $6 R$ enantiomers exhibited 1.1-12-fold superior potencies [ $L$. don $\mathrm{IC}_{50} \mathrm{~s}$ of $0.06-1.4 \mu \mathrm{M}$ (see Tables S2 and S3)]; hence, the $6 R$ counterparts of selected hits in Figure 3 were also targeted (in line with the approach in Figure 2A).

In an effort to further prioritize the library screening hits for in vivo evaluation, five compounds of high lipophilicity (CLogP $>4$ for $S-53,{ }^{46} S-54,{ }^{30} S-55,{ }^{47} S-58,{ }^{47}$ and $S-64^{39}$ ) were omitted from further study and the remaining 12 were assessed for aqueous solubility and microsomal stability (Table 5 and cited references for Figure 3). Both the amide $S-\mathbf{1 5 1}^{48}$ and urea $S$ 155 provided encouraging solubility data $(132$ and $22 \mu \mathrm{g} / \mathrm{mL}$, respectively), but the urea unexpectedly showed poor stability toward mouse liver microsomes (MLM, 43\% parent after 30 min). Conversely, both the lipophilic arylthiazole $S-51^{49}$ $(C \log \mathrm{P} \sim 4.0)$ and the arylpyrimidine $S-171^{42}$ were considered of borderline interest because of their modest solubility values $(0.9-1.6 \mu \mathrm{g} / \mathrm{mL})$. While some phenylpyridine hits (e.g., $S-77$, $S-89, S-91, S-92$, and $S-99)^{39}$ were not substantially more soluble than this at $\mathrm{pH} 7(1.4-4.0 \mu \mathrm{g} / \mathrm{mL})$, these compounds have demonstrated greatly superior results at $\mathrm{pH} 1$ (211-1780 $\mu \mathrm{g} / \mathrm{mL}$ ). It has been recognized that the low $\mathrm{pH}$ of gastric fluid (typically $\sim 1-2$ ) can enhance the dissolution and oral absorption of such weak bases. ${ }^{50}$ Furthermore, close analogue S-2 was advanced to clinical studies for TB partly on the basis of its superior in vivo $\mathrm{PK}$ properties in comparison to those of delamanid (5), which are absorption-limited. ${ }^{23}$ Concordant with this, the most potent phenylpyridine hits in Figure 3 ( $S$ 77, S-81, S-89, S-91, and $S-92)$ also displayed broadly acceptable HLM and MLM stabilities (>70\% remaining after $30 \mathrm{~min}$ ), suggesting their suitability for in vivo studies.

The best six screening hits mentioned above (phenylpyridines $S-77, S-81, S-89, S-91$, and $S-92$ and amide $S-151$ ) were then evaluated in the mouse VL model alongside a similar set of $6 R$ enantiomers $(R-77, R-84, R-89, R-91, R-92, R-94$, and $R-151)$, dosing at $50 \mathrm{mg} / \mathrm{kg}$ orally, once daily for 5 days. Results for the meta-linked phenylpyridines $R-77, S-77$, and $S$ 81 were not particularly impressive [52, 44, and 35\% inhibition, 
Scheme $5^{a}$

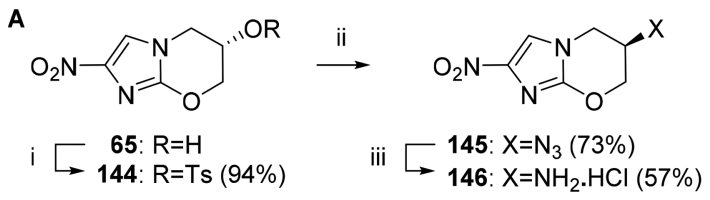

B

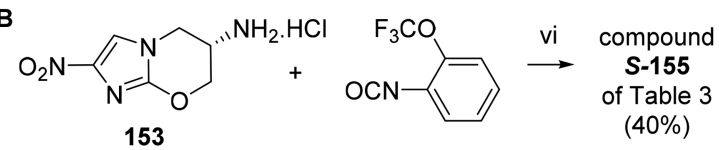

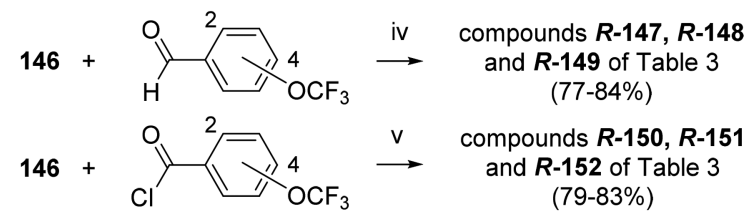

$146+\mathrm{OCN}$

C

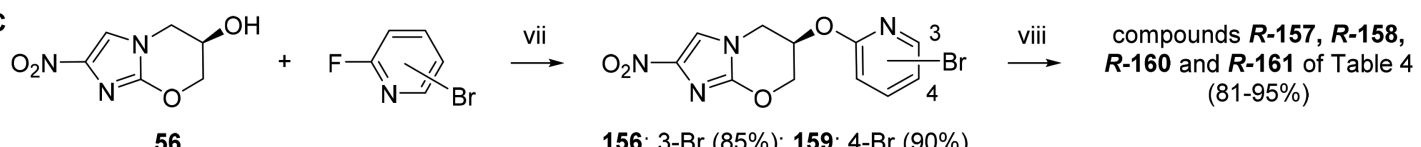

56

156: 3-Br (85\%); 159: 4-Br (90\%)

D

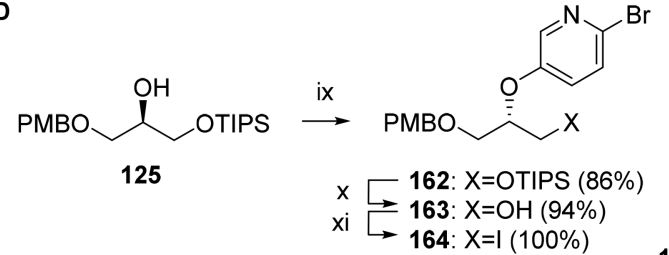<smiles>[R]CC(Cn1cc([N+](=O)[O-])nc1Br)Oc1ccc(Br)nc1</smiles>
129 xiii $\square$ 165: $R=P M B(76 \%)$ $166 \stackrel{\text { vii }}{\longrightarrow}$ 每 $\begin{gathered}\text { R-169 of Table } 4 \\ (85-86 \%)\end{gathered}$ $167(81 \%)$

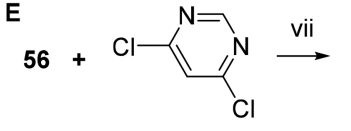<smiles>O=[N+]([O-])c1cn2c(n1)OCC(Oc1cc(Cl)ncn1)C2</smiles>

compounds $R-171$ and $R-172$ of Table 4 (88-91\%)

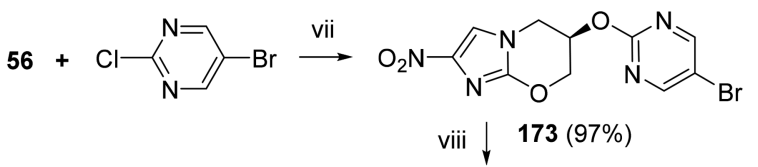

compounds $R$-174 and $R-175$ of Table 4 (97\%)

${ }^{a}$ Reagents and conditions: (i) TsCl, pyridine, $49^{\circ} \mathrm{C}, 17 \mathrm{~h}$; (ii) $\mathrm{NaN}_{3}$, DMSO, $64{ }^{\circ} \mathrm{C}, 3.5$ days; (iii) $\mathrm{HS}\left(\mathrm{CH}_{2}\right)_{3} \mathrm{SH}, \mathrm{Et}_{3} \mathrm{~N}, \mathrm{MeOH}, 20{ }^{\circ} \mathrm{C}, 0.5 \mathrm{~h}$, then $\mathrm{HCl}$, dioxane; (iv) $\mathrm{NaBH}_{3} \mathrm{CN}$, AcOH, DMF, $0-20{ }^{\circ} \mathrm{C}, 7-20 \mathrm{~h}$; (v) DIPEA, DMF, $20{ }^{\circ} \mathrm{C}, 10-25 \mathrm{~h}$; (vi) NMM or DIPEA, Bu $\mathrm{Sn}(\mathrm{OAc})_{2}, \mathrm{DMF}, 20$

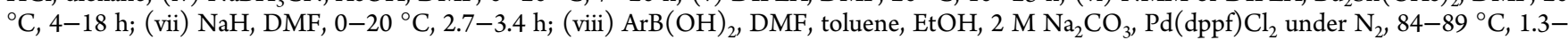
$3.5 \mathrm{~h}$; (ix) 6-bromopyridin-3-ol, DEAD, $\mathrm{PPh}_{3}$, THF, 0-20 ${ }^{\circ} \mathrm{C}$, $89 \mathrm{~h}$; (x) TBAF, THF, $20{ }^{\circ} \mathrm{C}, 13 \mathrm{~h}$; (xi) $\mathrm{I}_{2}, \mathrm{PPh}_{3}$, imidazole, $\mathrm{CH}_{2} \mathrm{Cl}_{2}, 20{ }^{\circ} \mathrm{C}, 41 \mathrm{~h}$; (xii) 164, $\mathrm{K}_{2} \mathrm{CO}_{3}, \mathrm{DMF}, 88^{\circ} \mathrm{C}, 122 \mathrm{~h}$; (xiii) $\mathrm{DDQ}, \mathrm{CH}_{2} \mathrm{Cl}_{2}, 20^{\circ} \mathrm{C}, 98 \mathrm{~h}$, then $\mathrm{TsOH}, \mathrm{MeOH}, \mathrm{CH}_{2} \mathrm{Cl}_{2}, 20{ }^{\circ} \mathrm{C}, 10 \mathrm{~h}$.

respectively (Table 5)], although the level of activity did track with their respective $L$. don $\mathrm{IC}_{50} \mathrm{~s}(0.06,0.12$, and $0.24 \mu \mathrm{M}$, respectively). In contrast, pairwise comparison of para-linked phenylpyridines $S-89, S-91$, and $S-92$ with their $6 R$ counterparts unequivocally confirmed the latter as being superior $[R-89$ (99.5\%) vs S-89 (45\%), R-91 (99.8\%) vs S-91 (69\%), and R-92 (94\%) vs S-92 (37\%)], notwithstanding their slightly higher rates of metabolism (e.g., $R$-91, $81 \%$ parent after $30 \mathrm{~min}$ in MLM, vs S-91, 100\%). In this series, the 4-fluoro analogue R-94 (L. don $\mathrm{IC}_{50}$ of $\left.0.18 \mu \mathrm{M}\right)$ showed reduced utility (83\%), whereas the apparently less potent 4-trifluoromethoxy congener $R-84$ ( $\mathrm{IC}_{50}$ of $0.32 \mu \mathrm{M}$ in the same CDRI assay) provided excellent efficacy (99.4\%), despite having lower microsomal stability ( $36 \%$ vs $66 \%$ after $1 \mathrm{~h}$ in MLM). Overall, the most effective $(6 S)$ screening hit was the soluble amide $S-151$ (72\%), but its $6 R$ form $(R-151)$ was unexpectedly poor $(5 \%)$. However, for this 6-N-linked benzamide class, it was later discovered that the $6 S$ enantiomers had the stronger in vitro potencies (e.g., L. inf $\mathrm{IC}_{50} \mathrm{~s}$ of 5.6 and $12 \mu \mathrm{M}$ for $S$-151 and $R$ 151, respectively), suggesting the need for a more systematic investigation of the SAR (Figure 2B). For better clarity, we will describe this analysis next, before summarizing the results from additional in vivo assessments on all of the most active new VL leads.
SAR of 6-Substituted 2-Nitroimidazooxazines for VL. Following the discovery that many $6 R$ enantiomers had superior in vitro and in vivo activities against $\mathrm{VL}$, a more extensive lead optimization study was initiated to develop additional backup candidates to $\mathbf{4}$ possessing an advantageous solubility, PK-PD, and safety profile. In light of the high potency of ortho-linked biphenyl hit S-58 ( $L$. don $\mathrm{IC}_{50} 0.18$ $\mu \mathrm{M})$, we first sought to establish the optimal linking position for biaryl side chains. Comparison of $R-58, R-60$, and $R-62$ in both $L$. don and L. inf assays (Table 2) unexpectedly indicated that ortho linkage was most preferred and that para linkage was least preferred. Therefore, the novel ortho-linked phenylpyridines $S-69, R-69, S-74$, and $R-74$ were studied. Here, $R$ 69 and $R-74$ were equally best, although 1.8 -fold less effective than $R-58\left(L\right.$. inf $\mathrm{IC}_{50} \mathrm{~s}$ of 2.0 vs $\left.1.1 \mu \mathrm{M}\right)$. Interestingly, these two phenylpyridine isomers showed major differences in both solubility and microsomal stability, with the more soluble $R-74$ (78 vs $0.51 \mu \mathrm{g} / \mathrm{mL}$ ) being metabolized extremely rapidly in all three microsome species $[0.1-8 \%$ remaining after $1 \mathrm{~h}$ (Table 5)], whereas R-69 was more stable than the para-linked analogue $R-84$ described above (44\% vs $36 \%$ after $1 \mathrm{~h}$ in MLM).

In the meta-linked phenylpyridine series, two new compounds ( $R-79$ and $R-80$ ) having terminal ring substituents 
Table 1. In Vitro Antiparasitic and Antitubercular Activities for Racemic Nitroheterobicyclic Scaffold Variants<smiles>O=[N+]([O-])c1cn2c(n1)OCC(O[Tl])C2</smiles><smiles>[B]c1nc([N+](=O)[O-])cn1CC(C)(O)[AlH]</smiles><smiles>Cc1nc([N+](=O)[O-])cn1CC1CC([Al])C(C)(C)O1</smiles><smiles>O=[N+]([O-])c1cn2c(n1)OCC(CO[Al])C2</smiles><smiles>O=[N+]([O-])c1cn2c(n1)OCCC(O[Al])C2</smiles><smiles>O=[N+]([O-])c1cn2c(n1)OCC([Al])CC2</smiles><smiles>O=[N+]([O-])c1cn2c(n1)OC(CO[Al])CC2</smiles><smiles>O=[N+]([O-])c1nc2n(n1)CC(O[Tl])CO2</smiles><smiles>O=[N+]([O-])c1cc2n(n1)CC(O[Tl])CO2</smiles>

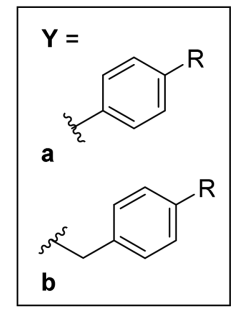

G

$\mathrm{H}$

$$
\text { I }
$$

\begin{tabular}{|c|c|c|c|c|c|c|c|c|c|c|}
\hline \multirow[b]{2}{*}{ compd } & \multirow[b]{2}{*}{ form } & \multirow[b]{2}{*}{$\mathrm{R}$} & \multirow[b]{2}{*}{ CLogP } & \multicolumn{5}{|c|}{$\mathrm{IC}_{50}^{a, b}(\mu \mathrm{M})$} & \multicolumn{2}{|c|}{$\mathrm{MIC}^{b, c}(\mu \mathrm{M})$} \\
\hline & & & & L. don & L. inf & T. cruzi & T. brucei & MRC-5 & MABA & LORA \\
\hline $6^{d}$ & Аa & $\mathrm{OCF}_{3}$ & 2.48 & 0.39 & 0.95 & 0.39 & $>64$ & $>64$ & 2.9 & 9.6 \\
\hline $1^{e}$ & $\mathrm{Ab}$ & $\mathrm{OCF}_{3}$ & 2.70 & 0.39 & 4.0 & 1.2 & $>64$ & $>64$ & 1.1 & 4.4 \\
\hline $7^{e}$ & $\mathrm{Ab}$ & OBn & 3.32 & 1.1 & 5.9 & 0.38 & $>64$ & $>64$ & 0.11 & 2.7 \\
\hline 14 & $\mathrm{Bb}$ & $\mathrm{OCF}_{3}$ & 3.24 & 78 & $>64$ & 50 & $>64$ & $>64$ & 7.4 & 55 \\
\hline 22 & $\mathrm{Cb}$ & $\mathrm{OCF}_{3}$ & 3.74 & 5.5 & 2.2 & 0.53 & 34 & $>64$ & 1.2 & 9.6 \\
\hline 23 & $\mathrm{Cb}$ & OBn & 4.36 & 0.77 & 2.5 & 0.27 & 29 & $>64$ & 2.2 & 8.3 \\
\hline $24^{f}$ & $\mathrm{Da}$ & $\mathrm{OCF}_{3}$ & 2.78 & 0.46 & 4.0 & $<0.13$ & $>64$ & $>64$ & 0.63 & 16 \\
\hline $25^{f}$ & $\mathrm{Db}$ & $\mathrm{OCF}_{3}$ & 2.77 & 2.6 & 1.7 & $<0.13$ & $>64$ & $>64$ & 2.4 & 7.9 \\
\hline $26^{f}$ & $\mathrm{Db}$ & OBn & 3.39 & 2.0 & 2.6 & $<0.13$ & $>64$ & $>64$ & 3.1 & 35 \\
\hline 32 & $\mathrm{~Eb}$ & $\mathrm{OCF}_{3}$ & 2.74 & $>2$ & $>64$ & 51 & $>64$ & $>64$ & $>128$ & $>128$ \\
\hline 33 & $\mathrm{~Eb}$ & OBn & 3.36 & $>2$ & 16 & 2.3 & 23 & $>64$ & $>128$ & $>128$ \\
\hline 39 & $\mathrm{Fb}$ & $\mathrm{OCF}_{3}$ & 2.74 & 0.63 & $>64$ & 4.2 & $>64$ & 58 & 52 & 35 \\
\hline 40 & $\mathrm{Fb}$ & OBn & 3.36 & 0.26 & $>64$ & 2.3 & 3.2 & $>64$ & $>128$ & 86 \\
\hline $41^{g}$ & $\mathrm{~Gb}$ & $\mathrm{OCF}_{3}$ & 2.88 & 0.03 & 0.12 & 1.2 & $>64$ & $>64$ & 1.0 & 7.5 \\
\hline $42^{g}$ & $\mathrm{~Gb}$ & $\mathrm{OBn}$ & 3.50 & 0.05 & & & & & 0.46 & 3.0 \\
\hline 47 & $\mathrm{Ha}$ & $\mathrm{OCF}_{3}$ & 2.51 & & $>64$ & 1.3 & $>64$ & $>64$ & & \\
\hline $48^{e}$ & $\mathrm{Hb}$ & $\mathrm{OCF}_{3}$ & 2.74 & & $>64$ & 0.73 & 38 & $>64$ & $>128$ & $>128$ \\
\hline $49^{e}$ & $\mathrm{Hb}$ & $\mathrm{OBn}$ & 3.36 & & $>64$ & 0.25 & $>64$ & $>64$ & $>128$ & $>128$ \\
\hline $50^{e}$ & $\mathrm{Ib}$ & $\mathrm{OCF}_{3}$ & 3.26 & & $>64$ & 8.2 & 46 & $>64$ & $>128$ & $>128$ \\
\hline
\end{tabular}

${ }^{a} \mathrm{IC}_{50}$ values for inhibition of the growth of $L$. don and L. inf (in mouse macrophages), T. cruzi (on MRC-5 cells), and T. brucei, or for cytotoxicity toward human lung fibroblasts (MRC-5 cells). ${ }^{b}$ Each value (except the single-test $L$. don data) is the mean of at least two independent determinations. For complete results (mean $\pm \mathrm{SD}$ ), see the Supporting Information. ${ }^{c}$ Minimum inhibitory concentration against $M$. $t b$, determined under aerobic $(\mathrm{MABA})^{59}$ or hypoxic (LORA) ${ }^{60}$ conditions. ${ }^{d_{\mathrm{TB}}}$ data from ref $42 .{ }^{e} \mathrm{~TB}$ data from ref $40 .{ }^{f_{\mathrm{TB}}}$ data from ref $30 .{ }^{g}$ Data from ref 24.

favored in para-linked isomers described above $\left(2-\mathrm{F}, 4-\mathrm{OCF}_{3}\right.$, and $\left.4-\mathrm{OCF}_{2} \mathrm{H}\right)$ were marginally more potent than $\mathrm{R}-77$ (4$\mathrm{OCF}_{3}$ ) and R-81 (4-F) [L. inf $\mathrm{IC}_{50}$ s of 0.45 and 0.51 vs 0.63 and $0.73 \mu \mathrm{M}$, respectively (Table 2$)$ ]. Here, replacement of the proximal phenyl ring in $R-60$ by 2 -pyridine $(R-77)$ resulted in a 3.7 -fold improvement in activity (L. inf $\mathrm{IC}_{50} \mathrm{~s}$ of 2.3 and 0.63 $\mu \mathrm{M})$. This enhancement by 2-pyridine was even more pronounced in the para-linked phenylpyridine series ( $L$. inf $\mathrm{IC}_{50} \mathrm{~s}$ of 0.71 and $>64 \mu \mathrm{M}$ for $R-84$ and $R-62$, respectively), clarifying that with this heterocycle, ortho linkage was less useful than meta or para linkage. However, in the para-linked set, we considered that the $2^{\prime}$-nitrogen would have less steric protection against oxidation, perhaps accounting for the modest microsomal stabilities of some analogues. Indeed, we could form the $N$-oxide derivative of $R-84(R-85)$, which was found to be 2.3 -fold less potent ( $L$. inf $\mathrm{IC}_{50}$ of $1.6 \mu \mathrm{M}$ ). Because microsomal stability varied significantly with the substituents in the terminal ring, we evaluated three new congeners (R-90, 3-F, $\left.4-\mathrm{OCF}_{3} ; \mathrm{R}-93,4-\mathrm{CF}_{3} ; \mathrm{R}-96,2,4-\mathrm{diF}\right)$. The most promising of these was $R-96\left(L\right.$. inf $\mathrm{IC}_{50}$ of $1.2 \mu \mathrm{M}$, activity $\sim 2$-fold weaker than those of early leads $R-84, R-89, R-91$, and $R-92$ ), which exhibited a better stability profile in MLM and HLM [57-59\% vs $16-40 \%$ for $R-\mathbf{8 4}, R-89$, and $R-91$ (Table 5)].
As suggested by the screening data (Figure 3), replacement of the proximal phenyl ring in R-62 with 3-pyridine ( $R-2)$ was less favorable $\left[L\right.$. inf $\mathrm{IC}_{50}$ of $4.1 \mu \mathrm{M}$ vs $0.71 \mu \mathrm{M}$ for $R-84$ (Table $2)]$. Nevertheless, the $6 R$ enantiomers of two hits $(R-99,2-F, 4-$ $\left.\mathrm{OCF}_{3} ; \mathrm{R}-101,2-\mathrm{Cl}, 4-\mathrm{OCF}_{3}\right)$ and the novel 2,4-difluoro analogue $R-106$ all displayed good potencies ( $L$. inf $\mathrm{IC}_{50} \mathrm{~s}$ of $1.1,0.61$, and $0.85 \mu \mathrm{M}$, respectively) and microsomal stabilities at least comparable to those of their 2-pyridine counterparts, although R-101 was cytotoxic (MRC-5 $\mathrm{IC}_{50}$ of $17 \mu \mathrm{M}$ ). By extension, we examined three less lipophilic diaza proximal rings $(R-109, R-112$, and $R-115)$ that had proven to be very effective in our TB studies, ${ }^{39}$ but these turned out to be of less interest $\left(L\right.$. inf $\mathrm{IC}_{50} \mathrm{~S}$ of 1.4-2.8 $\left.\mu \mathrm{M}\right)$ ). In summary, several new phenylpyridines provided profiles that were attractive for in vivo evaluation, but we had yet to investigate other linker groups. Therefore, we next turned our attention to simpler monoaryl side chains to explore these changes. For this part of the study, we restricted our focus to linkers that had shown particular promise either in the initial screening (e.g., 6-O, 6-NHCO, and $6-\mathrm{NHCONH}$ ) or in our earlier TB work.

Commencing with the enantiomer of pretomanid $[R-1, L$. inf $\mathrm{IC}_{50}$ of $4.7 \mu \mathrm{M}$ (Table 3 )], we found variation of the trifluoromethoxy position identified that ortho substitution was 
Table 2. In Vitro Antiparasitic Activities of 6- $\mathrm{OCH}_{2} / \mathrm{CH}_{2} \mathrm{O}$-Linked Biaryl Nitroimidazooxazines

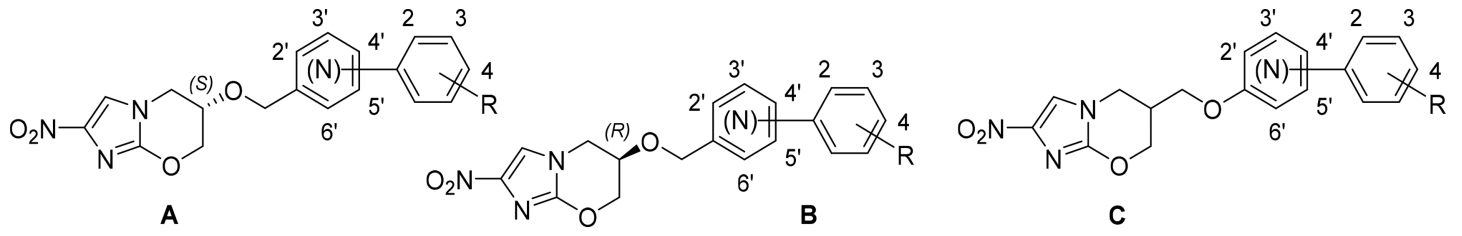

\begin{tabular}{|c|c|c|c|c|c|c|c|c|c|c|}
\hline \multirow[b]{2}{*}{ compd } & \multirow[b]{2}{*}{ form } & \multirow[b]{2}{*}{ link } & \multirow[b]{2}{*}{ aza } & \multirow[b]{2}{*}{$\mathrm{R}$} & \multirow[b]{2}{*}{ CLogP } & \multicolumn{5}{|c|}{$\mathrm{IC}_{50}^{a, b}(\mu \mathrm{M})$} \\
\hline & & & & & & L. don & L. inf & T. cruzi & T. brucei & MRC-5 \\
\hline$S-51^{c}$ & & & & & 3.97 & 0.25 & 2.3 & 4.2 & $>64$ & $>64$ \\
\hline$S-52^{c}$ & & & & & 2.60 & 0.40 & 5.2 & 4.9 & $>64$ & $>64$ \\
\hline$S-58^{d}$ & A & $2^{\prime}$ & & $4-\mathrm{OCF}_{3}$ & 4.36 & 0.18 & 30 & 0.88 & $>64$ & $>64$ \\
\hline$R-58$ & B & $2^{\prime}$ & & $4-\mathrm{OCF}_{3}$ & 4.36 & 0.17 & 1.1 & 0.64 & $>64$ & $>64$ \\
\hline$R-60$ & B & $3^{\prime}$ & & $4-\mathrm{OCF}_{3}$ & 4.36 & 0.31 & 2.3 & 0.43 & 22 & 62 \\
\hline$R-62$ & B & $4^{\prime}$ & & $4-\mathrm{OCF}_{3}$ & 4.36 & 1.2 & $>64$ & 0.12 & $>64$ & $>64$ \\
\hline$S-64^{e}$ & A & $4^{\prime}$ & 2 & $4-\mathrm{CF}_{3}, 6-\mathrm{Cl}$ & 4.46 & 0.13 & 1.5 & 3.3 & 54 & $>64$ \\
\hline$R-64$ & B & $4^{\prime}$ & 2 & $4-\mathrm{CF}_{3}, 6-\mathrm{Cl}$ & 4.46 & 0.21 & 0.87 & 0.65 & 43 & 57 \\
\hline$S-69$ & A & $2^{\prime}$ & $3^{\prime}$ & $4-\mathrm{OCF}_{3}$ & 3.01 & 28 & $>64$ & 6.7 & $>64$ & $>64$ \\
\hline$R-69$ & B & $2^{\prime}$ & $3^{\prime}$ & $4-\mathrm{OCF}_{3}$ & 3.01 & 0.78 & 2.0 & 1.9 & $>64$ & $>64$ \\
\hline$S-74$ & A & $2^{\prime}$ & $6^{\prime}$ & $4-\mathrm{OCF}_{3}$ & 3.04 & 4.8 & 9.1 & 6.3 & $>64$ & $>64$ \\
\hline$R-74$ & B & $2^{\prime}$ & $6^{\prime}$ & $4-\mathrm{OCF}_{3}$ & 3.04 & 0.86 & 2.0 & 1.3 & 18 & $>64$ \\
\hline$S-77^{e}$ & A & $3^{\prime}$ & $2^{\prime}$ & $4-\mathrm{OCF}_{3}$ & 3.01 & 0.12 & 1.9 & 2.0 & $>64$ & 47 \\
\hline$R-77$ & B & $3^{\prime}$ & $2^{\prime}$ & $4-\mathrm{OCF}_{3}$ & 3.01 & 0.06 & 0.63 & 0.25 & 46 & 61 \\
\hline$S-79$ & A & $3^{\prime}$ & $2^{\prime}$ & $2-\mathrm{F}, 4-\mathrm{OCF}_{3}$ & 3.57 & & 2.4 & 3.8 & $>64$ & $>64$ \\
\hline$R-79$ & B & $3^{\prime}$ & $2^{\prime}$ & $2-\mathrm{F}, 4-\mathrm{OCF}_{3}$ & 3.57 & & 0.45 & 0.56 & $>64$ & $>64$ \\
\hline$R-80$ & B & $3^{\prime}$ & $2^{\prime}$ & $4-\mathrm{OCF}_{2} \mathrm{H}$ & 2.16 & & 0.51 & 0.26 & $>64$ & $>64$ \\
\hline$S-81^{e}$ & A & $3^{\prime}$ & $2^{\prime}$ & $4-\mathrm{F}$ & 2.10 & 0.24 & 32 & 3.9 & $>64$ & $>64$ \\
\hline$R-81$ & B & $3^{\prime}$ & $2^{\prime}$ & $4-\mathrm{F}$ & 2.10 & 0.12 & 0.73 & 0.36 & $>64$ & $>64$ \\
\hline$S-84^{e}$ & A & $4^{\prime}$ & $2^{\prime}$ & $4-\mathrm{OCF}_{3}$ & 3.04 & 0.83 & 10 & 1.4 & 43 & $>64$ \\
\hline$R-84$ & B & $4^{\prime}$ & $2^{\prime}$ & $4-\mathrm{OCF}_{3}$ & 3.04 & $(0.24)^{f}$ & 0.71 & 0.043 & $>64$ & $>64$ \\
\hline$S-85^{g}$ & A & $4^{\prime}$ & $2^{\prime}-\mathrm{O}^{h}$ & $4-\mathrm{OCF}_{3}$ & 0.94 & & 9.1 & 5.4 & 11 & $>64$ \\
\hline$R-85$ & B & $4^{\prime}$ & $2^{\prime}-\mathrm{O}^{h}$ & $4-\mathrm{OCF}_{3}$ & 0.94 & & 1.6 & 3.3 & $>64$ & $>64$ \\
\hline $88^{i}$ & & & & & 3.08 & & 41 & 0.52 & 3.5 & 14 \\
\hline$S-89^{e}$ & A & $4^{\prime}$ & $2^{\prime}$ & $2-\mathrm{F}, 4-\mathrm{OCF}_{3}$ & 3.60 & 0.18 & 14 & 1.8 & $>64$ & $>64$ \\
\hline$R-89$ & B & $4^{\prime}$ & $2^{\prime}$ & $2-\mathrm{F}, 4-\mathrm{OCF}_{3}$ & 3.60 & $(0.27)^{f}$ & 0.62 & 0.078 & $>64$ & $>64$ \\
\hline$R-90$ & B & $4^{\prime}$ & $2^{\prime}$ & $3-\mathrm{F}, 4-\mathrm{OCF}_{3}$ & 3.02 & & 3.5 & 0.025 & $>64$ & $>64$ \\
\hline$S-91^{e}$ & A & $4^{\prime}$ & $2^{\prime}$ & $2-\mathrm{Cl}, 4-\mathrm{OCF}_{3}$ & 3.75 & 0.16 & 2.4 & 2.5 & 35 & 20 \\
\hline$R-91$ & B & $4^{\prime}$ & $2^{\prime}$ & $2-\mathrm{Cl}, 4-\mathrm{OCF}_{3}$ & 3.75 & $(0.31)^{f}$ & 0.57 & 0.12 & 35 & 41 \\
\hline$S-92^{e}$ & A & $4^{\prime}$ & $2^{\prime}$ & $4-\mathrm{OCF}_{2} \mathrm{H}$ & 2.19 & 0.08 & 3.4 & 1.2 & 46 & $>64$ \\
\hline$R-92$ & B & $4^{\prime}$ & $2^{\prime}$ & $4-\mathrm{OCF}_{2} \mathrm{H}$ & 2.19 & 0.86 & 0.63 & 0.078 & $>64$ & $>64$ \\
\hline$R-93$ & B & $4^{\prime}$ & $2^{\prime}$ & $4-\mathrm{CF}_{3}$ & 3.17 & & 6.1 & 0.072 & $>64$ & $>64$ \\
\hline$R-94$ & B & $4^{\prime}$ & $2^{\prime}$ & 4-F & 2.13 & 0.18 & 2.3 & 0.16 & $>64$ & $>64$ \\
\hline$S-96$ & A & $4^{\prime}$ & $2^{\prime}$ & 2,4-diF & 2.67 & & 1.4 & 7.8 & $>64$ & $>64$ \\
\hline$R-96$ & B & $4^{\prime}$ & $2^{\prime}$ & 2,4-diF & 2.67 & $(0.39)^{f}$ & 1.2 & 0.23 & 55 & $>64$ \\
\hline$R-2$ & B & $4^{\prime}$ & $3^{\prime}$ & $4-\mathrm{OCF}_{3}$ & 3.01 & 1.4 & 4.1 & 0.27 & $>64$ & $>64$ \\
\hline$S-99^{e}$ & A & $4^{\prime}$ & $3^{\prime}$ & $2-\mathrm{F}, 4-\mathrm{OCF}_{3}$ & 3.57 & 0.33 & $>64$ & 1.4 & $>64$ & $>64$ \\
\hline$R-99$ & B & $4^{\prime}$ & $3^{\prime}$ & $2-\mathrm{F}, 4-\mathrm{OCF}_{3}$ & 3.57 & $(0.48)^{f}$ & 1.1 & 0.19 & $>64$ & $>64$ \\
\hline$R-100$ & B & $4^{\prime}$ & $3^{\prime}$ & $3-\mathrm{F}, 4-\mathrm{OCF}_{3}$ & 2.99 & & 12 & 0.24 & $>64$ & $>64$ \\
\hline$S-101^{e}$ & A & $4^{\prime}$ & $3^{\prime}$ & $2-\mathrm{Cl}, 4-\mathrm{OCF}_{3}$ & 3.72 & 0.34 & 5.2 & 2.4 & 23 & 20 \\
\hline$R-101$ & B & $4^{\prime}$ & $3^{\prime}$ & $2-\mathrm{Cl}, 4-\mathrm{OCF}_{3}$ & 3.72 & & 0.61 & 0.15 & 34 & 17 \\
\hline$R-102$ & B & $4^{\prime}$ & $3^{\prime}$ & $4-\mathrm{OCF}_{2} \mathrm{H}$ & 2.16 & & 1.9 & 0.56 & $>64$ & $>64$ \\
\hline$R-103$ & B & $4^{\prime}$ & $3^{\prime}$ & $4-\mathrm{CF}_{3}$ & 3.14 & & 11 & 0.64 & $>64$ & $>64$ \\
\hline$R-104$ & B & $4^{\prime}$ & $3^{\prime}$ & $4-\mathrm{F}$ & 2.10 & & 1.9 & 1.7 & $>64$ & $>64$ \\
\hline$S-106$ & A & $4^{\prime}$ & $3^{\prime}$ & 2,4-diF & 2.64 & & 5.3 & 51 & $>64$ & $>64$ \\
\hline$R-106$ & B & $4^{\prime}$ & $3^{\prime}$ & 2,4-diF & 2.64 & $(0.64)^{f}$ & 0.85 & 1.3 & $>64$ & $>64$ \\
\hline$R-109$ & B & $4^{\prime}$ & $2^{\prime}, 3^{\prime}$ & $4-\mathrm{OCF}_{3}$ & 1.52 & & 2.3 & 0.55 & $>64$ & $>64$ \\
\hline$R-112$ & B & $4^{\prime}$ & $2^{\prime}, 5^{\prime}$ & $4-\mathrm{OCF}_{3}$ & 2.19 & & 2.8 & 0.27 & $>64$ & $>64$ \\
\hline$R-115$ & B & $4^{\prime}$ & $2^{\prime}, 6^{\prime}$ & $4-\mathrm{OCF}_{3}$ & 2.63 & & 1.4 & 0.28 & $>64$ & $>64$ \\
\hline $116^{g}$ & $\mathrm{C}$ & $4^{\prime}$ & $3^{\prime}$ & $4-\mathrm{OCF}_{3}$ & 3.38 & 0.05 & 6.1 & $<0.13$ & 0.63 & $>64$ \\
\hline $117^{g}$ & C & $4^{\prime}$ & $3^{\prime}$ & $4-\mathrm{F}$ & 2.46 & 0.02 & 6.3 & $<0.13$ & 17 & $>64$ \\
\hline
\end{tabular}

${ }^{a} \mathrm{IC}_{50}$ values for inhibition of the growth of $L$. don and $L$. inf (in mouse macrophages), T. cruzi (on MRC-5 cells), and T. brucei, or for cytotoxicity toward human lung fibroblasts (MRC-5 cells). ${ }^{b}$ Each value (except the single-test $L$. don data) is the mean of at least two independent 


\section{Table 2. continued}

determinations. For complete results (mean \pm SD), see the Supporting Information. ${ }^{c}$ From ref $49 .{ }^{d}$ From ref $47 .{ }^{e}$ From ref $39 .{ }^{f} \mathrm{LMPH}$ data $(\mathrm{mean}$ of three or four values). ${ }^{g}$ From ref $30 .{ }^{h} \mathrm{~N}$-Oxide. ${ }^{i}$ Racemic nitrotriazolooxazine analogue of $R-84$.

Table 3. In Vitro Antiparasitic Activities of Variously Linked Monoaryl Nitroimidazooxazines

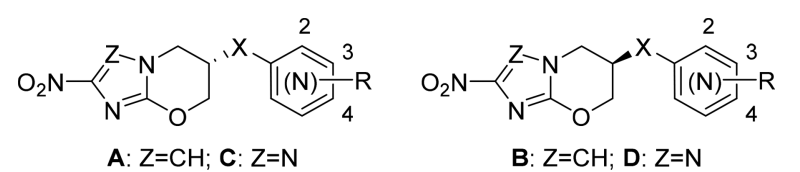

\begin{tabular}{|c|c|c|c|c|c|c|c|c|c|c|}
\hline \multirow[b]{2}{*}{ compd } & \multirow[b]{2}{*}{ form } & \multirow[b]{2}{*}{$\mathrm{X}$} & \multirow[b]{2}{*}{ aza } & \multirow[b]{2}{*}{$\mathrm{R}$} & \multirow[b]{2}{*}{ CLogP } & \multicolumn{5}{|c|}{$\mathrm{IC}_{50}^{a, b}(\mu \mathrm{M})$} \\
\hline & & & & & & L. don & L. inf & T. cruzi & T. brucei & MRC-5 \\
\hline$S-1^{c}$ & A & $\mathrm{OCH}_{2}$ & & $4-\mathrm{OCF}_{3}$ & 2.70 & 3.9 & 59 & 10 & $>64$ & $>64$ \\
\hline$R-1^{d}$ & B & $\mathrm{OCH}_{2}$ & & $4-\mathrm{OCF}_{3}$ & 2.70 & 0.54 & 4.7 & 0.40 & $>64$ & $>64$ \\
\hline$R-118$ & B & $\mathrm{OCH}_{2}$ & & $3-\mathrm{OCF}_{3}$ & 2.70 & & 2.5 & 0.31 & $>64$ & $>64$ \\
\hline$R-119$ & B & $\mathrm{OCH}_{2}$ & & $2-\mathrm{OCF}_{3}$ & 2.70 & & 1.4 & 0.20 & $>64$ & $>64$ \\
\hline$S-48^{e}$ & $\mathrm{C}$ & $\mathrm{OCH}_{2}$ & & $4-\mathrm{OCF}_{3}$ & 2.74 & & $>64$ & 0.74 & $>64$ & $>64$ \\
\hline$R-48^{e}$ & $\mathrm{D}$ & $\mathrm{OCH}_{2}$ & & $4-\mathrm{OCF}_{3}$ & 2.74 & & $>64$ & 0.54 & $>64$ & $>64$ \\
\hline$S-7^{c}$ & A & $\mathrm{OCH}_{2}$ & & $4-\mathrm{OBn}$ & 3.32 & 1.7 & $>64$ & 51 & $>64$ & $>64$ \\
\hline$R-7^{c}$ & B & $\mathrm{OCH}_{2}$ & & $4-\mathrm{OBn}$ & 3.32 & 0.14 & 0.87 & 0.39 & $>64$ & $>64$ \\
\hline$R-122$ & B & $\mathrm{OCH}_{2} \mathrm{C} \equiv \mathrm{C}$ & & $4-\mathrm{OCF}_{3}$ & 3.94 & & 0.33 & 0.11 & $>64$ & $>64$ \\
\hline$R-123$ & B & $\mathrm{OCH}_{2} \mathrm{C} \equiv \mathrm{C}$ & 2 & $4-\mathrm{CF}_{3}$ & 2.47 & & 0.44 & 1.4 & 21 & 28 \\
\hline$R-124$ & B & $\mathrm{OCH}_{2} \mathrm{C} \equiv \mathrm{C}$ & 3 & $4-\mathrm{CF}_{3}$ & 2.47 & & 0.53 & 1.2 & $>64$ & $>64$ \\
\hline$S-6$ & A & $\mathrm{O}$ & & $4-\mathrm{OCF}_{3}$ & 2.48 & & 8.2 & 7.3 & $>64$ & $>64$ \\
\hline$R-6$ & B & $\mathrm{O}$ & & $4-\mathrm{OCF}_{3}$ & 2.48 & $(0.19)^{f}$ & 0.53 & 0.15 & $>64$ & $>64$ \\
\hline$R-136$ & B & $\mathrm{O}$ & 2 & $4-\mathrm{CF}_{3}$ & 2.33 & $(0.15)^{f}$ & 1.1 & 0.34 & 25 & $>64$ \\
\hline$R-137$ & B & $\mathrm{O}$ & 2 & $3-\mathrm{CF}_{3}$ & 2.13 & & 1.2 & 0.35 & $>64$ & $>64$ \\
\hline$R-138$ & B & $\mathrm{O}$ & 2 & $5-\mathrm{CF}_{3}$ & 1.73 & & 0.85 & 2.4 & 23 & $>64$ \\
\hline $139^{g}$ & & & & & 2.37 & & $>64$ & 23 & $>64$ & $>64$ \\
\hline$R-24^{h}$ & A & $\mathrm{CH}_{2} \mathrm{O}$ & & $4-\mathrm{OCF}_{3}$ & 2.78 & 0.13 & 0.86 & 0.33 & 57 & $>64$ \\
\hline$S-24^{h}$ & B & $\mathrm{CH}_{2} \mathrm{O}$ & & $4-\mathrm{OCF}_{3}$ & 2.78 & 0.11 & 2.2 & $<0.13$ & $>64$ & $>64$ \\
\hline$S-140^{c}$ & A & OCONH & & $4-\mathrm{OCF}_{3}$ & 2.11 & 3.3 & 7.3 & 6.8 & $>64$ & $>64$ \\
\hline$R-140$ & B & OCONH & & $4-\mathrm{OCF}_{3}$ & 2.11 & & 2.1 & 3.5 & 48 & $>64$ \\
\hline$R-141$ & B & OCONH & & $2-\mathrm{OCF}_{3}$ & 2.51 & & 6.4 & 1.5 & $>64$ & $>64$ \\
\hline$S-143^{i}$ & A & OCOpip $^{j}$ & & $4-\mathrm{OCF}_{3}$ & 1.56 & 0.88 & 14 & 2.1 & $>64$ & $>64$ \\
\hline$R-143$ & B & OCOpip $^{j}$ & & $4-\mathrm{OCF}_{3}$ & 1.56 & & 17 & 0.27 & $>64$ & $>64$ \\
\hline$S-147^{i}$ & A & $\mathrm{NHCH}_{2}$ & & $4-\mathrm{OCF}_{3}$ & 2.26 & 6.1 & $>64$ & 11 & 3.6 & $>64$ \\
\hline$R-147$ & B & $\mathrm{NHCH}_{2}$ & & $4-\mathrm{OCF}_{3}$ & 2.26 & & 12 & 1.8 & 3.3 & $>64$ \\
\hline$R-148$ & B & $\mathrm{NHCH}_{2}$ & & $3-\mathrm{OCF}_{3}$ & 2.26 & & 6.9 & $<0.13$ & 2.0 & $>64$ \\
\hline$R-149$ & B & $\mathrm{NHCH}_{2}$ & & $2-\mathrm{OCF}_{3}$ & 2.26 & & 8.6 & 0.15 & 2.1 & $>64$ \\
\hline$S-150^{c}$ & A & $\mathrm{NHCO}$ & & $4-\mathrm{OCF}_{3}$ & 1.75 & & 6.0 & 19 & $>64$ & $>64$ \\
\hline$R-150$ & B & $\mathrm{NHCO}$ & & $4-\mathrm{OCF}_{3}$ & 1.75 & & 57 & 2.5 & $>64$ & $>64$ \\
\hline$S-151^{i}$ & A & $\mathrm{NHCO}$ & & $3-\mathrm{OCF}_{3}$ & 1.22 & 0.25 & 5.6 & 25 & $>64$ & $>64$ \\
\hline$R-151$ & B & $\mathrm{NHCO}$ & & $3-\mathrm{OCF}_{3}$ & 1.22 & & 12 & 0.87 & $>64$ & $>64$ \\
\hline$S-152^{i}$ & A & $\mathrm{NHCO}$ & & $2-\mathrm{OCF}_{3}$ & 1.40 & & 5.8 & 53 & $>64$ & $>64$ \\
\hline$R-152$ & B & NHCO & & $2-\mathrm{OCF}_{3}$ & 1.40 & & 18 & 0.96 & $>64$ & $>64$ \\
\hline$S-154^{c}$ & A & $\mathrm{NHCONH}$ & & $4-\mathrm{OCF}_{3}$ & 1.47 & & 10 & 7.5 & 22 & $>64$ \\
\hline$R-154$ & B & NHCONH & & $4-\mathrm{OCF}_{3}$ & 1.47 & & $>64$ & 2.3 & 19 & $>64$ \\
\hline$S-155$ & A & NHCONH & & $2-\mathrm{OCF}_{3}$ & 1.73 & 0.23 & 6.8 & 3.1 & 4.9 & 16 \\
\hline$R-155$ & $\mathrm{~B}$ & NHCONH & & $2-\mathrm{OCF}_{3}$ & 1.73 & & 55 & 1.3 & $>64$ & 48 \\
\hline
\end{tabular}

${ }^{a} \mathrm{IC}_{50}$ values for inhibition of the growth of $L$. don and $L$. inf (in mouse macrophages), T. cruzi (on MRC-5 cells), and T. brucei, or for cytotoxicity toward human lung fibroblasts (MRC-5 cells). ${ }^{b}$ Each value (except the single-test $L$. don data) is the mean of at least two independent determinations. For complete results (mean \pm SD), see the Supporting Information. ${ }^{c}$ From ref $32 .{ }^{d}$ From ref $38 .{ }^{e}$ From ref $40 .{ }^{f}$ LMPH data (mean of three values). ${ }^{g}$ Racemic nitrotriazolooxazine analogue of $R-136 .{ }^{h}$ From ref $30 .{ }^{i}$ From ref $48 .{ }^{j} N$-Piperazine.

best $\left(R-119, L\right.$. inf $\mathrm{IC}_{50}$ of $\left.1.4 \mu \mathrm{M}\right)$, followed by meta substitution ( $R$-118), mimicking findings for biphenyl linkage. Switching to a propargyl ether ${ }^{42}(R-122)$ produced much greater activity ( $L$. inf $\mathrm{IC}_{50}$ of $\left.0.33 \mu \mathrm{M}\right)$, which was largely retained in trifluoromethylpyridine replacements for the aryl ring $^{25}$ (L. inf $\mathrm{IC}_{50}$ s of 0.44 and $0.53 \mu \mathrm{M}$ for $R-123$ and $R-124$, respectively). Conversely, removal of the benzylic methylene
(R-6) also enabled high potency ( $L$. inf $\mathrm{IC}_{50}$ of $0.53 \mu \mathrm{M}, L$. don $\mathrm{IC}_{50}$ of $\left.0.19 \mu \mathrm{M}\right)$, while critically allowing the retention of good aqueous solubility $(12 \mu \mathrm{g} / \mathrm{mL})$ and high microsomal stability [79-81\% parent after $1 \mathrm{~h}$ in MLM and HLM (Table 5)]. Therefore, we similarly investigated trifluoromethylpyridine analogues of $R-6$ ( $R-136, R-137$, and $R-138)$ and while there was an $\sim 2$-fold loss of activity, $R$-136 demonstrated a 9-fold 
Table 4. In Vitro Antiparasitic Activities of 6-O-Linked Biaryl Nitroimidazooxazines
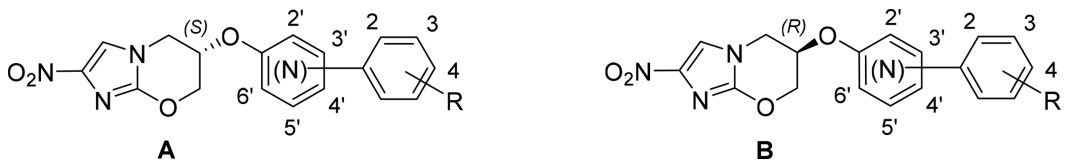

\begin{tabular}{|c|c|c|c|c|c|c|c|c|c|c|}
\hline \multirow[b]{2}{*}{ compd } & \multirow[b]{2}{*}{ form } & \multirow[b]{2}{*}{ link } & \multirow[b]{2}{*}{ aza } & \multirow[b]{2}{*}{$\mathrm{R}$} & \multirow[b]{2}{*}{ CLogP } & \multicolumn{5}{|c|}{$\mathrm{IC}_{50}{ }^{a, b}(\mu \mathrm{M})$} \\
\hline & & & & & & L. don & L. inf & T. cruzi & T. brucei & MRC-5 \\
\hline$R-157$ & B & $3^{\prime}$ & $2^{\prime}$ & $4-\mathrm{OCF}_{3}$ & 3.27 & & 0.31 & $<0.13$ & 7.5 & $>64$ \\
\hline$R-158$ & B & $3^{\prime}$ & $2^{\prime}$ & $4-F$ & 2.35 & & 0.41 & 0.30 & $>64$ & $>64$ \\
\hline$R-160$ & B & $4^{\prime}$ & $2^{\prime}$ & $4-\mathrm{OCF}_{3}$ & 3.35 & & 0.64 & 0.11 & 9.3 & 42 \\
\hline$R-161$ & B & $4^{\prime}$ & $2^{\prime}$ & $4-F$ & 2.43 & & 0.43 & $<0.13$ & 10 & $>64$ \\
\hline$R-168$ & $\mathrm{~B}$ & $4^{\prime}$ & $3^{\prime}$ & $4-\mathrm{OCF}_{3}$ & 3.07 & & 0.13 & 0.15 & $>64$ & $>64$ \\
\hline$R-169$ & B & $4^{\prime}$ & $3^{\prime}$ & $4-F$ & 2.15 & & 0.20 & 0.40 & $>64$ & $>64$ \\
\hline$S-171^{c}$ & A & $3^{\prime}$ & $4^{\prime}, 6^{\prime}$ & $4-\mathrm{OCF}_{3}$ & 2.79 & 0.10 & 23 & 2.0 & $>64$ & 47 \\
\hline$R-171$ & B & $3^{\prime}$ & $4^{\prime}, 6^{\prime}$ & $4-\mathrm{OCF}_{3}$ & 2.79 & & 21 & $<0.13$ & $>64$ & $>64$ \\
\hline$R-172$ & B & $3^{\prime}$ & $4^{\prime}, 6^{\prime}$ & $4-\mathrm{F}$ & 1.87 & & 49 & 0.68 & $>64$ & $>64$ \\
\hline$R-174$ & B & $4^{\prime}$ & $2^{\prime}, 6^{\prime}$ & $4-\mathrm{OCF}_{3}$ & 2.71 & & 0.65 & 0.48 & $>64$ & $>64$ \\
\hline$R-175$ & B & $4^{\prime}$ & $2^{\prime}, 6^{\prime}$ & 4-F & 1.80 & & 0.83 & 0.64 & $>64$ & $>64$ \\
\hline
\end{tabular}

${ }^{a} \mathrm{IC}_{50}$ values for inhibition of the growth of $L$. don and $L$. inf (in mouse macrophages), T. cruzi (on MRC-5 cells), and T. brucei, or for cytotoxicity toward human lung fibroblasts (MRC-5 cells). ${ }^{b}$ Each value (except the single-test L. don data) is the mean of at least two independent determinations. For complete results (mean $\pm \mathrm{SD}$ ), see the Supporting Information. ${ }^{c}$ From ref 42.

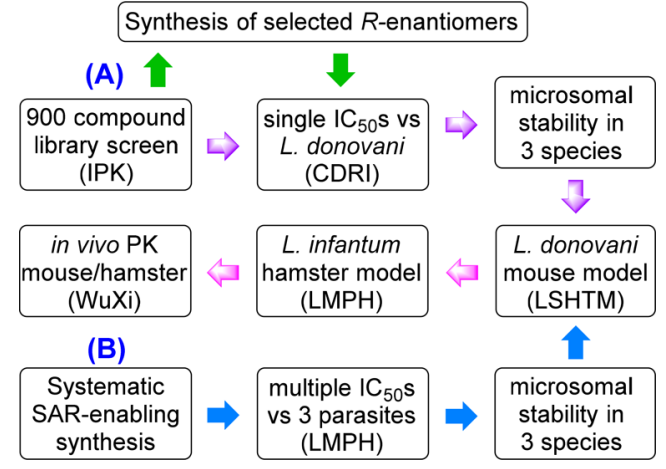

Figure 2. Schematic diagram of the two lead optimization approaches (A and B) employed.

improvement in aqueous solubility $(110 \mu \mathrm{g} / \mathrm{mL})$, together with a slower rate of metabolism (90-92\% parent after $1 \mathrm{~h}$ in MLM and HLM). However, changing to an O-carbamate linker ${ }^{48}$ ( $R$ $140, R-141$, and $R-143$ ) proved to be less satisfactory, with moderate to low potencies observed ( $L$. inf $\mathrm{IC}_{50} \mathrm{~s}$ of $2.1-17$ $\mu \mathrm{M})$.

Another option to improve solubility was to replace the ether linkage at C-6 with nitrogen-based linkers. ${ }^{48}$ The 6-amino analogue of $R-1(R-147)$ had a 5-fold better solubility value at $\mathrm{pH} 7(84 \mu \mathrm{g} / \mathrm{mL})$ and was $>2000$ times more soluble at $\mathrm{pH} 1$. Nevertheless, this compound was less stable toward microsomes (e.g., $61 \%$ vs $86 \%$ in MLM) and showed 2.6-fold lower activity ( $L$. inf $\mathrm{IC}_{50}$ of 12 vs $4.7 \mu \mathrm{M}$ ), which was not sufficiently improved by varying the ring substituent position $(R-148$ and $R$-149). Alternatively, with a carboxamide or urea linker, the enantiomer preference was reversed, with the original $6 S$ forms (S-150, $S$-151, S-152, S-154, and $S$-155) being clearly superior but not particularly potent $\left(L\right.$. inf $\mathrm{IC}_{50} \mathrm{~s}$ of $\left.\sim 6-10 \mu \mathrm{M}\right)$. Hence, the only compounds with useful antileishmanial activity were ether-linked at C-6, although it was apparent that the original $\mathrm{OCH}_{2}$ linkage was not optimal and that removal of the benzylic methylene may have metabolic stability and potency advantages. To investigate this further, a small set of O-linked phenylpyridine and phenylpyrimidine derivatives was evaluated
(Table 4). In the phenylpyridine series ( $R-157$ to $R-169)$, activity against $L$. inf was similar to or better than that for $R-6$, with the 3-pyridine isomers preferred $\left(R-168\right.$ and $R-169, I_{50} \mathrm{~S}$ of $0.13-0.20 \mu \mathrm{M})$. These latter compounds exhibited microsomal stabilities comparable to those of the parent linker series above, but their solubility values were inferior $(0.36-1.2 \mu \mathrm{g} /$ $\mathrm{mL}$ at $\mathrm{pH} 7)$. Finally, contrary to the screening data for $S-\mathbf{1 7 1}$, a proximal pyrimidine ring was tolerated only when it was paralinked (R-174 and R-175, L. inf $\mathrm{IC}_{50} \mathrm{~s}$ of $\left.0.65-0.83 \mu \mathrm{M}\right)$. Generally, potency against $L$. inf was more discriminating and tended to better correlate with in vivo outcomes. ${ }^{24,25}$

Throughout the course of these studies, 10 more candidates (R-1, R-6, R-69, R-96, R-99, R-106, R-136, R-147, R-168, and $R-169)$ were screened for activity in the mouse VL model at 50 $\mathrm{mg} / \mathrm{kg}$ [dosing po daily for $5 \mathrm{~d}$ (Table 5)]. The ortho-linked phenylpyridine $R-69$ was disappointing (20\% inhibition), but the new para-linked analogues $R-96, R-99$, and $R-106$ displayed high efficacies (91-97\%). More heartening still was the fact that shorter chain phenylpyridines $R-\mathbf{1 6 8}$ and $R-169$ gave essentially complete clearance of the parasite infection (99.9\%), as did their monoaryl counterparts, R-6 and R-136. However, both $R-1$ and its amino-linked equivalent $R-147$ were unsatisfactory (50 and 12\%, respectively), consistent with their weaker in vitro potencies. During the concluding stages of this project, Patterson et al. ${ }^{38}$ reported that $R-\mathbf{1}$ was a potential oral treatment for $\mathrm{VL}$ on the basis of its in vivo activity in a comparable mouse model at a much larger dose of $100 \mathrm{mg} / \mathrm{kg}$ twice daily, but it is clear from these results and other studies ${ }^{24}$ that $R-1$ may not be the optimal development candidate.

Additional Assessments To Determine the Best VL Lead. Dose-response experiments on 7 of the 10 best compounds (R-6, R-84, R-89, R-91, R-92, R-96, and R-99) in the mouse VL model yielded $\mathrm{ED}_{50}$ values of $7.5,12,14,28,20$, 28 , and $19 \mathrm{mg} / \mathrm{kg}$, respectively (Table 5 and Figure 4). The more recent O-linked leads, $R-136, R-168$, and $R-169$, were also evaluated at a smaller dose of $6.25 \mathrm{mg} / \mathrm{kg}$ and provided parasite burden reductions of $30,99.7$, and $72 \%$, respectively. The most efficacious of these $(R-168)$ produced $84 \%$ inhibition at $3.13 \mathrm{mg} / \mathrm{kg}$, representing activity at a level similar to that of 
Table 5. Aqueous Solubility, Microsomal Stability, and in Vivo (mouse) Antileishmanial Efficacy Data for Selected Analogues

\begin{tabular}{|c|c|c|c|c|c|c|c|c|c|c|}
\hline \multirow[b]{2}{*}{ compd } & \multicolumn{2}{|c|}{ aqueous solubility $^{a}(\mu \mathrm{g} / \mathrm{mL})$} & \multicolumn{3}{|c|}{ microsomal stability ${ }^{b}$ [\% remaining at $1(0.5) \mathrm{h}$ ] } & \multicolumn{5}{|c|}{ in vivo efficacy against $L$. don (\% inhibition at dose in $\mathrm{mg} / \mathrm{kg}$ ) } \\
\hline & $\mathrm{pH} 7$ & $\mathrm{pH} 1$ & $\mathrm{H}$ & $\mathrm{M}$ & Ham & 50 & 25 & 12.5 & 6.25 & $\mathrm{ED}_{50}{ }^{d}$ \\
\hline S-1 & 19 & & 82 & 94 & & & & & & \\
\hline$R-1$ & 18 & & 92 & 86 & 31 & 50 & & & & \\
\hline$R-6$ & 12 & & 81 & 79 & 19 & $>99$ & $>99$ & 81 & 42 & 7.5 \\
\hline 22 & 6.0 & & 92 & 72 & & & & & & \\
\hline 24 & 3.9 & & (78) & (88) & (70) & & 31 & & & \\
\hline$S-51$ & 1.6 & & (78) & $(75)$ & $(43)$ & & & & & \\
\hline$S-52$ & 2.7 & & 87 & 67 & & & & & & \\
\hline$R-69$ & 0.51 & 263 & 35 & 44 & 3.0 & 20 & & & & \\
\hline$R-74$ & 78 & 7350 & 8.0 & 0.2 & 0.1 & & & & & \\
\hline S-77 & 3.0 & 211 & (75) & (78) & (31) & 44 & & & & \\
\hline R-77 & 1.5 & 167 & (68) & (68) & $(31)$ & 52 & & & & \\
\hline$S-81$ & 15 & 439 & (96) & (74) & (15) & 35 & & & & \\
\hline$R-81$ & 6.0 & 691 & (79) & (68) & (0) & & & & & \\
\hline$R-84$ & 3.0 & 1040 & $27(66)$ & $36(70)$ & $10(61)$ & $>99$ & 76 & 42 & 36 & 12 \\
\hline$S-89$ & 1.4 & 479 & (88) & (84) & (69) & 45 & & & & \\
\hline$R-89$ & 3.4 & 503 & $27(75)$ & $40(79)$ & $10(69)$ & $>99$ & 72 & 48 & 17 & 14 \\
\hline$S-91$ & 1.4 & 384 & (73) & $(100)$ & $(75)$ & 69 & & & & \\
\hline$R-91$ & 2.9 & 12 & $16(65)$ & $27(81)$ & $5.8(57)$ & $>99$ & 38 & 6 & & 28 \\
\hline$S-92$ & 4.0 & 1780 & (92) & (88) & (59) & 37 & & & & \\
\hline$R-92$ & 5.7 & 2050 & $49(71)$ & $56(86)$ & $11(45)$ & 94 & 54 & 20 & & 20 \\
\hline$R-94$ & 11 & 4600 & $58(85)$ & $66(77)$ & $10(37)$ & 83 & & & & \\
\hline$R-96$ & 40 & 7140 & 59 & 57 & 5.2 & 97 & 48 & 29 & 17 & 28 \\
\hline$R-99$ & 2.9 & 364 & 37 & 41 & 9.2 & 97 & 64 & 31 & 8 & 19 \\
\hline$R-102$ & 2.1 & 857 & 58 & 56 & 7.9 & & & & & \\
\hline$R-106$ & 3.9 & 925 & 58 & 67 & 10 & 91 & & & & \\
\hline 116 & 0.13 & 32 & (88) & (96) & (88) & & 49 & & & \\
\hline 117 & 0.27 & 132 & (85) & (66) & (64) & & 23 & & & \\
\hline$R-136$ & 110 & & 90 & 92 & 48 & $>99$ & & & 30 & \\
\hline$R-147$ & 84 & 38100 & 84 & 61 & 7.5 & 12 & & & & \\
\hline$S-151$ & 132 & & (100) & (83) & (81) & 72 & & & & \\
\hline$R-151$ & 85 & & 87 & 86 & 59 & 5 & & & & \\
\hline$S-155$ & 22 & & (74) & (43) & (64) & & & & & \\
\hline$R-168$ & 0.36 & 36 & 40 & 35 & & $>99$ & & & $>99$ & $<3.1$ \\
\hline$R-169$ & 1.2 & 325 & 73 & 59 & & $>99$ & & & 72 & \\
\hline
\end{tabular}

${ }^{a}$ Kinetic solubility in water $(\mathrm{pH} 7)$ or $0.1 \mathrm{M} \mathrm{HCl}(\mathrm{pH} 1)$ at $20^{\circ} \mathrm{C}$, determined by HPLC (see Method A in Experimental Section). ${ }^{b}$ Pooled human $(\mathrm{H})$, CD-1 mouse $(\mathrm{M})$, or hamster (Ham) liver microsomes; data in parentheses are the percentage parent compound remaining following a 30 min incubation. ${ }^{c}$ Dosing was done orally, once daily for 5 days consecutively; data are the mean percentage reduction of parasite burden in the liver. ${ }^{d}$ Dose in milligrams per kilogram required to achieve a mean $50 \%$ reduction in parasite burden.

the nitroimidazooxazole $4,^{25,26}$ albeit marginal aqueous solubility $(0.85 \mu \mathrm{M}$ at $\mathrm{pH} 7)$ deterred its advanced assessment. Instead, we elected to focus initially on the monoaryl ethers $R-6$ and $R-136$, together with phenylpyridines $R-84$ and $R-89$, which all produced favorable mouse PK data, including excellent oral exposure levels and half-lives of 6-30 h (Table 6 and Figure S1).

The selected candidates were further assessed in the chronic infection hamster model, which is considered the bona fide experimental model for VL because it mimics many features of progressive human disease. ${ }^{51}$ Pleasingly, at $50 \mathrm{mg} / \mathrm{kg}$ twice daily for 5 days, phenylpyridine $R-84$ achieved $99.9-100 \% L$. inf clearance in all three target organs, and R-89 was almost as good (Table 7 and Figure 5), although both compounds were less inhibitory in bone marrow at $25 \mathrm{mg} / \mathrm{kg}$ b.i.d. (81-88\%). The monoaryl ethers $R-\mathbf{6}$ and $R-\mathbf{1 3 6}$ were even more effective at both dose levels, enabling a $97-99 \%$ parasite kill at $25 \mathrm{mg} / \mathrm{kg}$ b.i.d. (for comparison, this efficacy level was similar to that observed for 3 at $12.5 \mathrm{mg} / \mathrm{kg}$ b.i.d. ${ }^{24}$ ). Pyridinyl ether $R-136$ additionally demonstrated fully curative activity (100\% parasite clearance in all three organs) at $25 \mathrm{mg} / \mathrm{kg}$ b.i.d. in an $L$. don infection hamster model. Three more phenylpyridines ( $R-96$, $R-99$, and $R-106$ ) were similarly evaluated in the $L$. inf hamster model, but only $R-99$ (the 3-pyridyl isomer of $R-89$ ) showed any promise $(86-98 \%$ at $50 \mathrm{mg} / \mathrm{kg}$ b.i.d.), paralleling efficacy trends in the mouse model. These results were also broadly in line with the hamster PK data, where R-96 and R-106 displayed inferior oral exposures and oral bioavailabilities (21-37\%) much lower than those of the other leads [64-100\% (Table 6 and Figure S2)].

To better discriminate among the four preferred candidates, we next considered key safety features, starting with measuring their interactions with the hERG channel. While the monoaryl ethers $R-6$ and $R-136$ posed minimal risk (hERG $\mathrm{IC}_{50} \mathrm{~s}$ of $>30$ $\mu \mathrm{M})$, unfortunately, phenylpyridines $R-84$ and $R-89$ both caused potent inhibition $\left(\mathrm{IC}_{50} \mathrm{~s}\right.$ of 0.81 and $0.92 \mu \mathrm{M}$, respectively), indicating a strong likelihood of QT prolongation $^{52}$ (lead optimization criteria ${ }^{53,54}$ mandate an $\mathrm{IC}_{50}$ of $>10$ $\mu \mathrm{M})$. This outcome was not anticipated, as $6 \mathrm{~S}$ counterparts had generated much less concern. The two remaining compounds 
Table 6. Pharmacokinetic Parameters for Selected Compounds in Mice, Rats, and Hamsters

\begin{tabular}{|c|c|c|c|c|c|c|c|c|c|c|}
\hline \multirow[b]{2}{*}{ compd } & \multicolumn{5}{|c|}{ intravenous $(1-2 \mathrm{mg} / \mathrm{kg})^{a}$} & \multicolumn{5}{|c|}{ oral $(25-50 \mathrm{mg} / \mathrm{kg})^{a}$} \\
\hline & $C_{0}(\mu \mathrm{g} / \mathrm{mL})$ & $\mathrm{CL}(\mathrm{mL} / \mathrm{min} / \mathrm{kg})$ & Vdss (L/kg) & $t_{1 / 2}(\mathrm{~h})$ & $\mathrm{AUC}_{\text {last }}{ }^{b}(\mu \mathrm{g} \cdot \mathrm{h} / \mathrm{mL})$ & $C_{\max }(\mu \mathrm{g} / \mathrm{mL})$ & $T_{\max }(\mathrm{h})$ & $t_{1 / 2}(\mathrm{~h})$ & $\mathrm{AUC}_{\text {last }}{ }^{b}(\mu \mathrm{g} \cdot \mathrm{h} / \mathrm{mL})$ & $F^{c}(\%)$ \\
\hline \multicolumn{11}{|c|}{ Mice } \\
\hline$R-6$ & & & & & & 21 & 5.3 & 7.8 & 272 & \\
\hline 24 & 0.34 & 13 & 2.5 & 2.0 & 1.25 & 1.5 & 1.0 & & 15.7 & 50 \\
\hline$R-84$ & & & & & & 16 & 4.7 & 9.4 & 211 & \\
\hline$R-89$ & & & & & & 32 & 3.3 & 6.1 & 265 & \\
\hline 116 & 0.75 & 1.2 & 1.9 & 20 & 11.7 & 0.87 & 10 & & 32.2 & 11 \\
\hline 117 & 0.44 & 5.2 & 1.8 & 2.7 & 3.18 & 0.84 & 8.0 & & 12.0 & 15 \\
\hline$R-136$ & & & & & & 96 & 3.1 & 30 & 1777 & \\
\hline \multicolumn{11}{|c|}{ Rats } \\
\hline$R-6$ & 0.54 & 11 & 2.7 & 3.0 & 1.51 & 5.2 & 6.7 & $-d$ & 80.6 & 100 \\
\hline$R-136$ & 0.84 & 6.1 & 1.5 & 2.7 & 2.47 & 18 & 2.7 & 3.5 & 164 & 100 \\
\hline \multicolumn{11}{|c|}{ Hamsters } \\
\hline$R-6$ & 0.66 & 81 & 6.5 & 1.4 & 0.42 & 2.1 & 3.3 & 2.2 & 11.2 & 100 \\
\hline$R-84$ & 0.65 & 23 & 8.2 & 6.3 & 1.30 & 5.7 & 3.0 & 5.8 & 62.7 & 100 \\
\hline$R-89$ & 0.94 & 18 & 4.2 & 3.9 & 1.89 & 4.2 & 2.0 & 3.9 & 26.7 & 74 \\
\hline$R-96$ & 0.93 & 63 & 4.0 & 0.83 & 0.48 & 1.9 & 0.75 & 0.83 & 3.95 & 37 \\
\hline R-99 & 1.1 & 8.8 & 3.2 & 5.3 & 3.69 & 4.7 & 3.3 & 5.8 & 46.5 & 64 \\
\hline$R-106$ & 0.52 & 59 & 5.4 & 1.4 & 0.71 & 1.1 & 1.7 & 17 & 3.40 & 21 \\
\hline$R-136$ & & & & & & 12 & 3.3 & 3.1 & 71.0 & \\
\hline
\end{tabular}

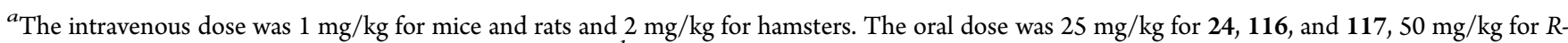
106 and $R-136$, and $40 \mathrm{mg} / \mathrm{kg}$ for the other compounds. ${ }^{b}$ Area under the curve calculated to the last time point $(24$ or $48 \mathrm{~h}) .{ }^{c}$ Oral bioavailability, determined using dose-normalized $\mathrm{AUC}_{\text {last }}$ values. ${ }^{d}$ Not calculable.<smiles>O=[N+]([O-])c1cn2c(n1)OC[C@@H](OCc1csc(-c3ccc(OC(F)(F)F)cc3)n1)C2</smiles><smiles>COc1ccc(-n2ccc(COC3COc4nc([N+](=O)[O-])cn4C3)n2)cc1</smiles><smiles>O=[N+]([O-])c1cn2c(n1)OCC(OCc1ccc(Oc3ccc(OC(F)(F)F)cc3)cc1)C2</smiles><smiles>COc1cc(-c2ccc(OC(F)(F)F)cc2)ccc1CO[C@H]1COc2nc([N+](=O)[O-])cn2C1</smiles><smiles>CS(=O)(=O)c1cn2c(n1)OC[C@H](OCc1cccc(-c3ccccc3OC(F)(F)F)c1)C2</smiles>
(95\%@ $10 \mu \mathrm{g} / \mathrm{mL})$ IC $_{50} 0.36 \mu \mathrm{M}$<smiles>C/C=C/[N+](=O)[O-]</smiles><smiles>FC(F)(F)Oc1ccc(-c2ccccc2CO[C@H]2COc3ncnn3C2)cc1</smiles>

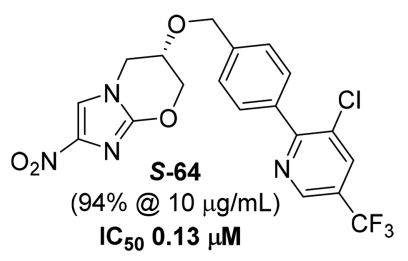<smiles>O=[N+]([O-])c1cn2c(n1)OC[C@H](OCc1cccc(-c3ccc(OC(F)(F)F)cc3)n1)C2</smiles>

$\mathrm{IC}_{50} 0.12 \mu \mathrm{M}$ $\mathrm{IC}_{50} 0.24 \mu \mathrm{M}$<smiles>CCCCOc1ccc(-c2ccc(CO[C@H]3COc4nc([N+](=O)[O-])cn4C3)cn2)c(Cl)c1</smiles><smiles>CCCOc1ccc(-c2ccc(CO[C@H]3COc4nc([N+](=O)[O-])cn4C3)nc2)cc1</smiles><smiles>O=[N+]([O-])c1cn2c(n1)OC[C@H](OCc1ccc(-c3ccc(OC(F)(F)F)cc3F)cn1)C2</smiles>

$\mathrm{IC}_{50} 0.08 \mu \mathrm{M}$

IC $_{50} 0.18 \mu \mathrm{M}$ 

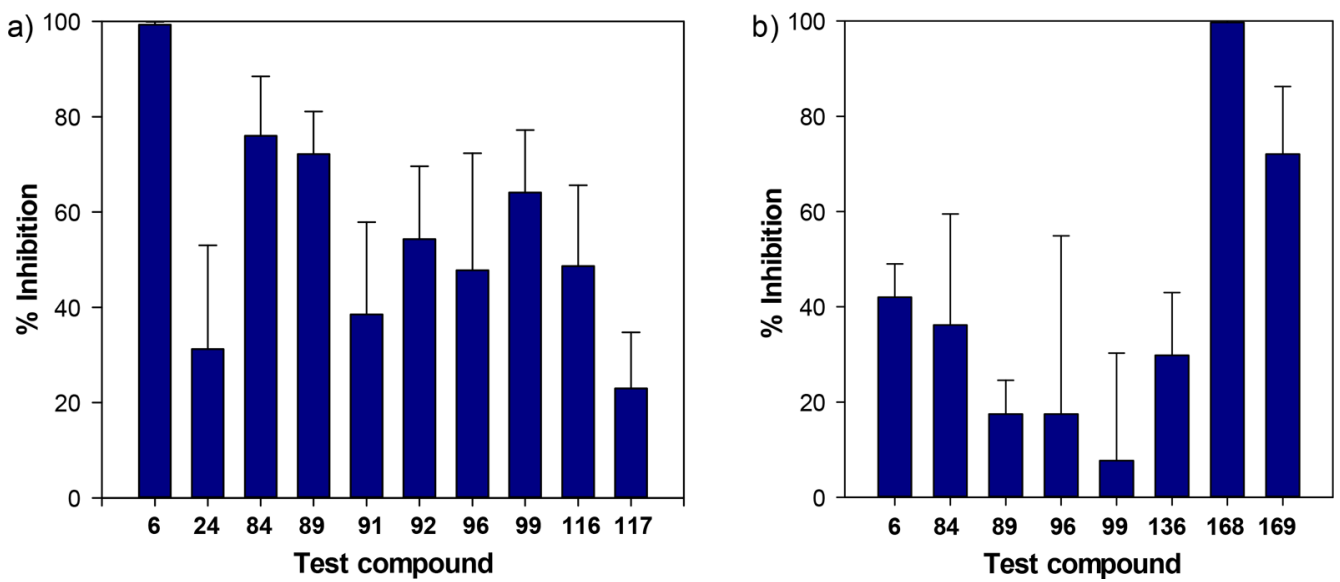

Figure 4. Comparative in vivo efficacy in the $L$. don mouse model: (a) $25 \mathrm{mg} / \mathrm{kg}$ and (b) $6.25 \mathrm{mg} / \mathrm{kg}$. All compounds except racemates 24 , 116, and 117 are the $6 R$ form.

Table 7. In Vivo Efficacy Data for Selected Compounds in the Early Curative L. inf Hamster Model

\begin{tabular}{lcccc} 
& & \multicolumn{3}{c}{ \% inhibition in target organs ${ }^{b}$} \\
\cline { 3 - 5 } compd & dose $^{a}(\mathrm{mg} / \mathrm{kg})$ & liver & spleen & bone marrow \\
MIL & 40 & 99.0 & 99.5 & 96.8 \\
$R-6$ & 50 & 100 & 100 & 99.8 \\
& 25 & 98.4 & 99.2 & 97.0 \\
$R-84$ & 12.5 & 53.5 & 47.6 & 37.3 \\
& 50 & 100 & 99.9 & 99.9 \\
$R-89$ & 25 & 98.1 & 98.4 & 88.3 \\
& 12.5 & 69.6 & 55.7 & 33.6 \\
$R-96$ & 50 & 99.9 & 99.9 & 98.9 \\
$R-99$ & 25 & 99.1 & 93.7 & 81.3 \\
$R-106$ & 12.5 & 83.5 & 70.6 & 50.4 \\
$R-136$ & 50 & 73.0 & 55.1 & 55.7 \\
& 50 & 97.7 & 97.5 & 86.0 \\
& 50 & 55.7 & 17.6 & 45.0 \\
& 50 & 100 & 100 & 99.9 \\
& 25 & 99.5 & 97.3 & 97.7 \\
& 12.5 & 44.4 & 43.0 & 53.0 \\
\hline 11 & 50 & &
\end{tabular}

${ }^{a}$ All test compounds were dosed orally, twice daily for 5 days consecutively; miltefosine (MIL) was dosed once daily for the same period. ${ }^{b}$ Data are the mean percentage reductions in parasite burden in target organs. were then checked for any evidence of mutagenicity in the Ames test. Here, phenyl ether R-6 was negative, but the more soluble pyridinyl ether $R-136$ unexpectedly yielded a positive result. Although several other nitroimidazole drugs are Ames positive (e.g., metronidazole and fexinidazole), ${ }^{55}$ this outcome effectively ruled $R-\mathbf{1 3 6}$ out of contention because it would face a more difficult path to achieving regulatory approval. ${ }^{16,53}$ Thus, $R-6$ was identified as the optimal VL lead.

Further Appraisal of VL lead R-6. Additional properties of $R-6$ were measured and weighed against those of the initial preclinical candidate 4 (Table 8 ). The two compounds were comparable in terms of molecular weight (345 Da vs $359 \mathrm{Da}$ ) and were both highly permeable, but $R-6$ had a lower measured $\log \mathrm{D}$ value ( 2.59 vs 3.10 for 4 and 2.52 for $S-\mathbf{1}^{48}$ ), superior thermodynamic solubility $(23 \mu \mathrm{M}$ vs $2.8 \mu \mathrm{M})$, and a reduced propensity to bind to plasma proteins in various species (82$87 \%$ vs $92-96 \%$ for 4 ). This lead also showed only weak CYP3A4 activity $\left(\mathrm{IC}_{50}>40 \mu \mathrm{M}\right)$ and produced a notably favorable rat PK profile, with prolonged exposure and $100 \%$ oral bioavailability (Table 6 and Figure S2). These attributes reinforced our conclusion that R-6 (DNDI-8219) was indeed a very promising backup candidate for $\mathrm{VL}$.

A larger scale synthesis of $R-6$ has recently provided a single $170 \mathrm{~g}$ batch of high-quality material (HPLC purity of $>99.9 \%$ and $97.2 \%$ ee) in reasonable overall yield ( $8 \%$ over nine linear

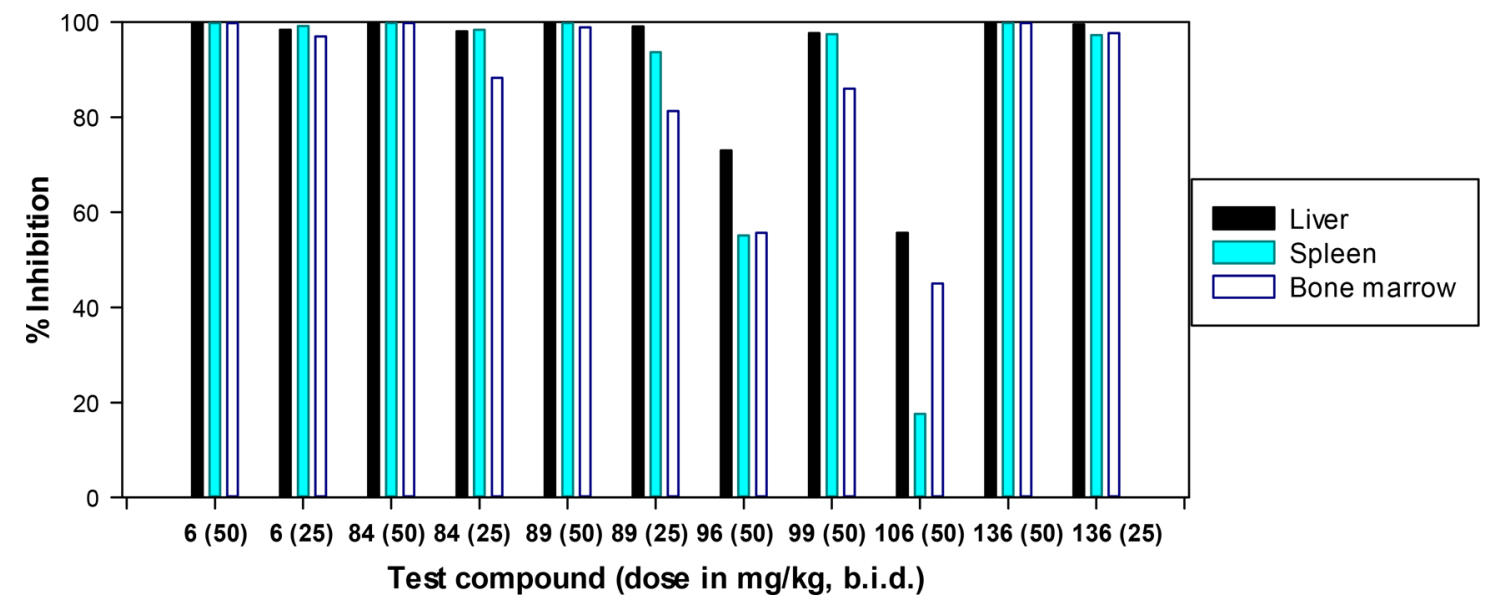

Figure 5. Comparative in vivo efficacy in the L. inf hamster model. All compounds are the $6 R$ form. 
Table 8. Additional Comparative Data for Lead Compounds 4 and $R-6$

\begin{tabular}{|c|c|c|}
\hline property & $4^{a}$ & $R-6$ \\
\hline molecular weight (Da) & 359.3 & 345.2 \\
\hline LogD (measured) & 3.10 & 2.59 \\
\hline thermodynamic solubility $(\mu \mathrm{M})$ at $\mathrm{pH} 7.4$ & 2.8 & 23 \\
\hline $\begin{array}{l}\text { permeability, Papp }\left(\times 10^{-6} \mathrm{~cm} / \mathrm{s}\right) \mathrm{A} \text { to } \mathrm{B} / \mathrm{B} \text { to } \mathrm{A} \\
\text { plasma protein binding }(\%)\end{array}$ & $22.6 / 24.7^{b}$ & $32.4 / 18.9^{c}$ \\
\hline mouse & 96.2 & 86.7 \\
\hline rat & 93.2 & 82.1 \\
\hline hamster & 92.4 & 87.2 \\
\hline human & 93.9 & 85.2 \\
\hline mutagenic effect (Ames test) & no & no ${ }^{d}$ \\
\hline $\mathrm{hERG} \mathrm{IC}_{50}(\mu \mathrm{M})$ & 10.5 & $>30$ \\
\hline CYP3A4 $\mathrm{IC}_{50}(\mu \mathrm{M})$ & $>25$ & $>40$ \\
\hline MABA MIC $(\mu \mathrm{M})$ & 0.046 & $31^{e}$ \\
\hline LORA MIC $(\mu \mathrm{M})$ & 5.9 & $>128$ \\
\hline
\end{tabular}

${ }^{a}$ Most data from refs 24 and $25 .{ }^{b} \mathrm{Caco}-2$ data from ref $61 .{ }^{c} \mathrm{MDCK}-$ MDR1 data; no P-gp-mediated efflux. ${ }^{d}$ Not mutagenic in strains TA98 and TA100, in the presence or absence of metabolic activation (S9 fraction). ${ }^{e}$ Single MIC against $M$. $t b$, determined under aerobic conditions.

steps, starting from commercial $R$-solketal). However, the current synthetic route would still require significant improvement to deliver a scalable, robust, and cost-effective chemical process, in line with the stated TPP objectives for an affordable drug. Possible alternatives include using the known ${ }^{56}$ enantiomer of orthogonally diprotected triol 125 and following the pathway described for S-6 in Scheme 4B because this enantiomer could be obtained from the cheap, optically pure starting material D-mannitol (via the less expensive $S$ solketal). ${ }^{57}$

Mechanistic studies of the closely related nitroimidazooxazine $R-1$ demonstrated that it was not activated by the previously identified type I nitroreductase (NTR1) in Leishmania, which mediates the cidal effects of monocyclic nitroheterocyclic drugs such as fexinidazole and nifurtimox. ${ }^{38,58}$ Instead, the activity of $R-1$ was solely triggered by the same novel flavin mononucleotide-dependent $\mathrm{NADH}$ oxidoreductase (NTR2) in Leishmania that was employed by nitroimidazooxazoles, such as 4 and $5 .{ }^{27}$ This new mode of action was elucidated through a combination of quantitative proteomics and whole genome sequencing of susceptible and drug resistant L. don promastigotes, the latter being generated via culture in the continuous presence of $R-1$ for 80 days (leading to a reduced level of expression of NTR2). ${ }^{27}$ The further observation that $R-\mathbf{1}$ and fexinidazole sulfone displayed additive effects against drug susceptible $L$. don sparked the suggestion of combination therapy between monocyclic and bicyclic nitro drugs to reduce the likelihood of any future clinical drug resistance. ${ }^{38}$ However, we consider that it may be more preferable to look for alternative partner drugs for 3 (or R-6) with greater diversity in their mechanism of action.

One final aspect to consider with $R-6$ was its efficacy against a wider range of $\mathrm{VL}$ and cutaneous leishmaniasis (CL) strains. Overall, R-6 displayed potent broad-spectrum activity against both reference strains and clinical isolates (Table 9), comparing favorably with the standard agents sodium stibogluconate, paromomycin, and miltefosine. This lead was also effective against the drug resistant clinical isolates $L$. don BHU1, L. inf LEM5159, and L. inf MHOM/FR/96/LEM3323 ( IC $_{50}$ s of 1.3-
Table 9. Inhibitory Activity of R-6 and Clinical VL Drugs against Different Leishmania Strains

\begin{tabular}{|c|c|c|c|c|c|}
\hline \multirow[b]{2}{*}{ strain (origin) } & \multicolumn{5}{|c|}{$\mathrm{IC}_{50}(\mu \mathrm{M})^{a}$} \\
\hline & $R-6$ & $\mathrm{SSG}^{b}$ & $A m p B^{c}$ & $\mathrm{MIL}^{d}$ & $\mathrm{PM}^{e}$ \\
\hline \multicolumn{6}{|c|}{ VL Strains } \\
\hline $\begin{array}{l}\text { L. don } \mathrm{MHOM} / \mathrm{IN} / 80 / \mathrm{DD} 8 \\
\text { (India) }\end{array}$ & 0.22 & 54.3 & 0.02 & 2.50 & $>30$ \\
\hline $\begin{array}{l}\text { L. don } \mathrm{MHOM} / \mathrm{ET} / 67 / \mathrm{HU} 3 \\
\text { (Ethiopia) }\end{array}$ & 0.33 & NT & 0.05 & 2.05 & NT \\
\hline $\begin{array}{l}\text { L. inf MHOM/MA/67/ } \\
\text { ITMAP263 (Morocco) }\end{array}$ & 0.51 & NT & NT & 2.30 & 136 \\
\hline $\begin{array}{l}\text { L. don } \mathrm{MHOM} / \mathrm{SD} / 62 / \\
\text { 1SCL2D (Sudan) }\end{array}$ & 2.16 & NT & NT & NT & NT \\
\hline $\begin{array}{l}\text { L. inf MHOM/FR/96/ } \\
\text { LEM3323 C14 MIL4 } \\
\text { (France) }^{f}\end{array}$ & 0.59 & NT & NT & $>20$ & 78.5 \\
\hline \multicolumn{6}{|c|}{ VL Clinical Isolates } \\
\hline L. don BHU1 (India) ${ }^{g}$ & 1.34 & $>150$ & 0.20 & 3.80 & $>30$ \\
\hline L. don SUKA001 (Sudan) & 0.57 & 29.9 & 0.05 & 2.13 & $>30$ \\
\hline L. don GR265 (Ethiopia) & 0.19 & 14.5 & 0.05 & 4.60 & $>30$ \\
\hline L. inf LEM5695 (Algeria; dog) & 1.77 & NT & NT & 1.86 & 165 \\
\hline $\begin{array}{l}\text { L. inf MCAN/BR/2002/BH400 } \\
\text { (Brazil; dog) }\end{array}$ & 1.23 & NT & NT & 1.11 & 64.1 \\
\hline $\begin{array}{l}\text { L. inf L3034 (Paraguay; HIV } \\
\text { patient) }\end{array}$ & 0.75 & NT & NT & 1.25 & 87.7 \\
\hline $\begin{array}{l}\text { L. inf LEM5159 (France; HIV } \\
\text { patient) }\end{array}$ & 5.41 & NT & NT & $>20$ & 64.9 \\
\hline $\begin{array}{l}\text { L. inf LEM3323 (France; HIV } \\
\text { patient) }\end{array}$ & 2.22 & NT & NT & 0.74 & 142 \\
\hline \multicolumn{6}{|c|}{ CL Strains } \\
\hline $\begin{array}{l}\text { Leishmania aethiopica MHOM/ } \\
\text { ET/84/KH (Ethiopia) }\end{array}$ & 3.17 & NT & 0.11 & 36.1 & NT \\
\hline $\begin{array}{l}\text { Leishmania amazonensis } \\
\text { MPRO/BR/72/M1841 } \\
\text { (Brazil) }^{i}\end{array}$ & 4.68 & NT & 0.13 & 15.0 & NT \\
\hline $\begin{array}{l}\text { Leishmania major MHOM/SA/ } \\
\text { 85/JISH118 (Saudi Arabia) }\end{array}$ & 2.34 & NT & 0.05 & 22.3 & NT \\
\hline $\begin{array}{l}\text { Leishmania mexicana MNYC/ } \\
\text { BZ/62/M379 (Belize) }\end{array}$ & 1.17 & NT & 0.08 & 6.55 & NT \\
\hline $\begin{array}{l}\text { Leishmania panamensis } \\
\text { MHOM/PA/67/Boynton } \\
\text { (Panama) }\end{array}$ & 0.34 & NT & 0.07 & 21.3 & NT \\
\hline Leishmania tropica Anwari & 1.57 & $>100$ & 0.11 & 8.22 & NT \\
\hline
\end{tabular}
(Syrian clinical isolate)

${ }^{a}$ NT means not tested; some data for clinical VL drugs from refs 26 and $62 .{ }^{b}$ Sodium stibogluconate ( $\mathrm{IC}_{50}$ in micrograms per milliliter). ${ }^{c}$ Amphotericin B. ${ }^{d}$ Miltefosine. ${ }^{e}$ Paromomycin. ${ }^{f}$ Resistant to miltefosine. ${ }^{g}$ Resistant to sodium stibogluconate. ${ }^{h}$ Failed amphotericin B treatment. ${ }^{i}$ DsRed 2 transgenic strain.

$5.4 \mu \mathrm{M})$, as well as the miltefosine resistant laboratory strain $L$. inf MHOM/FR/96/LEM3323 C14 MIL4 ( $\mathrm{IC}_{50}$ of $\left.0.59 \mu \mathrm{M}\right)$. These data confirm that $R-6$ has excellent potential as a therapy for VL and may have an additional application for the treatment of CL (the more common skin lesion form of leishmaniasis). ${ }^{1}$

SAR of 6-Substituted 2-Nitroimidazo(or 2Nitrotriazolo)oxazines for Chagas Disease. While the primary objective of this reinvestigation of pretomanid analogues was to develop a backup drug candidate for VL, retrospective screening against the protozoan parasites $T$. cruzi and T. brucei presented an opportunity to assess the possible capacity of these compounds to treat Chagas disease and human African trypanosomiasis (HAT), respectively. A brief inspection of Tables $1-4$ found that only one compound (116) had submicromolar activity against HAT (T. brucei $\mathrm{IC}_{50}$ of 0.63 $\mu \mathrm{M})$, and this hit could be disregarded on the basis of its less favorable mouse PK profile, inferior aqueous solubility, and 
poor MDCK-MDR1 cell permeability. ${ }^{36}$ Conversely, like 7substituted 2-nitroimidazooxazines, ${ }^{24}$ the majority of compounds displayed interesting potencies against T. cruzi $\left(\mathrm{IC}_{50} \mathrm{~s}\right.$ of $0.025-1 \mu \mathrm{M})$. The selective anti-Chagas activity of nitrotriazolooxazines (e.g., 49; T. cruzi $\mathrm{IC}_{50}$ of $0.25 \mu \mathrm{M}$ vs $>64 \mu \mathrm{M}$ against $L$. inf) was particularly striking, being reminiscent of that of a nitrotriazolooxazole analogue of $4 .^{25}$ However, further scrutiny revealed that the enantiomers of $\mathbf{4 8}$ were roughly equipotent (Table 3 ), while the racemic nitrotriazole counterparts of key VL leads $R-6, R-84$, and R-136 (47, 88, and 139, respectively) were 9-68-fold less active, suggesting limited utility for this class.

Aside from the less suitable reversed linker congeners (2426,116 , and 117) and O-linked biaryls $(R-157, R-160, R-161$, $R-168$, and $R-171)$, the most potent 6-substituted 2-nitroimidazooxazine anti-Chagas leads were para-linked phenylpyridines (e.g., R-84, R-89, R-90, R-92, and $R-93$; $\mathrm{IC}_{50} \mathrm{~S}$ of $0.025-0.078 \mu \mathrm{M})$. Here, the $6 R$ enantiomers were clearly superior (by 15- to $>400$-fold), and the 2-pyridine isomer was preferred, although $\mathrm{N}$-oxidation of the pyridine ring $(R-85)$ was strongly deactivating (77-fold). In the monoaryl subset, arylpropargyl ether $R-\mathbf{1 2 2}$ and phenyl ether $R-\mathbf{6}$ seemed highly promising ( $\mathrm{IC}_{50} \mathrm{~s}$ of 0.11 and $0.15 \mu \mathrm{M}$, respectively), with the latter favored on PK and solubility grounds, whereas similarly active benzylamines $R-148$ and $R-149$ were expected to show rapid metabolism. ${ }^{36}$ Overall, given the significant hERG liability and higher level of plasma protein binding of the phenylpyridines (e.g., $97.9 \%$ for $R-84$ in mice), $R-6$ was also regarded as the best new lead for Chagas disease, although its mechanism of action for this application remains to be determined.

\section{CONCLUSIONS}

In response to a compelling clinical need for more satisfactory $\mathrm{VL}$ treatments, recent efforts have been made to reposition leads from other therapeutic areas, seeking to accelerate new drug development. Promising results with antitubercular nitroimidazooxazoles and 7-substituted 2-nitroimidazooxazines encouraged us to evaluate additional scaffolds, e.g., nitroimidazooxazepines and methylated or reversed C-6 linker analogues of pretomanid, but these lacked sufficient potency and/or suitable PK and efficacy in the L. don mouse model. However, phenotypic screening of our pretomanid analogue library and follow-up $\mathrm{IC}_{50}$ testing unveiled more active hits spanning a wide lipophilicity range (CLogP values of 1.2-4.5), including several with better solubility and microsomal stability, e.g., phenylpyridines and benzamide S-151. This work also pointed to the generally improved activities of novel $6 R$ enantiomers, which was confirmed for phenylpyridines through comparative appraisal in the mouse VL model. Further studies in this series established that a 4-trifluoromethoxy phenyl substituent, para linkage, and a proximal 2-pyridine ring were preferred for good in vivo PK and efficacy, with two such leads ( $R-84$ and $R-89)$ giving $\geq 99 \%$ parasite clearance in the $L$. inf hamster model at $50 \mathrm{mg} / \mathrm{kg}$ b.i.d. These compounds also showed high potencies against $T$. cruzi, but unexpectedly high levels of hERG inhibition ultimately terminated their development.

Meanwhile, investigation of the C-6 linker group revealed that the parent ether moiety in $\mathrm{R}-\mathbf{1}\left(\mathrm{OCH}_{2}\right)$ was nonoptimal for $\mathrm{VL}$, with shorter and longer chain variants ( $\mathrm{O}$ and $\mathrm{OCH}_{2} \mathrm{C} \equiv \mathrm{C}$, respectively) improving potency against $L$. inf (whereas $\mathrm{O}$-carbamate and $\mathrm{N}$-linked alternatives were poor). Although O-linked phenylpyridine $R-168$ displayed superb activity in the mouse VL model $(99.7 \%$ reduction in parasite burden at $6.25 \mathrm{mg} / \mathrm{kg}$, similar to the case for 4 ), we elected to focus instead on two less lipophilic monoaryl leads (R-6 and pyridine $R-136)$, having superior solubility values $(12-110 \mu \mathrm{g} /$ $\mathrm{mL}$ ), low hERG risk, and excellent PK profiles in three species (mouse, rat, and hamster). Both compounds delivered high efficacies in the chronic infection hamster model $(\geq 97 \%$ inhibition at $25 \mathrm{mg} / \mathrm{kg}$, b.i.d.) and showed weakened binding to plasma proteins, although a positive Ames test for pyridine $R$ 136 dissuaded its further advancement and earmarked R-6 as the favored VL backup candidate to 3 . Finally, like the phenylpyridines, R-6 also demonstrated interesting activity against $T$. cruzi, whereas nitrotriazolooxazine congeners of such leads were less effective. These results provide new insights into the exciting potential of bicyclic nitroimidazoles as novel therapies for the treatment of some challenging neglected diseases.

\section{EXPERIMENTAL SECTION}

Combustion analyses were performed by the Campbell Microanalytical Laboratory, University of Otago, Dunedin, New Zealand. Melting points were determined using an Electrothermal IA9100 melting point apparatus and are as read. NMR spectra were measured on a Bruker Avance 400 spectrometer at $400 \mathrm{MHz}$ for ${ }^{1} \mathrm{H}$ and 100 $\mathrm{MHz}$ for ${ }^{13} \mathrm{C}$ and were referenced to $\mathrm{Me}_{4} \mathrm{Si}$ or solvent resonances. Chemical shifts and coupling constants were recorded in units of parts per million and hertz, respectively. High-resolution electron impact (HREIMS), chemical ionization (HRCIMS), and fast atom bombardment (HRFABMS) mass spectra were recorded on a VG-70SE mass spectrometer at a nominal 5000 resolution. High-resolution electrospray ionization (HRESIMS) mass spectrometry was conducted on a Bruker micrOTOF-Q II mass spectrometer. Low-resolution atmospheric-pressure chemical ionization (APCI) mass spectra were obtained for organic solutions using a ThermoFinnigan Surveyor MSQ mass spectrometer connected to a Gilson autosampler. Optical rotations were measured on a Schmidt + Haensch Polartronic NH8 polarimeter. Column chromatography was performed on silica gel (Merck 230-400 mesh). Chromatographed compounds were typically further purified by crystallization from two solvent combinations, e.g., $\mathrm{CH}_{2} \mathrm{Cl}_{2}$ and $n$-hexane, EtOAc and $n$-hexane, $\mathrm{Et}_{2} \mathrm{O}$ and $n$-pentane, or $\mathrm{CH}_{2} \mathrm{Cl}_{2}$ and $n$-pentane (occasionally, $\mathrm{Et}_{2} \mathrm{O}$ was added to the latter combination to induce solidification, while some compounds required cooling at $\left.-20{ }^{\circ} \mathrm{C}\right)$; more polar compounds were first dissolved in a minimum of $10 \% \mathrm{MeOH} / \mathrm{CH}_{2} \mathrm{Cl}_{2}$ and slowly diluted with $n$-hexane to give the solid product. Thin-layer chromatography was performed on aluminum-backed silica gel plates (Merck $60 \mathrm{~F}_{254}$ ), with visualization of components by UV light $(254 \mathrm{~nm}), \mathrm{I}_{2}$, or $\mathrm{KMnO}_{4}$ staining. Tested compounds (including batches screened in vivo) were all $\geq 95 \%$ pure, as determined by combustion analysis (results within $0.4 \%$ of theoretical values) and/or by HPLC conducted on an Agilent 1100 system with diode array detection, using a $150 \mathrm{~mm} \times 3.2 \mathrm{~mm}$ Altima 5 $\mu \mathrm{m}$ reversed phase $\mathrm{C} 18$ column or a $150 \mathrm{~mm} \times 4.6 \mathrm{~mm}$ Zorbax Eclipse XDB $5 \mu \mathrm{m} \mathrm{C8}$ column and eluting with a gradient (40 to $100 \%$ ) of $80 \% \mathrm{CH}_{3} \mathrm{CN} /$ water in $45 \mathrm{mM}$ ammonium formate buffer ( $\mathrm{pH}$ 3.5). Finally, the chiral purity of lead $\mathrm{R}-6$ was assessed by HPLC performed on a Shimadzu 2010 system with diode array detection, employing a $150 \mathrm{~mm} \times 4.6 \mathrm{~mm}$ CHIRALPAK AY-H $5 \mu \mathrm{m}$ analytical column and isocratic elution with $20 \% \mathrm{EtOH} / n$-heptane.

Compounds of Table 1. The following section details the syntheses of compounds $\mathbf{1 4}, \mathbf{3 9}$, and $\mathbf{4 1}$ of Table 1, via representative procedures and key intermediates, as described in Scheme 1. For the syntheses of the other compounds in Table 1, see the Supporting Information.

Synthesis of 14 (Scheme 1A). Procedure A: 1-[(tertButyldimethylsilyl)oxy]-3-(2,4-dinitro-1 H-imidazol-1-yl)-2-methylpropan-2-ol (10). A mixture of 2,4-dinitro- $1 \mathrm{H}$-imidazole (8) $(1.00 \mathrm{~g}$, $6.33 \mathrm{mmol})$ and tert-butyldimethyl[(2-methyloxiran-2-yl)methoxy]silane ${ }^{33}(9)(1.81 \mathrm{~g}, 8.94 \mathrm{mmol})$ under $\mathrm{N}_{2}$ was stirred at $75^{\circ} \mathrm{C}$ for 18 
h. The resulting cooled mixture was diluted with EtOAc $(250 \mathrm{~mL})$ and washed with aqueous $\mathrm{NaHCO}_{3}(150 \mathrm{~mL})$ and brine $(125 \mathrm{~mL})$, backextracting with EtOAc $(150 \mathrm{~mL})$. The combined extracts were dried $\left(\mathrm{Na}_{2} \mathrm{SO}_{4}\right)$ and then evaporated to dryness under reduced pressure, and the remaining oil was chromatographed on silica gel. Elution with 10$33 \% \mathrm{EtOAc} /$ petroleum ether gave $10(0.92 \mathrm{~g}, 40 \%)$ as a yellow oil: ${ }^{1} \mathrm{H}$ NMR $\left(\mathrm{CDCl}_{3}\right) \delta 7.98(\mathrm{~s}, 1 \mathrm{H}), 4.75(\mathrm{~d}, J=14.0 \mathrm{~Hz}, 1 \mathrm{H}), 4.53(\mathrm{~d}, J=$ $14.0 \mathrm{~Hz}, 1 \mathrm{H}), 3.54(\mathrm{~d}, J=10.2 \mathrm{~Hz}, 1 \mathrm{H}), 3.46(\mathrm{~d}, J=10.1 \mathrm{~Hz}, 1 \mathrm{H})$, $2.52($ br s, $1 \mathrm{H}), 1.17(\mathrm{~s}, 3 \mathrm{H}), 0.91(\mathrm{~s}, 9 \mathrm{H}), 0.09(2 \mathrm{~s}, 2 \times 3 \mathrm{H})$; HRCIMS $\left(\mathrm{NH}_{3}\right)$ calcd for $\mathrm{C}_{13} \mathrm{H}_{25} \mathrm{~N}_{4} \mathrm{O}_{6} \mathrm{Si} m / z[\mathrm{M}+\mathrm{H}]^{+}$361.1543, found 361.1545.

Procedure B: 1-\{3-[(tert-Butyldimethylsilyl)oxy]-2-methyl-2-[(tetrahydro-2H-pyran-2-yl)oxy]propyl\}-2,4-dinitro-1H-imidazole (11). A mixture of alcohol $10(248 \mathrm{mg}, 0.688 \mathrm{mmol}), 3,4$-dihydro- $2 \mathrm{H}$-pyran $(0.32 \mathrm{~mL}, 3.51 \mathrm{mmol})$, and PPTS $(95 \mathrm{mg}, 0.378 \mathrm{mmol})$ in anhydrous $\mathrm{CH}_{2} \mathrm{Cl}_{2}(10 \mathrm{~mL})$ under $\mathrm{N}_{2}$ was stirred at $20^{\circ} \mathrm{C}$ for $5 \mathrm{~h}$. The resulting solution was diluted with $\mathrm{CH}_{2} \mathrm{Cl}_{2}(100 \mathrm{~mL})$ and washed with aqueous $\mathrm{NaHCO}_{3}(2 \times 50 \mathrm{~mL})$, water $(50 \mathrm{~mL})$, and brine $(50 \mathrm{~mL})$, backextracting with $\mathrm{CH}_{2} \mathrm{Cl}_{2}(50 \mathrm{~mL})$. The combined extracts were dried $\left(\mathrm{Na}_{2} \mathrm{SO}_{4}\right)$ and then evaporated to dryness under reduced pressure, and the remaining oil was chromatographed on silica gel. Elution with $10 \%$ EtOAc/petroleum ether gave $\mathbf{1 1}(234 \mathrm{mg}, 76 \%)$ as a yellow oil (a 1:1 mixture of diastereomers): ${ }^{1} \mathrm{H}$ NMR $\left(\mathrm{CDCl}_{3}\right) \delta 8.25,7.99(2 \mathrm{~s}, 1 \mathrm{H})$, $4.88(\mathrm{~d}, J=13.8 \mathrm{~Hz}, 0.5 \mathrm{H}), 4.87(\mathrm{~d}, J=14.0 \mathrm{~Hz}, 0.5 \mathrm{H}), 4.84-4.72$ $(\mathrm{m}, 1 \mathrm{H}), 4.62(\mathrm{~d}, J=14.0 \mathrm{~Hz}, 0.5 \mathrm{H}), 4.54(\mathrm{~d}, J=13.8 \mathrm{~Hz}, 0.5 \mathrm{H})$, $3.73-3.65(\mathrm{~m}, 1 \mathrm{H}), 3.60-3.36(\mathrm{~m}, 2 \mathrm{H}), 3.29(\mathrm{~d}, J=10.6 \mathrm{~Hz}, 0.5 \mathrm{H})$, $3.26(\mathrm{~d}, J=10.7 \mathrm{~Hz}, 0.5 \mathrm{H}), 1.95-1.35(\mathrm{~m}, 6 \mathrm{H}), 1.33,1.25(2 \mathrm{~s}, 3 \mathrm{H})$, 0.94, $0.87(2 \mathrm{~s}, 9 \mathrm{H}), 0.10(2 \mathrm{~s}, 3 \mathrm{H}), 0.04,0.02(2 \mathrm{~s}, 3 \mathrm{H})$; HRCIMS $\left(\mathrm{NH}_{3}\right)$ calcd for $\mathrm{C}_{18} \mathrm{H}_{33} \mathrm{~N}_{4} \mathrm{O}_{7} \mathrm{Si} m / z[\mathrm{M}+\mathrm{H}]^{+}$445.2119, found 445.2125.

Procedure C: 6-Methyl-2-nitro-6-[(tetrahydro-2H-pyran-2-yl)oxy]6,7-dihydro-5H-imidazo[2,1-b][1,3]oxazine (12). A solution of silyl ether 11 (230 mg, $0.517 \mathrm{mmol})$ in anhydrous THF $(6 \mathrm{~mL})$ under $\mathrm{N}_{2}$ was treated with TBAF $(1.5 \mathrm{~mL}$ of a $1 \mathrm{M}$ solution in THF, 1.50 $\mathrm{mmol}$ ), and the mixture was stirred at $20^{\circ} \mathrm{C}$ for $1 \mathrm{~h}$. The resulting solution was concentrated under reduced pressure, then diluted with EtOAc $(50 \mathrm{~mL})$, and washed with aqueous $\mathrm{NaHCO}_{3}(50 \mathrm{~mL})$ and brine $(50 \mathrm{~mL})$, back-extracting with EtOAc $(2 \times 50 \mathrm{~mL})$. The combined extracts were dried $\left(\mathrm{Na}_{2} \mathrm{SO}_{4}\right)$ and then evaporated to dryness under reduced pressure, and the residue was chromatographed on silica gel. Elution with $5 \% \mathrm{MeOH} / \mathrm{CH}_{2} \mathrm{Cl}_{2}$ gave 12 (130 mg, 89\%) as a white solid (a $1: 1$ mixture of diastereomers): $\mathrm{mp}\left(\mathrm{CH}_{2} \mathrm{Cl}_{2}\right.$ / hexane) $153-156{ }^{\circ} \mathrm{C} ;{ }^{1} \mathrm{H}$ NMR $\left(\mathrm{CDCl}_{3}\right) \delta 7.40,7.37(2 \mathrm{~s}, 1 \mathrm{H})$, $5.03-4.95(\mathrm{~m}, 1 \mathrm{H}), 4.54(\mathrm{dd}, J=11.6,2.3 \mathrm{~Hz}, 0.5 \mathrm{H}), 4.44(\mathrm{dd}, J=$ $11.8,2.7 \mathrm{~Hz}, 0.5 \mathrm{H}), 4.34(\mathrm{dd}, J=12.7,2.7 \mathrm{~Hz}, 0.5 \mathrm{H}), 4.18(\mathrm{~d}, J=$ $11.6 \mathrm{~Hz}, 0.5 \mathrm{H}), 4.11(\mathrm{~d}, J=11.7 \mathrm{~Hz}, 0.5 \mathrm{H}), 4.09(\mathrm{dd}, J=12.5,2.3$ $\mathrm{Hz}, 0.5 \mathrm{H}), 3.89$ (d, $J=12.6 \mathrm{~Hz}, 0.5 \mathrm{H}), 3.89-3.81(\mathrm{~m}, 1 \mathrm{H}), 3.57-$ $3.44(\mathrm{~m}, 1 \mathrm{H}), 3.42-3.33(\mathrm{~m}, 0.5 \mathrm{H}), 1.92-1.38(\mathrm{~m}, 9 \mathrm{H})$; APCI MS $\mathrm{m} / z 284[\mathrm{M}+\mathrm{H}]^{+}$.

Procedure D: 6-Methyl-2-nitro-6,7-dihydro-5H-imidazo[2,1-b][1,3]oxazin-6-ol (13). A solution of THP ether $12(120 \mathrm{mg}, 0.424$ $\mathrm{mmol})$ in $\mathrm{MeOH}(10 \mathrm{~mL})$ was treated with methanesulfonic acid $(52.6 \mathrm{mg}, 0.547 \mathrm{mmol})$, and the mixture was stirred at $20^{\circ} \mathrm{C}$ for $1 \mathrm{~h}$. The resulting solution was neutralized with aqueous $\mathrm{NaHCO}_{3}$; then the solvents were removed under reduced pressure, and the residue was chromatographed on silica gel. Elution with $5 \% \mathrm{MeOH} / \mathrm{CH}_{2} \mathrm{Cl}_{2}$ gave $13(80 \mathrm{mg}, 95 \%)$ as a white solid: $\mathrm{mp}\left(\mathrm{MeOH} / \mathrm{CH}_{2} \mathrm{Cl}_{2} /\right.$ hexane $)$ $185-188{ }^{\circ} \mathrm{C} ;{ }^{1} \mathrm{H}$ NMR $\left[\left(\mathrm{CD}_{3}\right)_{2} \mathrm{SO}\right] \delta 8.05(\mathrm{~s}, 1 \mathrm{H}), 5.50($ br s, $1 \mathrm{H})$, $4.26(\mathrm{~d}, J=11.1 \mathrm{~Hz}, 1 \mathrm{H}), 4.12(\mathrm{dd}, J=11.1,2.6 \mathrm{~Hz}, 1 \mathrm{H}), 4.00(\mathrm{~d}, J=$ $12.6 \mathrm{~Hz}, 1 \mathrm{H}), 3.89$ (dd, $J=12.6,2.4 \mathrm{~Hz}, 1 \mathrm{H}), 1.24(\mathrm{~s}, 3 \mathrm{H})$; HREIMS calcd for $\mathrm{C}_{7} \mathrm{H}_{9} \mathrm{~N}_{3} \mathrm{O}_{4} \mathrm{~m} / z\left(\mathrm{M}^{+}\right)$199.0593, found 199.0591 .

Procedure E: 6-Methyl-2-nitro-6-\{[4-(trifluoromethoxy)benzyl]oxy\}-6,7-dihydro-5H-imidazo[2,1-b][1,3]oxazine (14). A solution of alcohol 13 (50.7 mg, $0.255 \mathrm{mmol}$ ) and 4-(trifluoromethoxy)benzyl bromide $(65 \mu \mathrm{L}, 0.406 \mathrm{mmol})$ in anhydrous DMF $(3 \mathrm{~mL})$ under $\mathrm{N}_{2}$ was treated with $60 \% \mathrm{NaH}(20 \mathrm{mg}, 0.50 \mathrm{mmol})$, and the mixture was stirred at $20^{\circ} \mathrm{C}$ for $3 \mathrm{~h}$. The resulting mixture was diluted with EtOAc $(150 \mathrm{~mL})$ and washed with aqueous $\mathrm{NaHCO}_{3}(50 \mathrm{~mL})$, water $(2 \times 50$ $\mathrm{mL})$, and brine $(50 \mathrm{~mL})$, back-extracting with EtOAc $(100 \mathrm{~mL})$. The combined extracts were dried $\left(\mathrm{Na}_{2} \mathrm{SO}_{4}\right)$ and then evaporated to dryness under reduced pressure, and the residue was chromatographed on silica gel. Elution with $1-2 \% \mathrm{MeOH} / \mathrm{CH}_{2} \mathrm{Cl}_{2}$ gave 14 (45 mg, $47 \%$ ) as a white solid: $\mathrm{mp}$ (EtOAc/hexane) $139-141{ }^{\circ} \mathrm{C}$; ${ }^{1} \mathrm{H}$ NMR $\left[\left(\mathrm{CD}_{3}\right)_{2} \mathrm{SO}\right] \delta 7.99(\mathrm{~s}, 1 \mathrm{H}), 7.31(\mathrm{br} \mathrm{d}, J=9.0 \mathrm{~Hz}, 2 \mathrm{H}), 7.28(\mathrm{br} \mathrm{d}, J$ $=9.0 \mathrm{~Hz}, 2 \mathrm{H}), 4.60(\mathrm{dd}, J=11.9,2.8 \mathrm{~Hz}, 1 \mathrm{H}), 4.56(\mathrm{br} \mathrm{s}, 2 \mathrm{H}), 4.37$ $(\mathrm{d}, J=11.8 \mathrm{~Hz}, 1 \mathrm{H}), 4.24(\mathrm{dd}, J=13.2,2.6 \mathrm{~Hz}, 1 \mathrm{H}), 4.07(\mathrm{~d}, J=13.1$ $\mathrm{Hz}, 1 \mathrm{H}), 1.36(\mathrm{~s}, 3 \mathrm{H}) ;{ }^{13} \mathrm{C} \mathrm{NMR}\left[\left(\mathrm{CD}_{3}\right)_{2} \mathrm{SO}\right] \delta 147.5,147.0,142.2$, $137.8,129.0(2 \mathrm{C}), 120.9(2 \mathrm{C}), 120.0\left(\mathrm{q}, J_{\mathrm{C}-\mathrm{F}}=255.9 \mathrm{~Hz}\right), 117.8$, 70.6, 69.2, 62.9, 50.7, 17.0. Anal. Calcd for $\mathrm{C}_{15} \mathrm{H}_{14} \mathrm{~F}_{3} \mathrm{~N}_{3} \mathrm{O}_{5}$ : C, 48.26; $\mathrm{H}, 3.78$; N, 11.26. Found: C, 48.23; H, 3.98; N, 11.31 .

Synthesis of 39 and 41 (Scheme 1D). 2-Bromo-4-nitro-1-\{3[(tetrahydro-2H-pyran-2-yl)oxy]-4-[(triisopropylsilyl)oxy]butyl\}- $1 \mathrm{H}$ imidazole (35). Reaction of 4-(2-bromo-4-nitro-1H-imidazol-1-yl)-1[(triisopropylsilyl)oxy]butan-2-ol ${ }^{24}$ (34) with 3,4-dihydro-2H-pyran (4.0 equiv) and PPTS, using procedure $B$ for 1 day (but washing with aqueous $\mathrm{NaHCO}_{3}$ only and extracting the product four times with $\mathrm{CH}_{2} \mathrm{Cl}_{2}$ ), followed by chromatography of the product on silica gel, eluting with $25-50 \% \mathrm{CH}_{2} \mathrm{Cl}_{2}$ /petroleum ether (forerunners) and then with $\mathrm{CH}_{2} \mathrm{Cl}_{2}$, gave $35(100 \%)$ as a colorless oil (a $1: 1$ mixture of diastereomers): ${ }^{1} \mathrm{H}$ NMR $\left(\mathrm{CDCl}_{3}\right) \delta 8.20,7.82(2 \mathrm{~s}, 1 \mathrm{H}), 4.69-4.58$ (m, $1 \mathrm{H}), 4.31-4.10(\mathrm{~m}, 2 \mathrm{H}), 4.08-3.99(\mathrm{~m}, 1 \mathrm{H}), 3.92-3.43(\mathrm{~m}, 4$ $\mathrm{H}), 2.34-2.23(\mathrm{~m}, 0.5 \mathrm{H}), 2.12-2.01(\mathrm{~m}, 1 \mathrm{H}), 1.94-1.41(\mathrm{~m}, 6.5 \mathrm{H})$, 1.18-0.96 (m, $21 \mathrm{H})$; HRFABMS calcd for $\mathrm{C}_{21} \mathrm{H}_{39} \mathrm{BrN}_{3} \mathrm{O}_{5} \mathrm{Si} m / z[\mathrm{M}$ $+\mathrm{H}]^{+}$522.1822, 520.1842, found 522.1838, 520.1826.

4-(2-Bromo-4-nitro-1H-imidazol-1-yl)-2-[(tetrahydro-2H-pyran2-yl)oxy]butan-1-ol (36). Reaction of silyl ether 35 with TBAF (1.1 equiv), using procedure $\mathrm{C}$ for $3 \mathrm{~h}$ (extracting the product five times with EtOAc), followed by chromatography of the product on silica gel, eluting with 33\% EtOAc/petroleum ether (forerunners) and then with $33-50 \% \mathrm{EtOAc} /$ petroleum ether, gave $36(91 \%)$ as a white solid (a 3:1 mixture of diastereomers): $\mathrm{mp}\left(\mathrm{Et}_{2} \mathrm{O} / \mathrm{CH}_{2} \mathrm{Cl}_{2} /\right.$ pentane $)$ 89-91 ${ }^{\circ} \mathrm{C}$; ${ }^{1} \mathrm{H}$ NMR $\left(\mathrm{CDCl}_{3}\right) \delta 8.16,7.79(2 \mathrm{~s}, 1 \mathrm{H}), 4.65-4.50(\mathrm{~m}, 1 \mathrm{H})$, 4.29-4.12 (m, $2 \mathrm{H}), 4.08-3.97(\mathrm{~m}, 1 \mathrm{H}), 3.85-3.46(\mathrm{~m}, 4 \mathrm{H}), 2.12-$ $1.73(\mathrm{~m}, 5 \mathrm{H}), 1.65-1.45(\mathrm{~m}, 4 \mathrm{H})$. Anal. Calcd for $\mathrm{C}_{12} \mathrm{H}_{18} \mathrm{BrN}_{3} \mathrm{O}_{5}: \mathrm{C}$, 39.58; H, 4.98; N, 11.54. Found: C, 39.85; H, 5.12; N, 11.57.

Procedure F: 2-Nitro-7-[(tetrahydro-2H-pyran-2-yl)oxy]-5,6,7,8tetrahydroimidazo[2,1-b][1,3]oxazepine (37). A solution of alcohol $36(730 \mathrm{mg}, 2.00 \mathrm{mmol})$ in anhydrous DMF $(8 \mathrm{~mL})$ under $\mathrm{N}_{2}$ at $0{ }^{\circ} \mathrm{C}$ was treated with $60 \% \mathrm{NaH}(148 \mathrm{mg}, 3.70 \mathrm{mmol})$ and then quickly degassed and resealed under $\mathrm{N}_{2}$. The mixture was stirred at $20{ }^{\circ} \mathrm{C}$ for $3.5 \mathrm{~h}$ and then cooled to $-78{ }^{\circ} \mathrm{C}\left(\mathrm{CO}_{2} /\right.$ acetone $)$, the reaction quenched with ice/aqueous $\mathrm{NaHCO}_{3}(50 \mathrm{~mL})$, and the mixture extracted with EtOAc $(7 \times 50 \mathrm{~mL})$. The extracts were washed with brine $(50 \mathrm{~mL})$ and then evaporated to dryness under reduced pressure (at $30^{\circ} \mathrm{C}$ ), and the residue was chromatographed on silica gel. Elution with $0-33 \% \mathrm{EtOAc/petroleum} \mathrm{ether} \mathrm{first} \mathrm{gave} \mathrm{forerunners,} \mathrm{and} \mathrm{then}$ further elution with 50\% EtOAc/petroleum ether gave the crude product, which was chromatographed again on silica gel. Elution with $0-2 \% \mathrm{EtOAc} / \mathrm{CH}_{2} \mathrm{Cl}_{2}$ first gave forerunners, and then further elution with $3-4 \% \mathrm{EtOAc} / \mathrm{CH}_{2} \mathrm{Cl}_{2}$ gave $37(144 \mathrm{mg}, 25 \%)$ as a white solid: $\mathrm{mp}\left(\mathrm{CH}_{2} \mathrm{Cl}_{2} /\right.$ hexane $) 158-160{ }^{\circ} \mathrm{C}$; ${ }^{1} \mathrm{H}$ NMR $\left(\mathrm{CDCl}_{3}\right) \delta 7.48(\mathrm{~s}, 1$ $\mathrm{H})$, 4.83-4.75 (m, $1 \mathrm{H}), 4.39-4.04(\mathrm{~m}, 4 \mathrm{H}), 3.96-3.82(\mathrm{~m}, 2 \mathrm{H})$ $3.60-3.50(\mathrm{~m}, 1 \mathrm{H}), 2.25-2.02(\mathrm{~m}, 2 \mathrm{H}), 1.92-1.71(\mathrm{~m}, 2 \mathrm{H}), 1.69-$ $1.50(\mathrm{~m}, 4 \mathrm{H})$. Anal. Calcd for $\mathrm{C}_{12} \mathrm{H}_{17} \mathrm{~N}_{3} \mathrm{O}_{5}: \mathrm{C}, 50.88 ; \mathrm{H}, 6.05 ; \mathrm{N}$, 14.83. Found: C, 51.08; H, 6.08; N, 14.89 .

2-Nitro-5,6,7,8-tetrahydroimidazo[2,1-b][1,3]oxazepin-7-ol (38). A solution of THP ether $37(137 \mathrm{mg}, 0.484 \mathrm{mmol})$ in $\mathrm{MeOH}(14$ $\mathrm{mL})$ was treated with $3.3 \mathrm{M} \mathrm{HCl}(0.47 \mathrm{~mL}, 1.55 \mathrm{mmol})$. The mixture was stirred at $20{ }^{\circ} \mathrm{C}$ for $5 \mathrm{~h}$, cooled to $-20{ }^{\circ} \mathrm{C}$, and neutralized with a solution of $\mathrm{NH}_{3}$ in $\mathrm{MeOH}(0.8 \mathrm{~mL}$ of a $7 \mathrm{M}$ solution). The resulting mixture was evaporated to dryness under reduced pressure (at $30^{\circ} \mathrm{C}$ ), and the residue was chromatographed on silica gel. Elution with $0-$ $25 \% \mathrm{EtOAc} / \mathrm{CH}_{2} \mathrm{Cl}_{2}$ first gave forerunners, and then further elution with $25 \% \mathrm{EtOAc} / \mathrm{CH}_{2} \mathrm{Cl}_{2}$ gave 38 (91 mg, $94 \%$ ) as a white solid: $\mathrm{mp}$ $\left(\mathrm{MeOH} / \mathrm{CH}_{2} \mathrm{Cl}_{2} /\right.$ hexane $) 185-187^{\circ} \mathrm{C}$; ${ }^{1} \mathrm{H}$ NMR $\left[\left(\mathrm{CD}_{3}\right)_{2} \mathrm{SO}\right] \delta 8.15$ (s, $1 \mathrm{H}), 5.28$ (br s, $1 \mathrm{H}), 4.22$ (ddd, $J=14.1,9.1,2.0 \mathrm{~Hz}, 1 \mathrm{H}), 4.19-$ $4.09(\mathrm{~m}, 1 \mathrm{H}), 4.02-3.91(\mathrm{~m}, 3 \mathrm{H}), 2.09-1.98(\mathrm{~m}, 1 \mathrm{H}), 1.88-1.76$ $(\mathrm{m}, 1 \mathrm{H})$. Anal. Calcd for $\mathrm{C}_{7} \mathrm{H}_{9} \mathrm{~N}_{3} \mathrm{O}_{4}: \mathrm{C}, 42.21 ; \mathrm{H}, 4.55 ; \mathrm{N}, 21.10$. Found: C, 42.41; H, 4.72; N, 21.28 . 
Procedure G: 2-Nitro-7-\{[4-(trifluoromethoxy)benzyl]oxy\}-5,6,7,8tetrahydroimidazo[2,1-b][1,3]oxazepine (39) and 2-Nitro-7-(\{[4(trifluoromethoxy)benzyl]oxy\}methyl)-6,7-dihydro-5H-imidazo[2, 1 b][1,3]oxazine (41). A mixture of alcohol $38(51.0 \mathrm{mg}, 0.256 \mathrm{mmol})$ and 4-(trifluoromethoxy)benzyl bromide $(0.205 \mathrm{~mL}, 1.28 \mathrm{mmol})$ in anhydrous DMF $(1.5 \mathrm{~mL})$ under $\mathrm{N}_{2}$ at $0{ }^{\circ} \mathrm{C}$ was treated with $60 \%$ $\mathrm{NaH}(17.5 \mathrm{mg}, 0.438 \mathrm{mmol})$ and then quickly degassed and resealed under $\mathrm{N}_{2}$. The mixture was stirred at $20{ }^{\circ} \mathrm{C}$ for $130 \mathrm{~min}$ and then cooled to $-78{ }^{\circ} \mathrm{C}\left(\mathrm{CO}_{2}\right.$ /acetone), the reaction quenched with ice/ aqueous $\mathrm{NaHCO}_{3}(10 \mathrm{~mL})$, and the mixture added to brine $(40 \mathrm{~mL})$ and extracted with $\mathrm{CH}_{2} \mathrm{Cl}_{2}(5 \times 50 \mathrm{~mL})$. The combined extracts were evaporated to dryness under reduced pressure (at $30{ }^{\circ} \mathrm{C}$ ), and the residue was chromatographed on silica gel. Elution with $0-50 \%$ EtOAc/petroleum ether first gave forerunners, and then further elution with $50 \% \mathrm{EtOAc} /$ petroleum ether gave $39(37 \mathrm{mg}, 39 \%)$ as a white solid: $\mathrm{mp}\left(\mathrm{CH}_{2} \mathrm{Cl}_{2} /\right.$ pentane $)$ 119-121 ${ }^{\circ} \mathrm{C} ;{ }^{1} \mathrm{H}$ NMR $\left[\left(\mathrm{CD}_{3}\right)_{2} \mathrm{SO}\right] \delta 8.18(\mathrm{~s}, 1 \mathrm{H}), 7.51(\mathrm{br} \mathrm{d}, J=8.8 \mathrm{~Hz}, 2 \mathrm{H}), 7.36(\mathrm{br}$ d, $J=7.9 \mathrm{~Hz}, 2 \mathrm{H}), 4.66(\mathrm{~s}, 2 \mathrm{H}), 4.36(\mathrm{dd}, J=12.6,4.6 \mathrm{~Hz}, 1 \mathrm{H})$, $4.26-4.13(\mathrm{~m}, 2 \mathrm{H}), 4.04$ (ddd, $J=14.2,6.4,3.0 \mathrm{~Hz}, 1 \mathrm{H}), 3.95-3.86$ $(\mathrm{m}, 1 \mathrm{H}), 2.19-2.02(\mathrm{~m}, 2 \mathrm{H}) ;{ }^{13} \mathrm{C}$ NMR $\left[\left(\mathrm{CD}_{3}\right)_{2} \mathrm{SO}\right] \delta$ 151.1, 147.6 $\left(\mathrm{q}, J_{\mathrm{C}-\mathrm{F}}=1.5 \mathrm{~Hz}\right), 140.6,137.9,129.3(2 \mathrm{C}), 120.9$ (2 C), 120.4, 120.1 $\left(\mathrm{q}, J_{\mathrm{C}-\mathrm{F}}=256.1 \mathrm{~Hz}\right), 74.5,73.3,68.7,42.0,30.1$. Anal. Calcd for $\mathrm{C}_{15} \mathrm{H}_{14} \mathrm{~F}_{3} \mathrm{~N}_{3} \mathrm{O}_{5} \cdot 0.5 \mathrm{H}_{2} \mathrm{O}: \mathrm{C}, 47.13 ; \mathrm{H}, 3.96 ; \mathrm{N}, 10.99$. Found: $\mathrm{C}, 46.86$; $\mathrm{H}, 3.63$; N, 10.82. HPLC purity of $100 \%$.

Further elution of the column described above with $50-75 \%$ $\mathrm{EtOAc/petroleum} \mathrm{ether} \mathrm{gave} \mathrm{crude} \mathrm{41,} \mathrm{which} \mathrm{was} \mathrm{chromatographed}$ again on silica gel (as before) to give $\mathbf{4 1}^{24}(34 \mathrm{mg}, 36 \%$ ) as a cream solid: $\mathrm{mp}$ (EtOAc/hexane) $163-164{ }^{\circ} \mathrm{C}$ (lit. $\left.{ }^{24} \mathrm{mp} 158-160{ }^{\circ} \mathrm{C}\right) ;{ }^{1} \mathrm{H}$ $\operatorname{NMR}\left(\mathrm{CDCl}_{3}\right) \delta 7.42(\mathrm{~s}, 1 \mathrm{H}), 7.34(\mathrm{br} \mathrm{d}, J=8.7 \mathrm{~Hz}, 2 \mathrm{H}), 7.20$ (br d, $J=8.6 \mathrm{~Hz}, 2 \mathrm{H}), 4.61(\mathrm{~s}, 2 \mathrm{H}), 4.61-4.54(\mathrm{~m}, 1 \mathrm{H}), 4.15$ (ddd, $J=$ 12.4, 5.7, $3.7 \mathrm{~Hz}, 1 \mathrm{H}$ ), 4.06 (ddd, $J=12.4,10.0,5.8 \mathrm{~Hz}, 1 \mathrm{H}$ ), 3.83 (dd, $J=10.6,4.4 \mathrm{~Hz}, 1 \mathrm{H}), 3.78$ (dd, $J=10.7,4.9 \mathrm{~Hz}, 1 \mathrm{H}), 2.39-2.21$ $(\mathrm{m}, 2 \mathrm{H})$; APCI MS $m / z 374[\mathrm{M}+\mathrm{H}]^{+}$; HPLC purity of $100 \%$.

Compounds of Table 2. The following section details the syntheses of compounds $R-58$ and $R-69$ of Table 2, via representative procedures and key intermediates, as described in Scheme 2. For the syntheses of the other compounds in Table 2, see the Supporting Information.

Synthesis of R-58 (Scheme 2A). (6R)-6-[(2-lodobenzyl)oxy]-2nitro-6,7-dihydro-5H-imidazo[2,1-b][1,3]oxazine (57). Reaction of (6R)-2-nitro-6,7-dihydro-5H-imidazo[2,1-b][1,3] oxazin-6-ol $^{32}$ (56) with 2-iodobenzyl chloride (3.0 equiv) and $\mathrm{NaH}$ (1.6 equiv), using procedure $\mathrm{G}$ for $3.5 \mathrm{~h}$, followed by chromatography of the product on silica gel, eluting with $0-0.25 \% \mathrm{MeOH} / \mathrm{CH}_{2} \mathrm{Cl}_{2}$ (forerunners) and then with $0.25-0.3 \% \mathrm{MeOH} / \mathrm{CH}_{2} \mathrm{Cl}_{2}$, gave $\mathbf{5 7}(91 \%)$ as a light yellow solid: $\mathrm{mp}\left(\mathrm{Et}_{2} \mathrm{O}\right) 118-119{ }^{\circ} \mathrm{C} ;{ }^{1} \mathrm{H}$ NMR $\left(\mathrm{CDCl}_{3}\right) \delta 7.85$ (br d, $J=7.8$ $\mathrm{Hz}, 1 \mathrm{H}), 7.40$ (s, $1 \mathrm{H}), 7.37-7.33(\mathrm{~m}, 2 \mathrm{H}), 7.08-7.00(\mathrm{~m}, 1 \mathrm{H}), 4.72$ $(\mathrm{d}, J=12.0 \mathrm{~Hz}, 1 \mathrm{H}), 4.72-4.65(\mathrm{~m}, 1 \mathrm{H}), 4.64(\mathrm{~d}, J=12.0 \mathrm{~Hz}, 1 \mathrm{H})$, 4.40 (br dd, $J=12.1,0.9 \mathrm{~Hz}, 1 \mathrm{H}), 4.25-4.19(\mathrm{~m}, 3 \mathrm{H})$. Anal. Calcd for $\mathrm{C}_{13} \mathrm{H}_{12} \mathrm{IN}_{3} \mathrm{O}_{4}$ : C, 38.92; H, 3.02; N, 10.47. Found: C, 39.08; H, 2.98; N, 10.44 .

Procedure H: (6R)-2-Nitro-6-\{[4'-(trifluoromethoxy)(1,1'-biphenyl)-2-yl]methoxy\}-6,7-dihydro-5H-imidazo[2,1-b][1,3]oxazine (R58). A mixture of iodide $57(435 \mathrm{mg}, 1.08 \mathrm{mmol})$, [4(trifluoromethoxy)phenyl]boronic acid (401 $\mathrm{mg}, 1.95 \mathrm{mmol}$ ), and $\mathrm{Pd}(\mathrm{dppf}) \mathrm{Cl}_{2}(56 \mathrm{mg}, 0.077 \mathrm{mmol})$ in $\mathrm{DMF}(6 \mathrm{~mL})$ and aqueous $\mathrm{KHCO}_{3}(2 \mathrm{~mL}$ of a $2 \mathrm{M}$ solution, $4.0 \mathrm{mmol})$ was degassed, and then $\mathrm{N}_{2}$ was added. The resulting mixture was stirred at $75^{\circ} \mathrm{C}$ for $4 \mathrm{~h}$, and then cooled, diluted with brine $(100 \mathrm{~mL})$, and extracted with $\mathrm{CH}_{2} \mathrm{Cl}_{2}$ $(3 \times 100 \mathrm{~mL})$. The extracts were evaporated to dryness under reduced pressure, and the residue was chromatographed on silica gel. Elution with $\mathrm{CH}_{2} \mathrm{Cl}_{2}$ first gave forerunners, and then further elution with $2 \%$ $\mathrm{MeOH} / \mathrm{CH}_{2} \mathrm{Cl}_{2}$ gave $\mathrm{R}-\mathbf{5 8}(218 \mathrm{mg}, 46 \%)$ as a cream solid: $\mathrm{mp}$ $\left(\mathrm{CH}_{2} \mathrm{Cl}_{2} /\right.$ hexane $) 133-135{ }^{\circ} \mathrm{C} ;{ }^{1} \mathrm{H}$ NMR $\left[\left(\mathrm{CD}_{3}\right)_{2} \mathrm{SO}\right] \delta 8.00$ (s, 1 $\mathrm{H}), 7.50-7.44(\mathrm{~m}, 3 \mathrm{H}), 7.44-7.35(\mathrm{~m}, 4 \mathrm{H}), 7.33-7.26(\mathrm{~m}, 1 \mathrm{H})$, 4.57-4.49 (m, $2 \mathrm{H}), 4.49(\mathrm{~d}, J=10.9 \mathrm{~Hz}, 1 \mathrm{H}), 4.42(\mathrm{br} \mathrm{d}, J=11.8$ $\mathrm{Hz}, 1 \mathrm{H}), 4.22-4.12(\mathrm{~m}, 3 \mathrm{H}) ;{ }^{13} \mathrm{C} \mathrm{NMR}\left[\left(\mathrm{CD}_{3}\right)_{2} \mathrm{SO}\right] \delta 147.6(\mathrm{q}$, $\left.J_{\mathrm{C}-\mathrm{F}}=1.5 \mathrm{~Hz}\right), 147.1,142.1,140.0,139.3,134.5,130.7$ (2 C), 130.0, $129.9,128.3,127.9,120.6(2 \mathrm{C}), 120.1\left(\mathrm{q}, J_{\mathrm{C}-\mathrm{F}}=256.4 \mathrm{~Hz}\right), 117.9$,
68.1, 67.8, 66.8, 46.5. Anal. Calcd for $\mathrm{C}_{20} \mathrm{H}_{16} \mathrm{~F}_{3} \mathrm{~N}_{3} \mathrm{O}_{5}$ : C, 55.18; $\mathrm{H}$, 3.70; N, 9.65. Found: C, 55.08; H, 3.66; N, 9.68.

Synthesis of R-69 (Scheme 2B). (6R)-6-[(2-Bromopyridin-3yl)methoxy]-2-nitro-6,7-dihydro-5H-imidazo[2,1-b][1,3]oxazine (68). Reaction of alcohol $56^{32}$ with 2-bromo-3-(bromomethyl)pyridine (66) (1.6 equiv) and $\mathrm{NaH}$ (1.6 equiv), using procedure $\mathrm{G}$ at $0{ }^{\circ} \mathrm{C}$ for $1 \mathrm{~h}$ and then at $20^{\circ} \mathrm{C}$ for $80 \mathrm{~min}$, followed by chromatography of the product on silica gel, eluting with $0-0.5 \% \mathrm{MeOH} / \mathrm{CH}_{2} \mathrm{Cl}_{2}$ (forerunners) and then with $0.5-0.67 \% \mathrm{MeOH} / \mathrm{CH}_{2} \mathrm{Cl}_{2}$, gave 68 (83\%) as a cream solid: $\mathrm{mp}\left(\mathrm{MeOH} / \mathrm{CH}_{2} \mathrm{Cl}_{2} /\right.$ hexane $) 189-190{ }^{\circ} \mathrm{C}$; ${ }^{1} \mathrm{H} \mathrm{NMR}\left[\left(\mathrm{CD}_{3}\right)_{2} \mathrm{SO}\right] \delta 8.32(\mathrm{dd}, J=4.7,2.0 \mathrm{~Hz}, 1 \mathrm{H}), 8.05(\mathrm{~s}, 1 \mathrm{H})$, $7.80(\mathrm{dd}, J=7.5,2.0 \mathrm{~Hz}, 1 \mathrm{H}), 7.46(\mathrm{dd}, J=7.5,4.7 \mathrm{~Hz}, 1 \mathrm{H}), 4.73-$ $4.66(\mathrm{~m}, 3 \mathrm{H}), 4.51$ (br d, $J=11.7 \mathrm{~Hz}, 1 \mathrm{H}), 4.39-4.32(\mathrm{~m}, 2 \mathrm{H}), 4.25$ (dd, $J=13.7,3.4 \mathrm{~Hz}, 1 \mathrm{H})$. Anal. Calcd for $\mathrm{C}_{12} \mathrm{H}_{11} \mathrm{BrN}_{4} \mathrm{O}_{4}$ : C, 40.58; $\mathrm{H}, 3.12$; N, 15.78. Found: C, 40.81; H, 3.20; N, 15.72 .

Procedure I: (6R)-2-Nitro-6-(\{2-[4-(trifluoromethoxy)phenyl]pyridin-3-yl\}methoxy)-6,7-dihydro-5H-imidazo[2,1-b][1,3]oxazine (R-69). A stirred mixture of bromide $68(60.3 \mathrm{mg}, 0.170 \mathrm{mmol})$, [4(trifluoromethoxy)phenyl]boronic acid $(60.5 \mathrm{mg}, 0.294 \mathrm{mmol})$, and $\mathrm{Pd}(\mathrm{dppf}) \mathrm{Cl}_{2}(29.7 \mathrm{mg}, 0.041 \mathrm{mmol})$ in DMF $(1.5 \mathrm{~mL})$, toluene $(1.0$ $\mathrm{mL}$ ), and EtOH (0.8 mL) was degassed for $7 \mathrm{~min}$ (vacuum pump), and then $\mathrm{N}_{2}$ was added. An aqueous solution of $\mathrm{Na}_{2} \mathrm{CO}_{3}(0.45 \mathrm{~mL}$ of a $2 \mathrm{M}$ solution, $0.90 \mathrm{mmol}$ ) was added by syringe; the stirred mixture was again degassed for $8 \mathrm{~min}$, and then $\mathrm{N}_{2}$ was added. The resulting mixture was stirred at $87^{\circ} \mathrm{C}$ for $190 \mathrm{~min}$ and then cooled, diluted with aqueous $\mathrm{NaHCO}_{3}(50 \mathrm{~mL})$, and extracted with $\mathrm{CH}_{2} \mathrm{Cl}_{2}(5 \times 50 \mathrm{~mL})$. The combined extracts were evaporated to dryness under reduced pressure $\left(\right.$ at $30{ }^{\circ} \mathrm{C}$ ), and the residue was chromatographed on silica gel. Elution with $0-0.5 \% \mathrm{MeOH} / \mathrm{CH}_{2} \mathrm{Cl}_{2}$ first gave forerunners, and then further elution with $0.5-0.75 \% \mathrm{MeOH} / \mathrm{CH}_{2} \mathrm{Cl}_{2}$ gave $R-69$ (62 $\mathrm{mg}, 84 \%)$ as a pale yellow solid: $\mathrm{mp}\left(\mathrm{MeOH} / \mathrm{CH}_{2} \mathrm{Cl}_{2} /\right.$ hexane $) 246-$ $248{ }^{\circ} \mathrm{C}$; ${ }^{1} \mathrm{H}$ NMR $\left[\left(\mathrm{CD}_{3}\right)_{2} \mathrm{SO}\right] \delta 8.62(\mathrm{dd}, J=4.7,1.7 \mathrm{~Hz}, 1 \mathrm{H}), 8.02$ $(\mathrm{s}, 1 \mathrm{H}), 7.89$ (dd, $J=7.8,1.6 \mathrm{~Hz}, 1 \mathrm{H}), 7.67(\mathrm{br} \mathrm{d}, J=8.8 \mathrm{~Hz}, 2 \mathrm{H})$, 7.43 (dd, $J=7.7,4.8 \mathrm{~Hz}, 1 \mathrm{H}), 7.41($ br d, $J=9.0 \mathrm{~Hz}, 2 \mathrm{H}), 4.68-4.57$ $(\mathrm{m}, 3 \mathrm{H}), 4.46(\mathrm{br} \mathrm{d}, J=11.9 \mathrm{~Hz}, 1 \mathrm{H}), 4.31-4.17(\mathrm{~m}, 3 \mathrm{H}) ;{ }^{13} \mathrm{C}$ NMR $\left[\left(\mathrm{CD}_{3}\right)_{2} \mathrm{SO}\right] \delta 156.2,148.9,148.4,147.1,142.1,138.5,138.2$, $130.8(2 \mathrm{C}), 130.5,122.8,120.4(2 \mathrm{C}), 120.1\left(\mathrm{q}, J_{\mathrm{C}-\mathrm{F}}=256.4 \mathrm{~Hz}\right)$, 118.0, 67.8, 67.4, 67.1, 46.6. Anal. Calcd for $\mathrm{C}_{19} \mathrm{H}_{15} \mathrm{~F}_{3} \mathrm{~N}_{4} \mathrm{O}_{5}$ : C, 52.30; H, 3.47; N, 12.84. Found: C, 52.02; H, 3.43; N, 12.72 .

Compounds of Table 3 . The following section details the syntheses of compounds $R-122, R-136, R-140, R-147, R-151$, and $R$ 155 of Table 3 , via representative procedures and key intermediates, as described in Schemes 4 and 5. For the syntheses of the other compounds in Table 3, see the Supporting Information.

Synthesis of R-122 (Scheme 4A). (6R)-2-Nitro-6-[(prop-2-yn-1yl)oxy]-6,7-dihydro-5H-imidazo[2,1-b][1,3]oxazine (121). A solution of alcohol $56^{32}(430 \mathrm{mg}, 2.32 \mathrm{mmol})$ and (3-bromoprop-1-yn-1yl)(tert-butyl)dimethylsilane ${ }^{41}(\mathbf{1 2 0})(900 \mathrm{mg}, 3.86 \mathrm{mmol})$ in anhydrous DMF $(6 \mathrm{~mL})$ under $\mathrm{N}_{2}$ was treated with $60 \% \mathrm{NaH}(148$ $\mathrm{mg}, 3.70 \mathrm{mmol}$ ), and the mixture was stirred at $20^{\circ} \mathrm{C}$ for $15 \mathrm{~min}$. The resulting mixture was diluted with water $(100 \mathrm{~mL})$, and the precipitate was collected by filtration, washed with water and petroleum ether, and dried. This solid was then redissolved in THF $(10 \mathrm{~mL})$; TBAF $(6.0$ $\mathrm{mL}$ of a $1 \mathrm{M}$ solution in THF, $6.0 \mathrm{mmol}$ ) was added, and the mixture was stirred at $20^{\circ} \mathrm{C}$ for $30 \mathrm{~min}$. The resulting mixture was diluted with EtOAc $(100 \mathrm{~mL})$ and washed with water $(2 \times 100 \mathrm{~mL})$ and brine $(100 \mathrm{~mL})$, back-extracting with EtOAc $(100 \mathrm{~mL})$. The combined extracts were dried $\left(\mathrm{Na}_{2} \mathrm{SO}_{4}\right)$ and then evaporated to dryness under reduced pressure, and the residue was chromatographed on silica gel. Elution with $50 \% \mathrm{EtOAc} /$ petroleum ether first gave forerunners, and then further elution with EtOAc gave $121(312 \mathrm{mg}, 60 \%)$ as a cream solid: $\mathrm{mp}\left(\mathrm{Et}_{2} \mathrm{O} /\right.$ pentane $) 81-83{ }^{\circ} \mathrm{C} ;{ }^{1} \mathrm{H}$ NMR $\left(\mathrm{CDCl}_{3}\right) \delta 7.42$ (s, 1 H), 4.63 (ddd, $J=12.5,3.8,2.1 \mathrm{~Hz}, 1 \mathrm{H}), 4.42-4.33(\mathrm{~m}, 3 \mathrm{H}), 4.32$ $(\mathrm{dd}, J=16.5,2.5 \mathrm{~Hz}, 1 \mathrm{H}), 4.25(\mathrm{dd}, J=13.1,3.6 \mathrm{~Hz}, 1 \mathrm{H}), 4.18(\mathrm{dt}, J$ $=13.0,2.5 \mathrm{~Hz}, 1 \mathrm{H}), 2.56(\mathrm{t}, J=2.4 \mathrm{~Hz}, 1 \mathrm{H})$; HRESIMS calcd for $\mathrm{C}_{9} \mathrm{H}_{9} \mathrm{~N}_{3} \mathrm{NaO}_{4} m / z[\mathrm{M}+\mathrm{Na}]^{+} 246.0485$, found 246.0484 .

Procedure J: (6R)-2-Nitro-6-(\{3-[4-(trifluoromethoxy)phenyl]prop2-yn-1-yl\}oxy)-6,7-dihydro-5H-imidazo[2,1-b][1,3]oxazine (R-122). A mixture of alkyne 121 (108 mg, $0.484 \mathrm{mmol}$ ), 1-iodo-4(trifluoromethoxy)benzene $(167 \mathrm{mg}, 0.580 \mathrm{mmol})$, and $\mathrm{CuI}(2 \mathrm{mg}$, 
$0.01 \mathrm{mmol})$ in triethylamine $(5 \mathrm{~mL})$ and DMF $(5 \mathrm{~mL})$ was purged with $\mathrm{N}_{2} . \mathrm{Pd}\left(\mathrm{PPh}_{3}\right)_{2} \mathrm{Cl}_{2}(7 \mathrm{mg}, 0.01 \mathrm{mmol})$ was added, and the mixture was stirred at $70{ }^{\circ} \mathrm{C}$ for 15 min under $\mathrm{N}_{2}$ and then cooled, diluted with water $(100 \mathrm{~mL})$, and extracted with EtOAc $(2 \times 100 \mathrm{~mL})$. The extracts were combined and evaporated to dryness under reduced pressure, and the residue was chromatographed on silica gel. Elution with $50 \% \mathrm{EtOAc} /$ petroleum ether first gave forerunners, and then further elution with EtOAc gave R-122 (109 mg, 59\%) as a cream solid: $\mathrm{mp} 150-152{ }^{\circ} \mathrm{C}$; ${ }^{1} \mathrm{H}$ NMR $\left[\left(\mathrm{CD}_{3}\right)_{2} \mathrm{SO}\right] \delta 8.05(\mathrm{~s}, 1 \mathrm{H}), 7.61$ $($ br d, $J=8.9 \mathrm{~Hz}, 2 \mathrm{H}), 7.39(\mathrm{br} \mathrm{d}, J=8.9 \mathrm{~Hz}, 2 \mathrm{H}), 4.66(\mathrm{dt}, J=12.1$, $2.4 \mathrm{~Hz}, 1 \mathrm{H}), 4.59(\mathrm{~s}, 2 \mathrm{H}), 4.49(\mathrm{br} \mathrm{d}, J=12.0 \mathrm{~Hz}, 1 \mathrm{H}), 4.41-4.37$ $(\mathrm{m}, 1 \mathrm{H}), 4.31(\mathrm{dt}, J=13.6,2.0 \mathrm{~Hz}, 1 \mathrm{H}), 4.25(\mathrm{dd}, J=13.6,3.2 \mathrm{~Hz}, 1$ $\mathrm{H}) ;{ }^{13} \mathrm{C}$ NMR $\left[\left(\mathrm{CD}_{3}\right)_{2} \mathrm{SO}\right] \delta 148.3\left(\mathrm{q}, J_{\mathrm{C}-\mathrm{F}}=1.5 \mathrm{~Hz}\right), 147.0,142.1$, $133.6(2 \mathrm{C}), 121.3(2 \mathrm{C}), 121.0,119.9\left(\mathrm{q}, J_{\mathrm{C}-\mathrm{F}}=257.1 \mathrm{~Hz}\right), 118.0$, 86.5, 84.7, 67.8, 66.2, 56.2, 46.6. Anal. Calcd for $\mathrm{C}_{16} \mathrm{H}_{12} \mathrm{~F}_{3} \mathrm{~N}_{3} \mathrm{O}_{5}$ : C, 50.14; H, 3.16; N, 10.96. Found: C, 50.19; H, 3.08; N, 10.85 .

Synthesis of R-136 (Scheme 4D). (6R)-2-Nitro-6-\{[5(trifluoromethyl)pyridin-2-yl]oxy\}-6,7-dihydro-5H-imidazo[2,1-b]$[1,3]$ oxazine (R-136). A solution of alcohol $56^{32}(1.00 \mathrm{~g}, 5.40 \mathrm{mmol})$ and 2-fluoro-5-(trifluoromethyl)pyridine $(265 \mathrm{mg}, 1.61 \mathrm{mmol})$ in anhydrous DMF $(20 \mathrm{~mL})$ under $\mathrm{N}_{2}$ at $-10{ }^{\circ} \mathrm{C}$ was treated with $60 \%$ $\mathrm{NaH}$ (275 $\mathrm{mg}, 6.88 \mathrm{mmol}$ ), then quickly degassed, and resealed under $\mathrm{N}_{2}$. Then 2-fluoro-5-(trifluoromethyl)pyridine $(1.32 \mathrm{~g}, 8.00 \mathrm{mmol})$ was added, and the mixture was stirred at -10 to $0{ }^{\circ} \mathrm{C}$ for $1 \mathrm{~h}$. Additional 2-fluoro-5-(trifluoromethyl)pyridine $(450 \mathrm{mg}, 2.73 \mathrm{mmol}$ ) was added, and the mixture was stirred at $20{ }^{\circ} \mathrm{C}$ for $130 \mathrm{~min}$. The resulting mixture was cooled to $-78{ }^{\circ} \mathrm{C}\left(\mathrm{CO}_{2}\right.$ /acetone $)$, the reaction quenched with ice/aqueous $\mathrm{NaHCO}_{3}(20 \mathrm{~mL})$, and then the mixture added to brine $(100 \mathrm{~mL})$ and extracted with $\mathrm{CH}_{2} \mathrm{Cl}_{2}(7 \times 100 \mathrm{~mL})$. The combined extracts were evaporated to dryness under reduced pressure (at $30{ }^{\circ} \mathrm{C}$ ), and the residue was chromatographed on silica gel. Elution with $\mathrm{CH}_{2} \mathrm{Cl}_{2}$ first gave forerunners, and then further elution with $0-2 \% \mathrm{EtOAc} / \mathrm{CH}_{2} \mathrm{Cl}_{2}$ gave the product, which was triturated in $\mathrm{Et}_{2} \mathrm{O}(10 \mathrm{~mL})$ and diluted with pentane $(90 \mathrm{~mL})$ to give $R-136$ (1.66 g, 93\%) as a cream solid: $\mathrm{mp} 140-141{ }^{\circ} \mathrm{C}$; ${ }^{1} \mathrm{H}$ NMR $\left[\left(\mathrm{CD}_{3}\right)_{2} \mathrm{SO}\right] \delta 8.68-8.64(\mathrm{~m}, 1 \mathrm{H}), 8.13(\mathrm{br} \mathrm{dd}, J=8.7,2.6 \mathrm{~Hz}, 1 \mathrm{H})$, $8.05(\mathrm{~s}, 1 \mathrm{H}), 7.09(\mathrm{~d}, J=8.7 \mathrm{~Hz}, 1 \mathrm{H}), 5.86-5.79(\mathrm{~m}, 1 \mathrm{H}), 4.75(\mathrm{dt}, J$ $=12.3,2.1 \mathrm{~Hz}, 1 \mathrm{H}), 4.71(\mathrm{br} \mathrm{d}, J=12.2 \mathrm{~Hz}, 1 \mathrm{H}), 4.49(\mathrm{dd}, J=14.0$, $3.4 \mathrm{~Hz}, 1 \mathrm{H}), 4.42(\mathrm{br} \mathrm{d}, J=14.1 \mathrm{~Hz}, 1 \mathrm{H}) ;{ }^{13} \mathrm{C} \mathrm{NMR}\left[\left(\mathrm{CD}_{3}\right)_{2} \mathrm{SO}\right] \delta$ $163.9,146.9,144.8\left(\mathrm{q}, J_{\mathrm{C}-\mathrm{F}}=4.5 \mathrm{~Hz}\right), 142.1,137.2\left(\mathrm{q}, J_{\mathrm{C}-\mathrm{F}}=3.1 \mathrm{~Hz}\right)$, $124.0\left(\mathrm{q}, J_{\mathrm{C}-\mathrm{F}}=271.5 \mathrm{~Hz}\right), 119.7\left(\mathrm{q}, J_{\mathrm{C}-\mathrm{F}}=32.6 \mathrm{~Hz}\right), 118.0,112.1$, 68.3, 64.3, 46.6. Anal. Calcd for $\mathrm{C}_{12} \mathrm{H}_{9} \mathrm{~F}_{3} \mathrm{~N}_{4} \mathrm{O}_{4}$ : C, 43.65; H, 2.75; N, 16.97. Found: C, $43.80 ; \mathrm{H}, 2.69$; N, 17.18 .

Synthesis of R-140 (Scheme 4E). Procedure K: (6R)-2-Nitro-6,7dihydro-5H-imidazo[2,1-b][1,3]oxazin-6-yl [4-(Trifluoromethoxy)phenyl]carbamate (R-140). 1-Isocyanato-4-(trifluoromethoxy)benzene $(70 \mu \mathrm{L}, 0.464 \mathrm{mmol})$ and $\mathrm{CuCl}(3.3 \mathrm{mg}, 0.033 \mathrm{mmol})$ were successively added to a solution of alcohol $56^{32}(54.2 \mathrm{mg}, 0.293$ $\mathrm{mmol})$ in anhydrous DMF $(0.7 \mathrm{~mL})$ under $\mathrm{N}_{2}$. The mixture was briefly degassed and resealed under $\mathrm{N}_{2}$ and then stirred at $20^{\circ} \mathrm{C}$ for 33 h. The resulting solution was treated with ice/water $(5 \mathrm{~mL})$, added to brine $(50 \mathrm{~mL})$, and extracted with $\mathrm{CH}_{2} \mathrm{Cl}_{2}(6 \times 50 \mathrm{~mL})$. The combined extracts were evaporated to dryness under reduced pressure (at $30^{\circ} \mathrm{C}$ ), and the residue was chromatographed on silica gel. Elution with $0-3 \% \mathrm{EtOAc} / \mathrm{CH}_{2} \mathrm{Cl}_{2}$ first gave forerunners, and then further elution with $3-4 \% \mathrm{EtOAc} / \mathrm{CH}_{2} \mathrm{Cl}_{2}$ gave $\mathrm{R}-\mathbf{1 4 0}(108 \mathrm{mg}, 95 \%)$ as a cream solid: $\mathrm{mp}\left(\mathrm{CH}_{2} \mathrm{Cl}_{2} /\right.$ hexane $)$ 203-205 ${ }^{\circ} \mathrm{C} ;{ }^{1} \mathrm{H}$ NMR $\left[\left(\mathrm{CD}_{3}\right)_{2} \mathrm{SO}\right] \delta 10.11($ br s, $1 \mathrm{H}), 8.09(\mathrm{~s}, 1 \mathrm{H}), 7.56($ br d, $J=8.8$ $\mathrm{Hz}, 2 \mathrm{H}), 7.30(\mathrm{br} \mathrm{d}, J=8.6 \mathrm{~Hz}, 2 \mathrm{H}), 5.47-5.41(\mathrm{~m}, 1 \mathrm{H}), 4.69-4.60$ $(\mathrm{m}, 2 \mathrm{H}), 4.43(\mathrm{dd}, J=14.0,3.4 \mathrm{~Hz}, 1 \mathrm{H}), 4.33$ (br dd, $J=14.0,1.1$ $\mathrm{Hz}, 1 \mathrm{H}) ;{ }^{13} \mathrm{C}$ NMR $\left[\left(\mathrm{CD}_{3}\right)_{2} \mathrm{SO}\right] \delta 152.2,146.8,143.3\left(\mathrm{q}, J_{\mathrm{C}-\mathrm{F}}=1.6\right.$ $\mathrm{Hz}), 142.1,137.9,121.7(2 \mathrm{C}), 120.1\left(\mathrm{q}, J_{\mathrm{C}-\mathrm{F}}=255.5 \mathrm{~Hz}\right), 119.6(2$ C), 117.9, 68.5, 62.9, 47.0. Anal. Calcd for $\mathrm{C}_{14} \mathrm{H}_{11} \mathrm{~F}_{3} \mathrm{~N}_{4} \mathrm{O}_{6}$ : C, 43.31; $\mathrm{H}, 2.86$; N, 14.43. Found: C, 43.43; H, 2.73; N, 14.35 .

Syntheses of $R-147$ and $R-151$ (Scheme 5A). (6S)-2-Nitro-6,7dihydro-5H-imidazo[2,1-b][1,3]oxazin-6-yl 4-Methylbenzene-1-sulfonate (144). A mixture of (6S)-2-nitro-6,7-dihydro-5H-imidazo[2,1b] $[1,3]$ oxazin-6-ol $^{32}(65)(5.00 \mathrm{~g}, 27.0 \mathrm{mmol})$ and 4-methylbenzene1-sulfonyl chloride $(10.4 \mathrm{~g}, 54.6 \mathrm{mmol})$ in anhydrous pyridine $(25$ $\mathrm{mL}$ ) was stirred at $49{ }^{\circ} \mathrm{C}$ for $17 \mathrm{~h}$. The cooled solution was added to crushed ice $(\sim 0.7 \mathrm{~L})$, and the resulting precipitate was collected by filtration, washing with water and petroleum ether, to give $144(8.62 \mathrm{~g}$, 94\%) as a cream solid: $\mathrm{mp} 238-242{ }^{\circ} \mathrm{C} \mathrm{dec} ;{ }^{1} \mathrm{H}$ NMR $\left[\left(\mathrm{CD}_{3}\right)_{2} \mathrm{SO}\right] \delta$ $8.01(\mathrm{~s}, 1 \mathrm{H}), 7.85(\mathrm{br} \mathrm{d}, J=8.3 \mathrm{~Hz}, 2 \mathrm{H}), 7.51(\mathrm{br} \mathrm{d}, J=8.0 \mathrm{~Hz}, 2 \mathrm{H})$, $5.41-5.34(\mathrm{~m}, 1 \mathrm{H}), 4.56(\mathrm{br} \mathrm{d}, J=12.3 \mathrm{~Hz}, 1 \mathrm{H}), 4.42(\mathrm{dt}, J=12.6$, $2.6 \mathrm{~Hz}, 1 \mathrm{H}), 4.35(\mathrm{dd}, J=14.3,3.2 \mathrm{~Hz}, 1 \mathrm{H}), 4.20(\mathrm{dt}, J=14.4,1.9$ $\mathrm{Hz}, 1 \mathrm{H}), 2.44(\mathrm{~s}, 3 \mathrm{H})$. Anal. Calcd for $\mathrm{C}_{13} \mathrm{H}_{13} \mathrm{~N}_{3} \mathrm{O}_{6} \mathrm{~S}$ : C, 46.02; $\mathrm{H}$, 3.86; N, 12.38. Found: C, 46.25; H, 3.74; N, 12.47 .

(6R)-6-Azido-2-nitro-6,7-dihydro-5H-imidazo[2,1-b][1,3]oxazine (145). A mixture of tosylate $144(8.61 \mathrm{~g}, 25.4 \mathrm{mmol})$ and sodium azide $(2.48 \mathrm{~g}, 38.1 \mathrm{mmol})$ in anhydrous DMSO $(60 \mathrm{~mL})$ was stirred at 64 ${ }^{\circ} \mathrm{C}$ for $84 \mathrm{~h}$. The resulting cooled solution was added to water $(250$ $\mathrm{mL})$ and extracted with EtOAc $(5 \times 250 \mathrm{~mL})$; the initially formed emulsion required filtration to remove a dark brown tar. The combined extracts were evaporated to dryness under reduced pressure (at $50{ }^{\circ} \mathrm{C}$ ), and the residue was triturated in EtOAc $(45 \mathrm{~mL})$, diluted with petroleum ether $(15 \mathrm{~mL})$, and filtered to give $145(3.54 \mathrm{~g}, 66 \%)$ as a brown solid. The filtrate was evaporated to dryness under reduced pressure, and the residue was chromatographed on silica gel. Elution with $0-0.33 \% \mathrm{MeOH} / \mathrm{CH}_{2} \mathrm{Cl}_{2}$ first gave forerunners, and then further elution with $0.33-0.5 \% \mathrm{MeOH} / \mathrm{CH}_{2} \mathrm{Cl}_{2}$ gave crude 145 , which was chromatographed again on silica gel. Elution with $\mathrm{Et}_{2} \mathrm{O}$ first gave forerunners, and then further elution with $0-0.33 \% \mathrm{MeOH} / \mathrm{CH}_{2} \mathrm{Cl}_{2}$ gave additional 145 (363 $\mathrm{mg}, 7 \%$ ) as a cream solid: $\mathrm{mp}$ (EtOAc/ hexane) $154-156{ }^{\circ} \mathrm{C} ;{ }^{1} \mathrm{H}$ NMR $\left[\left(\mathrm{CD}_{3}\right)_{2} \mathrm{SO}\right] \delta 8.08(\mathrm{~s}, 1 \mathrm{H}), 4.67-$ $4.61(\mathrm{~m}, 1 \mathrm{H}), 4.59$ (br dd, $J=11.9,1.4 \mathrm{~Hz}, 1 \mathrm{H}), 4.55$ (ddd, $J=11.9$, $2.8,2.0 \mathrm{~Hz}, 1 \mathrm{H}), 4.32(\mathrm{dd}, J=13.5,3.8 \mathrm{~Hz}, 1 \mathrm{H}), 4.17(\mathrm{br} \mathrm{dt}, J=13.5$, $1.8 \mathrm{~Hz}, 1 \mathrm{H})$; $[\alpha]^{25} 92.6$ (c 1.004, DMF). Anal. Calcd for $\mathrm{C}_{6} \mathrm{H}_{6} \mathrm{~N}_{6} \mathrm{O}_{3}$ : C, 34.29; H, 2.88; N, 39.99. Found: C, 34.57; H, 2.66; N, 40.03.

(6R)-2-Nitro-6,7-dihydro-5H-imidazo[2,1-b][1,3]oxazin-6-amine Hydrochloride (146). Propane-1,3-dithiol $(9.2 \mathrm{~mL}, 91.6 \mathrm{mmol})$ was added to a mixture of azide $145(3.83 \mathrm{~g}, 18.2 \mathrm{mmol})$ and triethylamine $(12.8 \mathrm{~mL}, 91.8 \mathrm{mmol})$ in anhydrous $\mathrm{MeOH}(75 \mathrm{~mL})$ under $\mathrm{N}_{2}$. After being stirred at $20{ }^{\circ} \mathrm{C}$ for $30 \mathrm{~min}$, the mixture was evaporated to dryness under reduced pressure (at $30{ }^{\circ} \mathrm{C}$ ), and the residue was chromatographed on silica gel. Elution with $0-3.5 \% \mathrm{MeOH} / \mathrm{CH}_{2} \mathrm{Cl}_{2}$ first gave forerunners, and then further elution with $5-8 \% \mathrm{MeOH}$ / $\mathrm{CH}_{2} \mathrm{Cl}_{2}$ gave the product free base $(2.25 \mathrm{~g}, 67 \%)$ as a yellow solid, which was used directly: ${ }^{1} \mathrm{H}$ NMR $\left[\left(\mathrm{CD}_{3}\right)_{2} \mathrm{SO}\right] \delta 8.06(\mathrm{~s}, 1 \mathrm{H}), 4.35$ (br dd, $J=10.8,2.1 \mathrm{~Hz}, 1 \mathrm{H}), 4.19-4.10(\mathrm{~m}, 2 \mathrm{H}), 3.76$ (ddd, $J=12.4$, $5.5,1.0 \mathrm{~Hz}, 1 \mathrm{H}), 3.54-3.13(\mathrm{~m}, 3 \mathrm{H})$; APCI MS $m / z 185[\mathrm{M}+\mathrm{H}]^{+}$. This free base was dissolved in $\mathrm{MeOH}(10 \mathrm{~mL})$ and dioxane $(10 \mathrm{~mL})$ and treated with a solution of $\mathrm{HCl}$ in dioxane $(4.6 \mathrm{~mL}$ of a $4 \mathrm{M}$ solution, $18.4 \mathrm{mmol}$ ), and then the mixture was diluted with $\mathrm{Et}_{2} \mathrm{O}$ $(100 \mathrm{~mL})$. The resulting oily precipitate was triturated to give $\mathbf{1 4 6}$ $(2.29 \mathrm{~g}, 57 \%)$ as a bright yellow powder: $\mathrm{mp} 208{ }^{\circ} \mathrm{C} \mathrm{dec} ;{ }^{1} \mathrm{H}$ NMR $\left[\left(\mathrm{CD}_{3}\right)_{2} \mathrm{SO}\right] \delta 8.73(\mathrm{br} \mathrm{s}, 3 \mathrm{H}), 8.18(\mathrm{~s}, 1 \mathrm{H}), 4.63(\mathrm{dd}, J=12.2,2.0$ $\mathrm{Hz}, 1 \mathrm{H}), 4.59(\mathrm{dt}, J=12.1,2.2 \mathrm{~Hz}, 1 \mathrm{H}), 4.41(\mathrm{dd}, J=14.0,5.0 \mathrm{~Hz}, 1$ $\mathrm{H}), 4.21(\mathrm{br} \mathrm{d}, J=14.1 \mathrm{~Hz}, 1 \mathrm{H}), 4.16-4.07(\mathrm{~m}, 1 \mathrm{H})$; $[\alpha]_{\mathrm{D}}^{27} 74.7(c$ 1.004, $\left.\mathrm{H}_{2} \mathrm{O}\right)$; HRESIMS calcd for $\mathrm{C}_{6} \mathrm{H}_{9} \mathrm{~N}_{4} \mathrm{O}_{3} \mathrm{~m} / z\left[\mathrm{M}-\mathrm{Cl}^{-}\right]^{+}$ 185.0669 , found 185.0673 .

Procedure L: (6R)-2-Nitro-N-[4-(trifluoromethoxy)benzyl]-6,7-dihydro-5H-imidazo $[2,1-b][1,3]$ oxazin-6-amine $(R-147)$. 4(Trifluoromethoxy)benzaldehyde $(130 \mu \mathrm{L}, 0.910 \mathrm{mmol})$ was added to a mixture of amine salt $146(120 \mathrm{mg}, 0.544 \mathrm{mmol})$ and $\mathrm{AcOH}(65$ $\mu \mathrm{L}, 1.14 \mathrm{mmol})$ in anhydrous DMF $(5 \mathrm{~mL})$ under $\mathrm{N}_{2}$. The mixture was stirred at $20{ }^{\circ} \mathrm{C}$ for $15 \mathrm{~min}$ and then cooled to $0{ }^{\circ} \mathrm{C}$. Sodium cyanoborohydride ( $73 \mathrm{mg}, 1.16 \mathrm{mmol}$ ) was added, and the mixture was quickly degassed and resealed under $\mathrm{N}_{2}$ and then stirred at $20{ }^{\circ} \mathrm{C}$ for $7 \mathrm{~h}$. The resulting mixture was cooled to $-78{ }^{\circ} \mathrm{C}\left(\mathrm{CO}_{2} /\right.$ acetone $)$, the reaction quenched with ice/aqueous $\mathrm{Na}_{2} \mathrm{CO}_{3}(10 \mathrm{~mL})$, and the mixture added to brine $(40 \mathrm{~mL})$ and extracted with $\mathrm{CH}_{2} \mathrm{Cl}_{2}(6 \times 50$ $\mathrm{mL})$. The combined extracts were evaporated to dryness under reduced pressure $\left(\right.$ at $\left.30{ }^{\circ} \mathrm{C}\right)$, and the residue was chromatographed on silica gel. Elution with $0-0.25 \% \mathrm{MeOH} / \mathrm{CH}_{2} \mathrm{Cl}_{2}$ first gave forerunners, and then further elution with $0.33-0.5 \% \mathrm{MeOH} / \mathrm{CH}_{2} \mathrm{Cl}_{2}$ gave $\mathrm{R}-147$ (150 mg, 77\%) as a cream solid: $\mathrm{mp}\left(\mathrm{Et}_{2} \mathrm{O} / \mathrm{CH}_{2} \mathrm{Cl}_{2} /\right.$ hexane $) 120-121$ ${ }^{\circ} \mathrm{C} ;{ }^{1} \mathrm{H}$ NMR $\left[\left(\mathrm{CD}_{3}\right)_{2} \mathrm{SO}\right] \delta 8.01(\mathrm{~s}, 1 \mathrm{H}), 7.45(\mathrm{br} \mathrm{d}, J=8.6 \mathrm{~Hz}, 2$ $\mathrm{H}), 7.30(\mathrm{br} \mathrm{d}, J=8.0 \mathrm{~Hz}, 2 \mathrm{H}), 4.43(\mathrm{dd}, J=11.2,2.2 \mathrm{~Hz}, 1 \mathrm{H}), 4.38$ (ddd, $J=11.3,4.3,1.3 \mathrm{~Hz}, 1 \mathrm{H}$ ), $4.16(\mathrm{dd}, J=12.7,4.1 \mathrm{~Hz}, 1 \mathrm{H}$ ), 3.99 
(dd, $J=12.7,2.7 \mathrm{~Hz}, 1 \mathrm{H}$ ), 3.81 (br s, $2 \mathrm{H}$ ), 3.29-3.19 (m, $1 \mathrm{H}), 2.83$ (br s, $1 \mathrm{H}) ;{ }^{13} \mathrm{C}$ NMR $\left[\left(\mathrm{CD}_{3}\right)_{2} \mathrm{SO}\right] \delta 147.4,147.1,142.1,139.9,129.6$ (2 C), $120.8(2 \mathrm{C}), 120.1\left(\mathrm{q}, J_{\mathrm{C}-\mathrm{F}}=255.6 \mathrm{~Hz}\right), 118.1,68.8,48.8,47.2$, 46.8. Anal. Calcd for $\mathrm{C}_{14} \mathrm{H}_{13} \mathrm{~F}_{3} \mathrm{~N}_{4} \mathrm{O}_{4}$ : C, 46.93; H, 3.66; N, 15.64 . Found: C, 47.12; H, 3.54; N, 15.89 .

Procedure $\mathrm{M}: \mathrm{N}-[(6 \mathrm{R})-2-N i t r o-6,7-d i h y d r o-5 H$-imidazo[2,1-b][1,3]oxazin-6-yl]-3-(trifluoromethoxy)benzamide (R-151). 3(Trifluoromethoxy)benzoyl chloride (205 $\mathrm{mg}, 0.913 \mathrm{mmol}$ ) was added to a solution of amine salt $146(152 \mathrm{mg}, 0.689 \mathrm{mmol})$ and DIPEA $(0.30 \mathrm{~mL}, 1.72 \mathrm{mmol})$ in anhydrous DMF $(3 \mathrm{~mL})$ under $\mathrm{N}_{2}$. The mixture was stirred at $20{ }^{\circ} \mathrm{C}$ for $10 \mathrm{~h}$ and then treated with ice/ water $(5 \mathrm{~mL})$, added to brine $(40 \mathrm{~mL})$, and extracted with $\mathrm{CH}_{2} \mathrm{Cl}_{2}(4$ $\times 50 \mathrm{~mL}$ ). The combined extracts were evaporated to dryness under reduced pressure $\left(\right.$ at $30{ }^{\circ} \mathrm{C}$ ), and the residue was chromatographed on silica gel. Elution with $0-0.5 \% \mathrm{MeOH} / \mathrm{CH}_{2} \mathrm{Cl}_{2}$ first gave forerunners, and then further elution with $0.5-0.67 \% \mathrm{MeOH} / \mathrm{CH}_{2} \mathrm{Cl}_{2}$ gave $\mathrm{R}-151$ (213 mg, 83\%) as a cream solid: $\mathrm{mp}\left(\mathrm{Et}_{2} \mathrm{O} /\right.$ pentane $) 98-101{ }^{\circ} \mathrm{C}$ dec; ${ }^{1} \mathrm{H}$ NMR $\left[\left(\mathrm{CD}_{3}\right)_{2} \mathrm{SO}\right] \delta 9.01(\mathrm{br} \mathrm{d}, J=6.4 \mathrm{~Hz}, 1 \mathrm{H}), 8.13(\mathrm{~s}, 1 \mathrm{H})$, $7.90(\mathrm{dt}, J=7.6,1.3 \mathrm{~Hz}, 1 \mathrm{H}), 7.83-7.78(\mathrm{~m}, 1 \mathrm{H}), 7.63$ (dd, $J=8.0$, $7.7 \mathrm{~Hz}, 1 \mathrm{H}), 7.60-7.55(\mathrm{~m}, 1 \mathrm{H}), 4.69-4.61(\mathrm{~m}, 1 \mathrm{H}), 4.57(\mathrm{dd}, J=$ $11.2,2.3 \mathrm{~Hz}, 1 \mathrm{H}), 4.51(\mathrm{ddd}, J=11.3,4.1,1.4 \mathrm{~Hz}, 1 \mathrm{H}), 4.41(\mathrm{dd}, J=$ 13.0, 5.1 Hz, $1 \mathrm{H}), 4.17$ (ddd, $J=13.1,3.3,1.2 \mathrm{~Hz}, 1 \mathrm{H}) ;{ }^{13} \mathrm{C} \mathrm{NMR}$ $\left[\left(\mathrm{CD}_{3}\right)_{2} \mathrm{SO}\right] \delta 165.4,148.2,147.2,142.2,135.9,130.5,126.8,124.1$, 120.1, $120.0\left(\mathrm{q}, J_{\mathrm{C}-\mathrm{F}}=256.8 \mathrm{~Hz}\right), 118.4,68.5,46.5$, 41.6. Anal. Calcd for $\mathrm{C}_{14} \mathrm{H}_{11} \mathrm{~F}_{3} \mathrm{~N}_{4} \mathrm{O}_{5}$ : C, 45.17; H, 2.98; N, 15.05. Found: C, 45.37; H, $2.98 ; \mathrm{N}, 15.07$.

Synthesis of R-155 (Scheme 5B). Procedure $N$ : N-[(6R)-2-Nitro6,7-dihydro-5H-imidazo $[2,1-b][1,3]$ oxazin-6-yl]- $N^{\prime}-[2-$ (trifluoromethoxy)phenyl]urea $(R-155)$. 1-Isocyanato-2(trifluoromethoxy)benzene $(80 \mu \mathrm{L}, 0.532 \mathrm{mmol})$ was added to a solution of amine salt $146(80.2 \mathrm{mg}, 0.364 \mathrm{mmol})$, DIPEA $(0.155 \mathrm{~mL}$, $0.890 \mathrm{mmol}$ ), and dibutyltin diacetate $(9.3 \mathrm{mg}, 0.026 \mathrm{mmol})$ in anhydrous DMF $(2 \mathrm{~mL})$ under $\mathrm{N}_{2}$. The mixture was stirred at $20{ }^{\circ} \mathrm{C}$ for $18 \mathrm{~h}$ and then treated with ice/water $(5 \mathrm{~mL})$, added to brine $(40$ $\mathrm{mL})$, and extracted with $\mathrm{CH}_{2} \mathrm{Cl}_{2}(5 \times 50 \mathrm{~mL})$. The combined extracts were evaporated to dryness under reduced pressure (at $30{ }^{\circ} \mathrm{C}$ ), and the residue was chromatographed on silica gel. Elution with $0-0.5 \%$ $\mathrm{MeOH} / \mathrm{CH}_{2} \mathrm{Cl}_{2}$ first gave forerunners, and then further elution with $0.75-1 \% \mathrm{MeOH} / \mathrm{CH}_{2} \mathrm{Cl}_{2}$ gave $\mathrm{R}-\mathbf{1 5 5}(118 \mathrm{mg}, 84 \%)$ as a cream solid: $\mathrm{mp}\left(\mathrm{MeOH} / \mathrm{CH}_{2} \mathrm{Cl}_{2} /\right.$ hexane) $221-225{ }^{\circ} \mathrm{C} \mathrm{dec} ;{ }^{1} \mathrm{H}$ NMR $\left[\left(\mathrm{CD}_{3}\right)_{2} \mathrm{SO}\right] \delta 8.27(\mathrm{dd}, J=8.5,1.6 \mathrm{~Hz}, 1 \mathrm{H}), 8.26($ br s, $1 \mathrm{H})$, $8.11(\mathrm{~s}, 1 \mathrm{H}), 7.58(\mathrm{br} \mathrm{d}, J=7.0 \mathrm{~Hz}, 1 \mathrm{H}), 7.35-7.25(\mathrm{~m}, 2 \mathrm{H}), 7.03$ (ddd, $J=8.0,7.7,1.6 \mathrm{~Hz}, 1 \mathrm{H}), 4.59$ (dd, $J=11.3,1.8 \mathrm{~Hz}, 1 \mathrm{H}), 4.48$ (ddd, $J=11.3,3.0,2.2 \mathrm{~Hz}, 1 \mathrm{H}), 4.44-4.36(\mathrm{~m}, 1 \mathrm{H}), 4.31(\mathrm{dd}, J=$ $12.8,4.1 \mathrm{~Hz}, 1 \mathrm{H}), 4.12(\mathrm{dt}, J=12.9,2.1 \mathrm{~Hz}, 1 \mathrm{H}) ;{ }^{13} \mathrm{C}$ NMR $\left[\left(\mathrm{CD}_{3}\right)_{2} \mathrm{SO}\right] \delta 154.2,147.0,142.1,136.7,132.5,127.8,122.1,121.1$, $120.3,120.2\left(\mathrm{q}, J_{\mathrm{C}-\mathrm{F}}=257.6 \mathrm{~Hz}\right), 118.4,69.7,47.8,41.0$. Anal. Calcd for $\mathrm{C}_{14} \mathrm{H}_{12} \mathrm{~F}_{3} \mathrm{~N}_{5} \mathrm{O}_{5}$ : C, 43.42; H, 3.12; N, 18.08. Found: C, 43.48; H, 2.99; N, 18.03 .

Compounds of Table 4. The following section details the synthesis of compound R-168 of Table 4, via representative procedures and key intermediates, as described in Scheme 5. For the syntheses of the other compounds in Table 4, see the Supporting Information.

Synthesis of R-168 (Scheme 5D). (2R)-2-[(6-Bromopyridin-3yl)oxy]-3-[(4-methoxybenzyl)oxy]propan-1-ol (163). Reaction of 2bromo-5-(\{(2S)-1-[(4-methoxybenzyl)oxy]-3-[(triisopropylsilyl)oxy]propan-2-yl\}oxy)pyridine ${ }^{42}$ (162) with TBAF (1.2 equiv), using procedure $\mathrm{C}$ for $13 \mathrm{~h}$ (extracting the product four times with EtOAc), followed by chromatography of the product on silica gel, eluting with 0-30\% $\mathrm{Et}_{2} \mathrm{O}$ /petroleum ether and $\mathrm{CH}_{2} \mathrm{Cl}_{2}$ (forerunners) and then with $2 \% \mathrm{MeOH} / \mathrm{CH}_{2} \mathrm{Cl}_{2}$, gave $163(94 \%)$ as a white solid: $\mathrm{mp}$ $\left(\mathrm{CH}_{2} \mathrm{Cl}_{2} /\right.$ pentane $) 78-80{ }^{\circ} \mathrm{C} ;{ }^{1} \mathrm{H}$ NMR $\left(\mathrm{CDCl}_{3}\right) \delta 8.12(\mathrm{br} \mathrm{d}, J=3.0$ $\mathrm{Hz}, 1 \mathrm{H}), 7.35($ br d, $J=8.7 \mathrm{~Hz}, 1 \mathrm{H}), 7.20(\mathrm{br} \mathrm{d}, J=8.8 \mathrm{~Hz}, 2 \mathrm{H})$, $7.18(\mathrm{dd}, J=8.7,3.1 \mathrm{~Hz}, 1 \mathrm{H}), 6.87(\mathrm{br} \mathrm{d}, J=8.7 \mathrm{~Hz}, 2 \mathrm{H}), 4.51-4.42$ (m, $3 \mathrm{H}), 3.94-3.81(\mathrm{~m}, 2 \mathrm{H}), 3.81(\mathrm{~s}, 3 \mathrm{H}), 3.67(\mathrm{~d}, J=5.1 \mathrm{~Hz}, 2 \mathrm{H})$, $1.95(\mathrm{t}, J=6.4 \mathrm{~Hz}, 1 \mathrm{H}) ;[\alpha]^{23}{ }_{\mathrm{D}} 19.8\left(\mathrm{c} 3.024, \mathrm{CHCl}_{3}\right)$. Anal. Calcd for $\mathrm{C}_{16} \mathrm{H}_{18} \mathrm{BrNO}_{4}$ : C, 52.19; H, 4.93; N, 3.80. Found: C, 52.19; H, 4.82; $\mathrm{N}, 3.84$.
Procedure O: 2-Bromo-5-(\{(2S)-1-iodo-3-[(4-methoxybenzyl)oxy]propan-2-y/\}oxy)pyridine (164). A solution of iodine (3.74 g, 14.7 mmol $)$ in anhydrous $\mathrm{CH}_{2} \mathrm{Cl}_{2}(9 \times 10 \mathrm{~mL})$ was slowly added (dropwise over $1 \mathrm{~h}$ ) to a stirred mixture of alcohol 163 (3.97 g, 10.8 $\mathrm{mmol})$, imidazole $(1.92 \mathrm{~g}, 28.2 \mathrm{mmol})$, and triphenylphosphine $(3.70$ $\mathrm{g}, 14.1 \mathrm{mmol})$ in anhydrous $\mathrm{CH}_{2} \mathrm{Cl}_{2}(50 \mathrm{~mL})$ under $\mathrm{N}_{2}$ at $20{ }^{\circ} \mathrm{C}$ (water bath cooling). After being stirred at $20^{\circ} \mathrm{C}$ for $41 \mathrm{~h}$, the mixture was concentrated under reduced pressure $\left(\right.$ at $25{ }^{\circ} \mathrm{C}$ ), and the residual oil was added to excess petroleum ether $(100 \mathrm{~mL})$ at the top of a silica gel column ( $50 \mathrm{~g}$ in petroleum ether), rinsing on with $\mathrm{CH}_{2} \mathrm{Cl}_{2}(4 \times 5$ $\mathrm{mL}$ ). Elution with $0-10 \% \mathrm{Et}_{2} \mathrm{O} /$ petroleum ether first gave forerunners, and then further elution with $20-33 \% \mathrm{Et}_{2} \mathrm{O} /$ petroleum ether gave $164(5.17 \mathrm{~g}, 100 \%)$ as a colorless oil: ${ }^{1} \mathrm{H}$ NMR $\left(\mathrm{CDCl}_{3}\right) \delta$ $8.11(\mathrm{~d}, J=3.1 \mathrm{~Hz}, 1 \mathrm{H}), 7.36(\mathrm{~d}, J=8.7 \mathrm{~Hz}, 1 \mathrm{H}), 7.22(\mathrm{br} \mathrm{d}, J=8.7$ $\mathrm{Hz}, 2 \mathrm{H}), 7.16(\mathrm{dd}, J=8.7,3.1 \mathrm{~Hz}, 1 \mathrm{H}), 6.88(\mathrm{br} \mathrm{d}, J=8.7 \mathrm{~Hz}, 2 \mathrm{H})$, $4.51(\mathrm{~d}, J=11.6 \mathrm{~Hz}, 1 \mathrm{H}), 4.48(\mathrm{~d}, J=11.6 \mathrm{~Hz}, 1 \mathrm{H}), 4.37-4.30(\mathrm{~m}, 1$ $\mathrm{H}), 3.81(\mathrm{~s}, 3 \mathrm{H}), 3.73(\mathrm{dd}, J=10.4,5.2 \mathrm{~Hz}, 1 \mathrm{H}), 3.69(\mathrm{dd}, J=10.3$, $4.9 \mathrm{~Hz}, 1 \mathrm{H}), 3.43(\mathrm{dd}, J=10.8,5.5 \mathrm{~Hz}, 1 \mathrm{H}), 3.36(\mathrm{dd}, J=10.7,5.4$ $\mathrm{Hz}, 1 \mathrm{H})$; HRESIMS calcd for $\mathrm{C}_{16} \mathrm{H}_{18} \mathrm{BrINO}_{3} \mathrm{~m} / \mathrm{z}[\mathrm{M}+\mathrm{H}]^{+}$ 479.9490, 477.9509, found 479.9489, 477.9508.

2-Bromo-5-(\{(2R)-1-(2-bromo-4-nitro-1H-imidazol-1-yl)-3-[(4methoxybenzyl)oxy]propan-2-yl\}oxy)pyridine (165). A mixture of iodide 164 (5.17 g, $10.8 \mathrm{mmol})$, 2-bromo-4-nitro- $1 \mathrm{H}$-imidazole (129) $(2.28 \mathrm{~g}, 11.9 \mathrm{mmol})$, and powdered $\mathrm{K}_{2} \mathrm{CO}_{3}(1.79 \mathrm{~g}, 13.0 \mathrm{mmol})$ in anhydrous DMF $(26 \mathrm{~mL})$ under $\mathrm{N}_{2}$ was stirred at $88{ }^{\circ} \mathrm{C}$ for $122 \mathrm{~h}$. The resulting cooled mixture was added to aqueous $\mathrm{NaHCO}_{3}(100$ $\mathrm{mL})$ and extracted with $\mathrm{CH}_{2} \mathrm{Cl}_{2}(6 \times 100 \mathrm{~mL})$. The combined extracts were evaporated to dryness under reduced pressure (at $30{ }^{\circ} \mathrm{C}$ ), and the residue was chromatographed on silica gel. Elution with $0-30 \%$ $\mathrm{EtOAc} /$ petroleum ether first gave forerunners, and then further elution with 30-50\% EtOAc/petroleum ether gave 165 (4.44 g, 76\%) as a pale yellow solid: $\mathrm{mp}\left(\mathrm{Et}_{2} \mathrm{O} /\right.$ pentane) $86-88{ }^{\circ} \mathrm{C}$; ${ }^{1} \mathrm{H}$ NMR $\left(\mathrm{CDCl}_{3}\right) \delta 8.04(\mathrm{~d}, J=3.1 \mathrm{~Hz}, 1 \mathrm{H}), 7.83(\mathrm{~s}, 1 \mathrm{H}), 7.37(\mathrm{br} \mathrm{d}, J=8.7$ $\mathrm{Hz}, 1 \mathrm{H}), 7.24($ br d, $J=8.7 \mathrm{~Hz}, 2 \mathrm{H}), 7.02(\mathrm{dd}, J=8.7,3.2 \mathrm{~Hz}, 1 \mathrm{H})$, $6.92(\mathrm{br} \mathrm{d}, J=8.7 \mathrm{~Hz}, 2 \mathrm{H}), 4.63-4.56(\mathrm{~m}, 1 \mathrm{H}), 4.53(\mathrm{~d}, J=11.6 \mathrm{~Hz}$, $1 \mathrm{H}), 4.49(\mathrm{~d}, J=11.6 \mathrm{~Hz}, 1 \mathrm{H}), 4.46(\mathrm{dd}, J=14.8,3.8 \mathrm{~Hz}, 1 \mathrm{H}), 4.36$ $(\mathrm{dd}, J=14.7,7.1 \mathrm{~Hz}, 1 \mathrm{H}), 3.84(\mathrm{~s}, 3 \mathrm{H}), 3.62(\mathrm{dd}, J=10.5,4.1 \mathrm{~Hz}, 1$ $\mathrm{H}), 3.55(\mathrm{dd}, J=10.5,6.1 \mathrm{~Hz}, 1 \mathrm{H})$; HRESIMS calcd for $\mathrm{C}_{19} \mathrm{H}_{18} \mathrm{Br}_{2} \mathrm{~N}_{4} \mathrm{NaO}_{5} \mathrm{~m} / z[\mathrm{M}+\mathrm{Na}]^{+} 566.9500,564.9517,562.9536$, found 566.9506, 564.9522, 562.9540.

(2R)-3-(2-Bromo-4-nitro-1H-imidazol-1-yl)-2-[(6-bromopyridin-3yl)oxy]propan-1-ol (166). A mixture of PMB ether 165 (4.42 g, 8.15 $\mathrm{mmol})$ and DDQ $(1.95 \mathrm{~g}, 8.59 \mathrm{mmol})$ in $\mathrm{CH}_{2} \mathrm{Cl}_{2}(175 \mathrm{~mL})$ was stirred at $20{ }^{\circ} \mathrm{C}$ for $48 \mathrm{~h}$. Additional DDQ $(202 \mathrm{mg}, 0.89 \mathrm{mmol})$ was added, and stirring was continued at $20{ }^{\circ} \mathrm{C}$ for $50 \mathrm{~h}$. The resulting mixture was added to saturated aqueous $\mathrm{NaHCO}_{3}(200 \mathrm{~mL})$ and extracted with $\mathrm{CH}_{2} \mathrm{Cl}_{2}(4 \times 150 \mathrm{~mL})$. The extracts were sequentially washed with aqueous $\mathrm{NaHCO}_{3}(150 \mathrm{~mL})$; then the combined extracts were evaporated to dryness under reduced pressure (at $30{ }^{\circ} \mathrm{C}$ ), and the remaining oil was chromatographed on silica gel. Elution with $\mathrm{CH}_{2} \mathrm{Cl}_{2}$ first gave forerunners, and then further elution with $3 \%$ $\mathrm{MeOH} / \mathrm{CH}_{2} \mathrm{Cl}_{2}$ gave the crude product mixture $(4.09 \mathrm{~g})$ as a pale yellow foam. This material was suspended in $3: 1 \mathrm{MeOH} / \mathrm{CH}_{2} \mathrm{Cl}_{2}(200$ $\mathrm{mL})$ and treated with $p$-toluenesulfonic acid monohydrate $(0.79 \mathrm{~g}$, $4.15 \mathrm{mmol}$ ), stirring at $20{ }^{\circ} \mathrm{C}$ for $10 \mathrm{~h}$. Excess $\mathrm{NaHCO}_{3}(0.5 \mathrm{~g})$ was added, and the mixture was concentrated under reduced pressure, then diluted with aqueous $\mathrm{NaHCO}_{3}(100 \mathrm{~mL})$, and extracted with $\mathrm{CH}_{2} \mathrm{Cl}_{2}$ $(5 \times 100 \mathrm{~mL})$. The combined extracts were evaporated to dryness under reduced pressure, and the remaining oil was chromatographed on silica gel. Elution with $0-1 \% \mathrm{MeOH} / \mathrm{CH}_{2} \mathrm{Cl}_{2}$ first gave forerunners, and then further elution with $1-2 \% \mathrm{MeOH} / \mathrm{CH}_{2} \mathrm{Cl}_{2}$ gave $166(3.32 \mathrm{~g}, 96 \%)$ as a cream solid: $\mathrm{mp}\left(\mathrm{MeOH} / \mathrm{CH}_{2} \mathrm{Cl}_{2} /\right.$ hexane) $132-133{ }^{\circ} \mathrm{C} ;{ }^{1} \mathrm{H}$ NMR $\left[\left(\mathrm{CD}_{3}\right)_{2} \mathrm{SO}\right] \delta 8.58(\mathrm{~s}, 1 \mathrm{H}), 8.08(\mathrm{~d}$, $J=3.0 \mathrm{~Hz}, 1 \mathrm{H}), 7.51(\mathrm{br} \mathrm{d}, J=8.7 \mathrm{~Hz}, 1 \mathrm{H}), 7.36(\mathrm{dd}, J=8.8,3.2 \mathrm{~Hz}$, $1 \mathrm{H}), 5.25(\mathrm{brt}, J=4.7 \mathrm{~Hz}, 1 \mathrm{H}), 4.85-4.78(\mathrm{~m}, 1 \mathrm{H}), 4.44(\mathrm{dd}, J=$ 14.7, 3.9 Hz, 1 H), $4.37(\mathrm{dd}, J=14.7,7.8 \mathrm{~Hz}, 1 \mathrm{H}), 3.72-3.57(\mathrm{~m}, 2$ $\mathrm{H}) ;[\alpha]^{26} 13.3$ (c 2.032, DMF). Anal. Calcd for $\mathrm{C}_{11} \mathrm{H}_{10} \mathrm{Br}_{2} \mathrm{~N}_{4} \mathrm{O}_{4}$ : C, 31.31; H, 2.39; N, 13.28. Found: C, 31.55; H, 2.32; N, 13.34.

(6R)-6-[(6-Bromopyridin-3-yl)oxy]-2-nitro-6,7-dihydro-5Himidazo[2,1-b][1,3]oxazine (167). A solution of alcohol 166 (3.29 g, 
$7.80 \mathrm{mmol})$ in anhydrous DMF $(50 \mathrm{~mL})$ under $\mathrm{N}_{2}$ at $0{ }^{\circ} \mathrm{C}$ was treated with $60 \% \mathrm{NaH}(419 \mathrm{mg}, 10.5 \mathrm{mmol})$ and then quickly degassed and resealed under $\mathrm{N}_{2}$. The mixture was stirred at $0{ }^{\circ} \mathrm{C}$ for $45 \mathrm{~min}$ and then at $20^{\circ} \mathrm{C}$ for $160 \mathrm{~min}$, then cooled to $-78{ }^{\circ} \mathrm{C}\left(\mathrm{CO}_{2} /\right.$ acetone $)$, the reaction quenched with ice/aqueous $\mathrm{NaHCO}_{3}(20 \mathrm{~mL})$, and the mixture added to brine $(200 \mathrm{~mL})$. The resulting mixture was sequentially extracted with $\mathrm{CH}_{2} \mathrm{Cl}_{2}(150 \mathrm{~mL}), 10 \% \mathrm{MeOH} / \mathrm{CH}_{2} \mathrm{Cl}_{2}$ $(4 \times 150 \mathrm{~mL}), 20 \% \mathrm{EtOAc} / \mathrm{CH}_{2} \mathrm{Cl}_{2}(4 \times 150 \mathrm{~mL}), 25 \% \mathrm{MeOH} /$ $\mathrm{CH}_{2} \mathrm{Cl}_{2}(4 \times 150 \mathrm{~mL}), \mathrm{CH}_{2} \mathrm{Cl}_{2}(2 \times 150 \mathrm{~mL})$, and EtOAc $(150 \mathrm{~mL})$, and then the combined extracts were evaporated to dryness under reduced pressure $\left(\right.$ at $\left.30{ }^{\circ} \mathrm{C}\right)$. The crude residue was triturated in water, filtered and dried, then resuspended in warm $5 \% \mathrm{MeOH} / \mathrm{CH}_{2} \mathrm{Cl}_{2}(100$ $\mathrm{mL}$ ), cooled, and filtered to give $\mathbf{1 6 7}(1.93 \mathrm{~g}, 73 \%)$ as a pale brown solid: $\mathrm{mp} 244-247^{\circ} \mathrm{C}$; ${ }^{1} \mathrm{H}$ NMR $\left[\left(\mathrm{CD}_{3}\right)_{2} \mathrm{SO}\right] \delta 8.22(\mathrm{br} \mathrm{d}, J=3.0 \mathrm{~Hz}$, $1 \mathrm{H}), 8.05(\mathrm{~s}, 1 \mathrm{H}), 7.61(\mathrm{br} \mathrm{d}, J=8.6 \mathrm{~Hz}, 1 \mathrm{H}), 7.55(\mathrm{dd}, J=8.8,3.1$ $\mathrm{Hz}, 1 \mathrm{H}), 5.35-5.29(\mathrm{~m}, 1 \mathrm{H}), 4.69(\mathrm{dt}, J=12.4,2.1 \mathrm{~Hz}, 1 \mathrm{H}), 4.64$ $($ br d, $J=12.2 \mathrm{~Hz}, 1 \mathrm{H}), 4.40(\mathrm{dd}, J=14.0,3.0 \mathrm{~Hz}, 1 \mathrm{H}), 4.35(\mathrm{br} \mathrm{d}, J$ $=14.1 \mathrm{~Hz}, 1 \mathrm{H}) ;[\alpha]_{\mathrm{D}}^{25} 21.0(c 1.000$, DMF). Anal. Calcd for $\mathrm{C}_{11} \mathrm{H}_{9} \mathrm{BrN}_{4} \mathrm{O}_{4}: \mathrm{C}, 38.73 ; \mathrm{H}, 2.66 ; \mathrm{N}, 16.42$. Found: $\mathrm{C}, 38.67 ; \mathrm{H}, 2.54$; $\mathrm{N}, 16.40$.

The filtrate described above was evaporated to dryness under reduced pressure, and the residue was chromatographed on silica gel. Elution with $0-0.5 \% \mathrm{MeOH} / \mathrm{CH}_{2} \mathrm{Cl}_{2}$ first gave forerunners, and then further elution with $0.5-0.67 \% \mathrm{MeOH} / \mathrm{CH}_{2} \mathrm{Cl}_{2}$ gave the crude product, which was suspended in warm $10 \% \mathrm{MeOH} / \mathrm{CH}_{2} \mathrm{Cl}_{2}(15 \mathrm{~mL})$, then diluted with $\mathrm{CH}_{2} \mathrm{Cl}_{2}(20 \mathrm{~mL})$ and hexane $(50 \mathrm{~mL})$, and filtered to give additional $167(233 \mathrm{mg}, 9 \%)$.

(6R)-2-Nitro-6-(\{6-[4-(trifluoromethoxy)phenyl]pyridin-3-yl\}oxy)6,7-dihydro-5H-imidazo[2,1-b][1,3]oxazine (R-168). Reaction of bromide 167 with $\mathrm{Pd}(\mathrm{dppf}) \mathrm{Cl}_{2} \quad(0.27$ equiv) and [4(trifluoromethoxy)phenyl]boronic acid (1.9 equiv), using procedure I for $210 \mathrm{~min}$, followed by chromatography of the product on silica gel, eluting with $0-0.33 \% \mathrm{MeOH} / \mathrm{CH}_{2} \mathrm{Cl}_{2}$ (forerunners) and then with $0.5 \% \mathrm{MeOH} / \mathrm{CH}_{2} \mathrm{Cl}_{2}$, gave $\mathrm{R}-\mathbf{1 6 8}(86 \%)$ as a cream solid: $\mathrm{mp}$ ( $\mathrm{MeOH} / \mathrm{CH}_{2} \mathrm{Cl}_{2} /$ hexane $) 239-241{ }^{\circ} \mathrm{C} ;{ }^{1} \mathrm{H}$ NMR $\left[\left(\mathrm{CD}_{3}\right)_{2} \mathrm{SO}\right] \delta 8.46$ (d, $J=2.9 \mathrm{~Hz}, 1 \mathrm{H}), 8.16($ br d, $J=8.9 \mathrm{~Hz}, 2 \mathrm{H}), 8.08(\mathrm{~s}, 1 \mathrm{H}), 8.01$ $(\mathrm{d}, J=8.7 \mathrm{~Hz}, 1 \mathrm{H}), 7.67(\mathrm{dd}, J=8.8,3.0 \mathrm{~Hz}, 1 \mathrm{H}), 7.45(\mathrm{br} \mathrm{d}, J=8.1$ $\mathrm{Hz}, 2 \mathrm{H}), 5.44-5.36(\mathrm{~m}, 1 \mathrm{H}), 4.73(\mathrm{dt}, J=12.4,2.1 \mathrm{~Hz}, 1 \mathrm{H}), 4.69$ (br d, $J=12.0 \mathrm{~Hz}, 1 \mathrm{H}), 4.45(\mathrm{dd}, J=13.9,3.0 \mathrm{~Hz}, 1 \mathrm{H}), 4.39(\mathrm{br} \mathrm{d}, J$ $=14.0 \mathrm{~Hz}, 1 \mathrm{H}) ;{ }^{13} \mathrm{C} \mathrm{NMR}\left[\left(\mathrm{CD}_{3}\right)_{2} \mathrm{SO}\right] \delta 151.9,148.5\left(\mathrm{q}, J_{\mathrm{C}-\mathrm{F}}=1.4\right.$ $\mathrm{Hz}), 148.3,146.9,142.2,138.8,137.4,127.9$ (2 C), 123.9, $121.2(2 \mathrm{C})$, $121.1,120.1\left(\mathrm{q}, J_{\mathrm{C}-\mathrm{F}}=256.4 \mathrm{~Hz}\right), 118.0,67.9,66.0,46.3 ;[\alpha]_{\mathrm{D}}^{25} 6.98$ (c 1.003, DMF). Anal. Calcd for $\mathrm{C}_{18} \mathrm{H}_{13} \mathrm{~F}_{3} \mathrm{~N}_{4} \mathrm{O}_{5}: \mathrm{C}, 51.19 ; \mathrm{H}, 3.10 ; \mathrm{N}$, 13.27. Found: C, 51.33; H, 2.94; N, 13.27 .

Minimum Inhibitory Concentration Assays (MABA and LORA). These assays against $M$. $t b$ were performed according to the reported procedures. ${ }^{59,80}$ Results in Table 1 are the mean of two or three independent determinations (SD data are given in Table S1).

In Vitro Parasite Growth Inhibition Assays. The activity of test compounds against the amastigote stage of the $L$. don parasite was measured at CDRI using a mouse macrophage-based luciferase assay, performed according to the published procedures. ${ }^{26}$ Replicate assays quantifying the growth inhibitory action of compounds against $L$. inf, T. cruzi, and T. brucei and assessing any cytotoxic effects on human lung fibroblasts (MRC-5 cells) were conducted at the University of Antwerp (LMPH), as previously described; ${ }^{44}$ results in Tables $1-4$ are the mean of at least two (up to 10) independent determinations (SD data are given in Tables S1-S4). Additional assays using a wider range of VL and CL strains and clinical isolates were performed via comparable methods at $\mathrm{LMPH}^{44}$ or $\mathrm{LSHTM}^{26}$ (primary peritoneal mouse macrophages infected with cultured promastigotes were incubated at $37^{\circ} \mathrm{C}$ for $24 \mathrm{~h}$ prior to the addition of test compounds and then further incubated for either 3 or 5 days for CL or VL assays, respectively).

Solubility Determinations. Method $A$. The solid compound sample was mixed with water or $0.1 \mathrm{M} \mathrm{HCl}$ (enough to make a $2 \mathrm{mM}$ solution) in an Eppendorf tube, and the suspension was sonicated for $15 \mathrm{~min}$ and then centrifuged at $13000 \mathrm{rpm}$ for $6 \mathrm{~min}$. An aliquot of the clear supernatant was diluted 2 -fold with water (or $0.1 \mathrm{M} \mathrm{HCl}$ ), and then HPLC was performed (as described). The kinetic solubility was calculated by comparing the peak area obtained with that from a standard solution of the compound in DMSO (after allowing for varying dilution factors and injection volumes).

Method B. The thermodynamic solubility of compound $\mathrm{R}-6$ at $\mathrm{pH}$ 7.4 was measured by Syngene International Ltd. (Plot No. 2 and 3 Biocon Park, Jigani Link Road, Bangalore 560099, India). The dry powder was equilibrated with $0.1 \mathrm{M}$ phosphate buffer $(\mathrm{pH} 7.4$ ) in a glass vial at $25^{\circ} \mathrm{C}$ (water bath), shaking for $24 \mathrm{~h}$. After filtration using a $0.45 \mu \mathrm{m}$ PVDF membrane filter, the concentration of $R-6$ was determined by HPLC (Waters e2695 system, employing a $150 \mathrm{~mm} \times$ $4.6 \mathrm{~mm}$ XBridge $3.5 \mu \mathrm{m}$ reversed phase $\mathrm{C} 18$ column and isocratic elution with $50 \% \mathrm{CH}_{3} \mathrm{CN}$ in $10 \mathrm{mM}$ ammonium acetate buffer, at 1 $\mathrm{mL} / \mathrm{min}$ ), comparing the peak area obtained with that from a standard solution $(0.93 \mathrm{mM})$ in $1: 1: 2 \mathrm{EtOH} /$ water $/ \mathrm{CH}_{3} \mathrm{CN}$.

Microsomal Stability Assays. Compound 22 was tested by MDS Pharma Services (22011 30th Dr. SE, Bothell, WA 98021-4444), as described previously. ${ }^{47}$ Studies of compounds $24, S-51, S-77, R-77, S$ 81, R-81, R-84, S-89, R-89, $S-91, R-91, S-92, R-92, R-94,116,117, S-$ 151, and $S$-155 (Table 5) were run by Advinus Therapeutics Ltd. (21 and 22 Phase II, Peenya Industrial Area, Bangalore 560058, India), using a published procedure ${ }^{61}$ in which the compound concentration was $0.5 \mu \mathrm{M}$ and the incubation time was $30 \mathrm{~min}$. Additional analyses of compounds $R-1, R-6, R-69, R-74, R-84, R-89, R-91, R-92, R-94, R$ $96, R-99, R-102, R-106, R-136, R-147, R-151, R-168$, and $R-169$ were performed by WuXi AppTec (Shanghai) Co., Ltd. (288 FuTe ZhongLu, WaiGaoQiao Free Trade Zone, Shanghai 200131, China) via a reported ${ }^{25}$ method; the compound concentration was $1 \mu \mathrm{M}$, and the incubation time was $1 \mathrm{~h}$.

Distribution Coefficient. This was measured by WuXi AppTec (Shanghai) Co., Ltd. The LogD value of R-6 was found by assessing its distribution between $100 \mathrm{mM}$ phosphate buffer ( $\mathrm{pH} 7.4)$ and octanol at room temperature (final matrix contained $1 \%$ DMSO), using the shake-flask method and LC-MS/MS analysis.

Permeability Assay. The assay was performed by WuXi AppTec (Shanghai) Co., Ltd. MDCK-MDR1 cells were seeded onto polyethylene membranes in 96-well plates at a density of $2 \times 10^{5}$ cells $/ \mathrm{cm}^{2}$, giving confluent cell monolayer formation over 4-7 days. A solution of $R-6$ ( $2 \mu \mathrm{M}$ in $0.4 \%$ DMSO/HBSS buffer) was applied to the apical or basolateral side of the cell monolayer. Permeation of the compound in the $\mathrm{A}$ to $\mathrm{B}$ direction or $\mathrm{B}$ to $\mathrm{A}$ direction was assessed in triplicate over a $150 \mathrm{~min}$ incubation at $37{ }^{\circ} \mathrm{C}$ and $5 \% \mathrm{CO}_{2}(95 \%$ humidity). In addition, the efflux ratio of $\mathrm{R}-6$ was also determined. Test and reference compounds were quantified by LC-MS/MS analysis based on the peak area ratio of the analyte/internal standard.

Plasma Protein Binding Assays. The studies of $\mathbf{4}$ and R-6 were performed by WuXi AppTec (Shanghai) Co., Ltd., using equilibrium dialysis across a semipermeable membrane. Briefly, $2 \mu \mathrm{M}$ compound solutions in plasma were dialyzed against $100 \mathrm{mM}$ phosphate-buffered saline ( $\mathrm{pH} 7.4)$ on a rotating plate $(150 \mathrm{rpm})$ incubated at $37^{\circ} \mathrm{C}$ for 4 or $6 \mathrm{~h}$. Following precipitation of protein with $\mathrm{CH}_{3} \mathrm{CN}$, the amount of compound present in each compartment was quantified by LC-MS/ MS; values are the mean of triplicate determinations.

Ames Test. Compounds $R-6$ and $R-136$ (at doses of 1.5, 4, 10, 25, $64,160,400$, and $1000 \mu \mathrm{g} /$ well) were evaluated in triplicate in the Mini-Ames reverse mutation screen conducted by WuXi AppTec (Suzhou) Co., Ltd. (1318 Wuzhong Ave., Wuzhong District, Suzhou 215104, China). Two strains of Salmonella typhimurium (TA98 and TA100) were employed, in the presence and absence of metabolic activation (rat liver S9). Positive controls (2-aminoanthracene, 2nitrofluorene, and sodium azide) and a negative (DMSO solvent) control were included.

hERG Assay. The effects of compounds $R-6, R-84, R-89$, and $R$ 136 on cloned hERG potassium channels expressed in Chinese hamster ovary cells were assessed by WuXi AppTec (Shanghai) Co., Ltd., using the automated patch clamp method. Six concentrations $(0.12,0.37,1.11,3.33,10$, and $30 \mu \mathrm{M}$ ) were tested (at room temperature), and at least three replicates were obtained for each.

CYP3A4 Inhibition Assay. This work was performed by WuXi AppTec (Shanghai) Co., Ltd. Compound R-6 (at concentrations of 1 and $10 \mu \mathrm{M})$ was incubated with NADPH-fortified pooled HLM $(0.2$ 
$\mathrm{mg} / \mathrm{mL})$ and testosterone $(50 \mu \mathrm{M})$ in phosphate buffer $(100 \mathrm{mM})$ at $37^{\circ} \mathrm{C}$ for $10 \mathrm{~min}$. Following quenching with $\mathrm{CH}_{3} \mathrm{CN}$, samples were analyzed for the formation of $6 \beta$-hydroxytestosterone by LC-MS/MS, and the percentage inhibition was determined (ketoconazole was the positive control, and tolbutamide was used as an internal standard).

In Vivo Experiments. All animal experiments were performed according to institutional ethical guidelines for animal care. Mouse model studies (LSHTM) were conducted under license (PPL X20014A54), according to UK Home Office regulations, Animals (Scientific Procedures) Act 1986, and European Directive 2010/63/ $\mathrm{EU}$, and hamster studies (LMPH) were approved by the ethical committee of the University of Antwerp (UA-ECD 2010-17).

Acute VL Infection Assay (mouse model, LSHTM). Test compounds were orally administered once per day for 5 days consecutively to groups of five female BALB/c mice infected with $2 \times$ $10^{7} \mathrm{~L}$. don amastigotes, with treatment commencing 1 week postinfection, as described previously. ${ }^{26}$ Miltefosine and AmBisome were positive controls, and parasite burdens were determined from impression smears of liver sections. Efficacy was expressed as the mean percentage reduction in parasite load for treated mice in comparison to untreated (vehicle-only) controls (SD data are provided in Table S5). Derived $\mathrm{ED}_{50}$ values (with $95 \%$ confidence limits, as specified in Table S5) were obtained from GraphPad Prism 6 software, using a fourparametric sigmoidal variable slope dose-response curve.

Chronic VL Infection Assay (hamster model, LMPH). Golden hamsters (weighing 75-80 g) were infected with $2 \times 10^{7} \mathrm{~L}$. inf amastigotes, and 21 days postinfection, treatment groups of six animals each were treated orally twice per day with test compounds (formulated in PEG-400) for 5 days consecutively. Parasite burdens in three target organs (liver, spleen, and bone marrow) were determined by microscopic evaluation of impression smears (stained with Giemsa), and efficacy was expressed as the mean percentage parasite load reduction for treated hamsters in comparison to untreated (vehicle-only) controls (SD data are given in Table S6). Miltefosine was included as a reference drug in all experiments.

Mouse Pharmacokinetics. Testing of compounds 24, 116, and 117 was executed by Advinus Therapeutics Ltd., according to a published protocol. ${ }^{61}$ Briefly, compounds were administered to groups of male Swiss albino mice; intravenous dosing (at $1 \mathrm{mg} / \mathrm{kg}$ ) employed a solution vehicle comprising 20\% NMP and 40\% PEG-400 in 100 $\mathrm{mM}$ citrate buffer $(\mathrm{pH} 3)$, while oral dosing (at $25 \mathrm{mg} / \mathrm{kg}$ ) was as a suspension in $0.5 \%$ carboxymethylcellulose (CMC) and $0.08 \%$ Tween 80 in water. Samples derived from plasma (at 0.083 for iv only, 0.25 , $0.5,1,2,4,6,8,10,24$, and $48 \mathrm{~h}$ ) were centrifuged prior to analysis by LC-MS/MS, and the PK parameters were determined using Phoenix WinNonlin software (version 5.2). The remaining compounds ( $R-6$, $R-84, R-89$, and $R-136$ ) were assessed by WuXi AppTec (Shanghai) Co., Ltd.; in this case, oral dosing of female $\mathrm{BALB} / \mathrm{c}$ mice occurred at $40-50 \mathrm{mg} / \mathrm{kg}$ in PEG-400 (sampling at $0.25,1,2,4,8$, and $24 \mathrm{~h}$ ), and the PK data were obtained using WinNonlin software (version 6.2) following similar LC-MS/MS analysis.

Rat and Hamster Pharmacokinetics. All studies were conducted in fasted animals (either male Sprague-Dawley rats or female golden Syrian hamsters) by WuXi AppTec (Shanghai) Co., Ltd. Intravenous dosing (at $1 \mathrm{mg} / \mathrm{kg}$ for rats and $2 \mathrm{mg} / \mathrm{kg}$ for hamsters) utilized a solution formulation of $20 \%$ NMP and $40 \%$ PEG-400 in citrate buffer ( $\mathrm{pH} 3$ ). In rats, oral dosing (at $40-50 \mathrm{mg} / \mathrm{kg}$ ) was as a suspension in $0.08 \%$ Tween 80 and $0.5 \%$ CMC in water, whereas PEG-400 was the vehicle employed for oral dosing in hamsters (at $40-50 \mathrm{mg} / \mathrm{kg}$ ). Plasma samples (at 0.083 for iv only, $0.25,0.5,1,2,4,8$, and $24 \mathrm{~h}$ ) were analyzed by LC-MS/MS, and the PK parameters were calculated using Phoenix WinNonlin software (version 6.3).

\section{ASSOCIATED CONTENT}

\section{S Supporting Information}

The Supporting Information is available free of charge on the ACS Publications website at DOI: 10.1021/acs.jmedchem.7b01581.
Additional biological assay data, synthetic schemes, graphs of PK data, experimental procedures and characterizations for compounds, combustion analytical data, and representative NMR spectra (PDF) Molecular formula strings spreadsheet (CSV)

\section{AUTHOR INFORMATION}

\section{Corresponding Author}

*E-mail: am.thompson@auckland.ac.nz. Phone: (+649) 9236145. Fax: $(+649)$ 373-7502.

ORCID

Andrew M. Thompson: 0000-0003-2593-8559

William A. Denny: 0000-0001-7997-1843

Notes

The authors declare no competing financial interest.

\section{ACKNOWLEDGMENTS}

The authors thank the Global Alliance for TB Drug Development (TB Alliance) and the Drugs for Neglected Diseases initiative (DNDi) for financial support through collaborative research agreements. For this project, the TB Alliance acknowledges grant funding from the Bill \& Melinda Gates Foundation (OPP40827), while DND $i$ received financial support from the following donors: Department for International Development (DFID), of the U.K.; Federal Ministry of Education and Research (BMBF), through KfW Germany; Directorate-General for International Cooperation (DGIS), of The Netherlands; Bill \& Melinda Gates Foundation (grant OPP48262); and Médecins Sans Frontières (MSF) International. The donors had no role in study design, data collection and analysis, decision to publish, or preparation of the manuscript. The authors also thank Drs. Jakir Pinjari and Rao Mukkavilli (Advinus Therapeutics Ltd., Bangalore, India) for some PK data, Sisira Kumara (ACSRC) for the kinetic solubility measurements, and Dr. Beatrice Bonnet (DNDi) for providing some new data and comments on the large scale synthesis of the lead.

\section{ABBREVIATIONS USED}

VL, visceral leishmaniasis; TPP, target product profile; $M . t b, M$. tuberculosis; TB, tuberculosis; L. inf, L. infantum; L. don, $L$. donovani; HLM, human liver microsomes; PK, pharmacokinetic; DMPK, drug metabolism and pharmacokinetic; MLM, mouse liver microsomes; PD, pharmacodynamic; HREIMS, high-resolution electron impact mass spectrometry; HRCIMS, high-resolution chemical ionization mass spectrometry; HRFABMS, high-resolution fast atom bombardment mass spectrometry; HRESIMS, high-resolution electrospray ionization mass spectrometry; APCI MS, atmospheric-pressure chemical ionization mass spectrometry; DIPEA, $N, N$-diisopropylethylamine; NMM, N-methylmorpholine; CMC, carboxymethylcellulose; MIL, miltefosine; SD, standard deviation

\section{REFERENCES}

(1) Leishmaniasis. World Health Organization: Geneva, 2017. http://www.who.int/mediacentre/factsheets/fs375/en/ (accessed June 22, 2017).

(2) Karimi, A.; Alborzi, A.; Amanati, A. Visceral leishmaniasis: An update and literature review. Archives of Pediatric Infectious Diseases 2016, 4 (3), e31612.

(3) den Boer, M.; Argaw, D.; Jannin, J.; Alvar, J. Leishmaniasis impact and treatment access. Clin. Microbiol. Infect. 2011, 17, 1471-1477. 
(4) Al-Salem, W.; Herricks, J. R.; Hotez, P. J. A review of visceral leishmaniasis during the conflict in South Sudan and the consequences for East African countries. Parasites Vectors 2016, 9, 460.

(5) Sunyoto, T.; Potet, J.; Boelaert, M. Visceral leishmaniasis in Somalia: A review of epidemiology and access to care. PLoS Neglected Trop. Dis. 2017, 11 (3), e0005231.

(6) Kimutai, R.; Musa, A. M.; Njoroge, S.; Omollo, R.; Alves, F.; Hailu, A.; Khalil, E. A. G.; Diro, E.; Soipei, P.; Musa, B.; Salman, K.; Ritmeijer, K.; Chappuis, F.; Rashid, J.; Mohammed, R.; Jameneh, A.; Makonnen, E.; Olobo, J.; Okello, L.; Sagaki, P.; Strub, N.; Ellis, S.; Alvar, J.; Balasegaram, M.; Alirol, E.; Wasunna, M. Safety and effectiveness of sodium stibogluconate and paromomycin combination for the treatment of visceral leishmaniasis in Eastern Africa: Results from a pharmacovigilance programme. Clin. Drug Invest. 2017, 37, 259-272.

(7) Wasunna, M.; Njenga, S.; Balasegaram, M.; Alexander, N.; Omollo, R.; Edwards, T.; Dorlo, T. P. C.; Musa, B.; Ali, M. H. S.; Elamin, M. Y.; Kirigi, G.; Juma, R.; Kip, A. E.; Schoone, G. J.; Hailu, A.; Olobo, J.; Ellis, S.; Kimutai, R.; Wells, S.; Khalil, E. A. G.; Strub Wourgaft, N.; Alves, F.; Musa, A. Efficacy and safety of AmBisome in combination with sodium stibogluconate or miltefosine and miltefosine monotherapy for African visceral leishmaniasis: Phase II randomized trial. PLoS Neglected Trop. Dis. 2016, 10 (9), e0004880.

(8) Fexinidazole/Miltefosine Combination (VL). DNDi, 2016. https://www.dndi.org/diseases-projects/portfolio/completedprojects/fexinidazole-vl/ (accessed June 22, 2017).

(9) Singh, N.; Mishra, B. B.; Bajpai, S.; Singh, R. K.; Tiwari, V. K. Natural product based leads to fight against leishmaniasis. Bioorg. Med. Chem. 2014, 22, 18-45.

(10) Target Product Profile for Visceral Leishmaniasis. DNDi, 2017. https://www.dndi.org/diseases-projects/leishmaniasis/tpp-vl/ (accessed December 7, 2017).

(11) Nagle, A. S.; Khare, S.; Kumar, A. B.; Supek, F.; Buchynskyy, A.; Mathison, C. J. N.; Chennamaneni, N. K.; Pendem, N.; Buckner, F. S.; Gelb, M. H.; Molteni, V. Recent developments in drug discovery for leishmaniasis and human African trypanosomiasis. Chem. Rev. 2014, $114,11305-11347$.

(12) Field, M. C.; Horn, D.; Fairlamb, A. H.; Ferguson, M. A. J.; Gray, D. W.; Read, K. D.; De Rycker, M.; Torrie, L. S.; Wyatt, P. G.; Wyllie, S.; Gilbert, I. H. Anti-trypanosomatid drug discovery: an ongoing challenge and a continuing need. Nat. Rev. Microbiol. 2017, $15,217-231$.

(13) Don, R; Ioset, J.-R. Screening strategies to identify new chemical diversity for drug development to treat kinetoplastid infections. Parasitology 2014, 141, 140-146.

(14) Liévin-Le Moal, V.; Loiseau, P. M. Leishmania hijacking of the macrophage intracellular compartments. FEBS J. 2016, 283, 598-607.

(15) Naderer, T.; Vince, J. E.; McConville, M. J. Surface determinants of Leishmania parasites and their role in infectivity in the mammalian host. Curr. Mol. Med. 2004, 4, 649-665.

(16) Katsuno, K.; Burrows, J. N.; Duncan, K.; Hooft van Huijsduijnen, R.; Kaneko, T.; Kita, K.; Mowbray, C. E.; Schmatz, D.; Warner, P.; Slingsby, B. T. Hit and lead criteria in drug discovery for infectious diseases of the developing world. Nat. Rev. Drug Discovery 2015, 14, 751-758.

(17) Burrows, J. N.; Elliott, R. L.; Kaneko, T.; Mowbray, C. E.; Waterson, $\mathrm{D}$. The role of modern drug discovery in the fight against neglected and tropical diseases. MedChem Comm 2014, 5, 688-700.

(18) Mowbray, C. E.; Braillard, S.; Speed, W.; Glossop, P. A.; Whitlock, G. A.; Gibson, K. R.; Mills, J. E. J.; Brown, A. D.; Gardner, J. M. F.; Cao, Y.; Hua, W.; Morgans, G. L.; Feijens, P.-B.; Matheeussen, A.; Maes, L. J. Novel amino-pyrazole ureas with potent in vitro and in vivo antileishmanial activity. J. Med. Chem. 2015, 58, 9615-9624.

(19) Khare, S.; Nagle, A. S.; Biggart, A.; Lai, Y. H.; Liang, F.; Davis, L. C.; Barnes, S. W.; Mathison, C. J. N.; Myburgh, E.; Gao, M.-Y.; Gillespie, J. R.; Liu, X.; Tan, J. L.; Stinson, M.; Rivera, I. C.; Ballard, J.; Yeh, V.; Groessl, T.; Federe, G.; Koh, H. X. Y.; Venable, J. D.; Bursulaya, B.; Shapiro, M.; Mishra, P. K.; Spraggon, G.; Brock, A.; Mottram, J. C.; Buckner, F. S.; Rao, S. P. S.; Wen, B. G.; Walker, J. R.;
Tuntland, T.; Molteni, V.; Glynne, R. J.; Supek, F. Proteasome inhibition for treatment of leishmaniasis, Chagas disease and sleeping sickness. Nature 2016, 537, 229-233.

(20) Mukherjee, T.; Boshoff, H. Nitroimidazoles for the treatment of TB: past, present and future. Future Med. Chem. 2011, 3, 1427-1454.

(21) Diacon, A. H.; Dawson, R.; du Bois, J.; Narunsky, K.; Venter, A.; Donald, P. R.; van Niekerk, C.; Erondu, N.; Ginsberg, A. M.; Becker, P.; Spigelman, M. K. Phase II dose-ranging trial of the early bactericidal activity of PA-824. Antimicrob. Agents Chemother. 2012, 56, 3027-3031.

(22) Murray, S.; Mendel, C.; Spigelman, M. TB Alliance regimen development for multidrug-resistant tuberculosis. International Journal of Tuberculosis and Lung Disease 2016, 20 (Suppl. 1), S38-S41.

(23) Upton, A. M.; Cho, S.; Yang, T. J.; Kim, Y.; Wang, Y.; Lu, Y.; Wang, B.; Xu, J.; Mdluli, K.; Ma, Z.; Franzblau, S. G. In vitro and in vivo activities of the nitroimidazole TBA-354 against Mycobacterium tuberculosis. Antimicrob. Agents Chemother. 2015, 59, 136-144.

(24) Thompson, A. M.; O’Connor, P. D.; Marshall, A. J.; Yardley, V.; Maes, L.; Gupta, S.; Launay, D.; Braillard, S.; Chatelain, E.; Franzblau, S. G.; Wan, B.; Wang, Y.; Ma, Z.; Cooper, C. B.; Denny, W. A. 7Substituted 2-nitro-5,6-dihydroimidazo[2,1-b][1,3] oxazines: novel antitubercular agents lead to a new preclinical candidate for visceral leishmaniasis. J. Med. Chem. 2017, 60, 4212-4233.

(25) Thompson, A. M.; O'Connor, P. D.; Blaser, A.; Yardley, V.; Maes, L.; Gupta, S.; Launay, D.; Martin, D.; Franzblau, S. G.; Wan, B.; Wang, Y.; Ma, Z.; Denny, W. A. Repositioning antitubercular 6-nitro2,3-dihydroimidazo[2,1-b][1,3] oxazoles for neglected tropical diseases: structure-activity studies on a preclinical candidate for visceral leishmaniasis. J. Med. Chem. 2016, 59, 2530-2550.

(26) Gupta, S.; Yardley, V.; Vishwakarma, P.; Shivahare, R.; Sharma, B.; Launay, D.; Martin, D.; Puri, S. K. Nitroimidazo-oxazole compound DNDI-VL-2098: an orally effective preclinical drug candidate for the treatment of visceral leishmaniasis. J. Antimicrob. Chemother. 2015, 70, $518-527$.

(27) Wyllie, S.; Roberts, A. J.; Norval, S.; Patterson, S.; Foth, B. J.; Berriman, M.; Read, K. D.; Fairlamb, A. H. Activation of bicyclic nitrodrugs by a novel nitroreductase (NTR2) in Leishmania. PLoS Pathog. 2016, 12 (11), e1005971.

(28) Patterson, S.; Wyllie, S.; Norval, S.; Stojanovski, L.; Simeons, F. R. C.; Auer, J. L.; Osuna-Cabello, M.; Read, K. D.; Fairlamb, A. H. The anti-tubercular drug delamanid as a potential oral treatment for visceral leishmaniasis. eLife 2016, 5, e09744.

(29) Thompson, A. M.; Denny, W. A.; Blaser, A.; Ma, Z. Nitroimidazooxazine and Nitroimidazooxazole Analogues and Their Uses. Patent WO 2011/014776 Al, 2011, and U.S. Patent 8293734 B2, 2012.

(30) Thompson, A. M.; Blaser, A.; Palmer, B. D.; Franzblau, S. G.; Wan, B.; Wang, Y.; Ma, Z.; Denny, W. A. Biarylmethoxy 2nitroimidazooxazine antituberculosis agents: Effects of proximal ring substitution and linker reversal on metabolism and efficacy. Bioorg. Med. Chem. Lett. 2015, 25, 3804-3809.

(31) Li, X.; Manjunatha, U. H.; Goodwin, M. B.; Knox, J. E.; Lipinski, C. A.; Keller, T. H.; Barry, C. E.; Dowd, C. S. Synthesis and antitubercular activity of 7-(R)- and 7-(S)-methyl-2-nitro-6-(S)-(4(trifluoromethoxy)benzyloxy)-6,7-dihydro-5H-imidazo[2,1-b][1,3]oxazines, analogues of PA-824. Bioorg. Med. Chem. Lett. 2008, 18, $2256-2262$.

(32) Baker, W. R.; Shaopei, C.; Keeler, E. L. Nitro-[2,1b]imidazopyran Compounds and Antibacterial Uses Thereof. U.S. Patent 6087358, 2000.

(33) Hirata, M.; Fujimoto, R.; Mikami, M. Preparation of 2Methylglycidyl Ethers from 3-Halogeno-2-methyl-1,2-propanediols or 2-Methylepihalohydrins. Patent JP 2007297330, 2007.

(34) Elbert, B. L.; Lim, D. S. W.; Gudmundsson, H. G.; O’Hanlon, J. A.; Anderson, E. A. Synthesis of cyclic alkenylsiloxanes by semihydrogenation: a stereospecific route to $(Z)$-alkenyl polyenes. Chem. Eur. J. 2014, 20, 8594-8598.

(35) Ginsberg, A. M.; Laurenzi, M. W.; Rouse, D. J.; Whitney, K. D.; Spigelman, M. K. Safety, tolerability, and pharmacokinetics of PA-824 
in healthy subjects. Antimicrob. Agents Chemother. 2009, 53, 37203725.

(36) Thompson, A. M.; Marshall, A. J.; Maes, L.; Yarlett, N.; Bacchi, C. J.; Gaukel, E.; Wring, S. A.; Launay, D.; Braillard, S.; Chatelain, E.; Mowbray, C. E.; Denny, W. A. Assessment of a pretomanid analogue library for African trypanosomiasis: Hit-to-lead studies on 6substituted 2-nitro-6,7-dihydro-5H-imidazo[2,1-b][1,3]thiazine 8-oxides. Bioorg. Med. Chem. Lett. 2018, 28, 207-213.

(37) Gurumurthy, M.; Mukherjee, T.; Dowd, C. S.; Singh, R.; Niyomrattanakit, P.; Tay, J. A.; Nayyar, A.; Lee, Y. S.; Cherian, J.; Boshoff, H. I.; Dick, T.; Barry, C. E., III; Manjunatha, U. H. Substrate specificity of the deazaflavin-dependent nitroreductase from Mycobacterium tuberculosis responsible for the bioreductive activation of bicyclic nitroimidazoles. FEBS J. 2012, 279, 113-125.

(38) Patterson, S.; Wyllie, S.; Stojanovski, L.; Perry, M. R.; Simeons, F. R. C.; Norval, S.; Osuna-Cabello, M.; De Rycker, M.; Read, K. D.; Fairlamb, A. H. The $R$ enantiomer of the antitubercular drug PA-824 as a potential oral treatment for visceral leishmaniasis. Antimicrob. Agents Chemother. 2013, 57, 4699-4706.

(39) Kmentova, I.; Sutherland, H. S.; Palmer, B. D.; Blaser, A.; Franzblau, S. G.; Wan, B.; Wang, Y.; Ma, Z.; Denny, W. A.; Thompson, A. M. Synthesis and structure-activity relationships of azaand diazabiphenyl analogues of the antitubercular drug (6S)-2-nitro-6$\{[4$-(trifluoromethoxy)benzyl $]$ oxy $\}$-6,7-dihydro- $5 H$-imidazo[2,1-b][1,3] oxazine (PA-824). J. Med. Chem. 2010, 53, 8421-8439.

(40) Thompson, A. M.; Blaser, A.; Anderson, R. F.; Shinde, S. S.; Franzblau, S. G.; Ma, Z.; Denny, W. A.; Palmer, B. D. Synthesis, reduction potentials, and antitubercular activity of ring $\mathrm{A} / \mathrm{B}$ analogues of the bioreductive drug (6S)-2-nitro-6-\{[4-(trifluoromethoxy)benzyl $]$ oxy $\}$-6,7-dihydro-5H-imidazo[2,1-b][1,3] oxazine (PA-824). J. Med. Chem. 2009, 52, 637-645.

(41) Bom, D.; Curran, D. P.; Kruszewski, S.; Zimmer, S. G.; Thompson Strode, J.; Kohlhagen, G.; Du, W.; Chavan, A. J.; Fraley, K. A.; Bingcang, A. L.; Latus, L. J.; Pommier, Y.; Burke, T. G. The novel silatecan 7-tert-butyldimethylsilyl-10-hydroxycamptothecin displays high lipophilicity, improved human blood stability, and potent anticancer activity. J. Med. Chem. 2000, 43, 3970-3980.

(42) Thompson, A. M.; Sutherland, H. S.; Palmer, B. D.; Kmentova, I.; Blaser, A.; Franzblau, S. G.; Wan, B.; Wang, Y.; Ma, Z.; Denny, W. A. Synthesis and structure-activity relationships of varied ether linker analogues of the antitubercular drug (6S)-2-nitro-6-\{[4(trifluoromethoxy)benzyl] oxy\}-6,7-dihydro-5H-imidazo[2,1-b][1,3]oxazine (PA-824). J. Med. Chem. 2011, 54, 6563-6585.

(43) Alberati-Giani, D.; Jolidon, S.; Narquizian, R.; Nettekoven, M. H.; Norcross, R. D.; Pinard, E.; Stalder, H. Preparation of 1-(2Aminobenzoyl)-piperazine Derivatives as Glycine Transporter 1 (GlyT-1) Inhibitors for Treating Psychoses. Patent WO 2005023260 A1, 2005.

(44) Kaiser, M.; Maes, L.; Tadoori, L. P.; Spangenberg, T.; Ioset, J.-R. Repurposing of the open access malaria box for kinetoplastid diseases identifies novel active scaffolds against trypanosomatids. J. Biomol. Screening 2015, 20, 634-645.

(45) Siqueira-Neto, J. L.; Song, O.-R.; Oh, H.; Sohn, J.-H.; Yang, G.; Nam, J.; Jang, J.; Cechetto, J.; Lee, C. B.; Moon, S.; Genovesio, A.; Chatelain, E.; Christophe, T.; Freitas-Junior, L. H. Antileishmanial high-throughput drug screening reveals drug candidates with new scaffolds. PLoS Neglected Trop. Dis. 2010, 4 (5), e675.

(46) Palmer, B. D.; Sutherland, H. S.; Blaser, A.; Kmentova, I.; Franzblau, S. G.; Wan, B.; Wang, Y.; Ma, Z.; Denny, W. A.; Thompson, A. M. Synthesis and structure-activity relationships for extended side chain analogues of the antitubercular drug (6S)-2-nitro6- $\{[4$-(trifluoromethoxy) benzyl $]$ oxy $\}-6,7-$ dihydro- $5 H$-imidazo $[2,1-b]$ $[1,3]$ oxazine (PA-824). J. Med. Chem. 2015, 58, 3036-3059.

(47) Palmer, B. D.; Thompson, A. M.; Sutherland, H. S.; Blaser, A.; Kmentova, I.; Franzblau, S. G.; Wan, B.; Wang, Y.; Ma, Z.; Denny, W. A. Synthesis and structure-activity studies of biphenyl analogues of the tuberculosis drug (6S)-2-nitro-6-\{[4-(trifluoromethoxy)benzyl $]$ oxy $\}$ 6,7-dihydro-5H-imidazo[2,1-b][1,3] oxazine (PA-824). J. Med. Chem. 2010, 53, 282-294.
(48) Blaser, A.; Palmer, B. D.; Sutherland, H. S.; Kmentova, I.; Franzblau, S. G.; Wan, B.; Wang, Y.; Ma, Z.; Thompson, A. M.; Denny, W. A. Structure-activity relationships for amide-, carbamate-, and urea-linked analogues of the tuberculosis drug (6S)-2-nitro-6-\{[4(trifluoromethoxy)benzyl] oxy\}-6,7-dihydro-5H-imidazo[2,1-b][1,3]oxazine (PA-824). J. Med. Chem. 2012, 55, 312-326.

(49) Sutherland, H. S.; Blaser, A.; Kmentova, I.; Franzblau, S. G.; Wan, B.; Wang, Y.; Ma, Z.; Palmer, B. D.; Denny, W. A.; Thompson, A. M. Synthesis and structure-activity relationships of antitubercular 2nitroimidazooxazines bearing heterocyclic side chains. J. Med. Chem. 2010, 53, 855-866.

(50) Kataoka, M.; Fukahori, M.; Ikemura, A.; Kubota, A.; Higashino, H.; Sakuma, S.; Yamashita, S. Effects of gastric $\mathrm{pH}$ on oral drug absorption: In vitro assessment using a dissolution/permeation system reflecting the gastric dissolution process. Eur. J. Pharm. Biopharm. 2016, 101, 103-111.

(51) Rouault, E.; Lecoeur, H.; Meriem, A. B.; Minoprio, P.; Goyard, S.; Lang, T. Imaging visceral leishmaniasis in real time with golden hamster model: Monitoring the parasite burden and hamster transcripts to further characterize the immunological responses of the host. Parasitol. Int. 2017, 66, 933-939.

(52) Yao, X.; Anderson, D. L.; Ross, S. A.; Lang, D. G.; Desai, B. Z.; Cooper, D. C.; Wheelan, P.; McIntyre, M. S.; Bergquist, M. L.; MacKenzie, K. I.; Becherer, J. D.; Hashim, M. A. Predicting QT prolongation in humans during early drug development using hERG inhibition and an anaesthetized guinea-pig model. Br. J. Pharmacol. 2008, 154, 1446-1456.

(53) Nwaka, S.; Ramirez, B.; Brun, R.; Maes, L.; Douglas, F.; Ridley, R. Advancing drug innovation for neglected diseases - criteria for lead progression. PLoS Neglected Trop. Dis. 2009, 3 (8), e440.

(54) Freitas-Junior, L. H.; Chatelain, E.; Kim, H. A.; Siqueira-Neto, J. L. Visceral leishmaniasis treatment: What do we have, what do we need and how to deliver it? Int. J. Parasitol.: Drugs Drug Resist. 2012, 2, $11-19$.

(55) Tweats, D.; Bourdin Trunz, B.; Torreele, E. Genotoxicity profile of fexinidazole - a drug candidate in clinical development for human African trypanomiasis (sleeping sickness). Mutagenesis 2012, 27, 523532.

(56) Pilkington, L. I.; Barker, D. Total synthesis of (-)-isoamericanin A and (+)-isoamericanol A. Eur. J. Org. Chem. 2014, 2014, 1037-1046.

(57) Andresen, T. L.; Jensen, S. S.; Madsen, R.; Jørgensen, K. Synthesis and biological activity of anticancer ether lipids that are specifically released by phospholipase $\mathrm{A}_{2}$ in tumor tissue. J. Med. Chem. 2005, 48, 7305-7314.

(58) Patterson, S.; Wyllie, S. Nitro drugs for the treatment of trypanosomatid diseases: past, present, and future prospects. Trends Parasitol. 2014, 30, 289-298.

(59) Falzari, K.; Zhu, Z.; Pan, D.; Liu, H.; Hongmanee, P.; Franzblau, S. G. In vitro and in vivo activities of macrolide derivatives against Mycobacterium tuberculosis. Antimicrob. Agents Chemother. 2005, 49, $1447-1454$.

(60) Cho, S. H.; Warit, S.; Wan, B.; Hwang, C. H.; Pauli, G. F.; Franzblau, S. G. Low-oxygen-recovery assay for high-throughput screening of compounds against nonreplicating Mycobacterium tuberculosis. Antimicrob. Agents Chemother. 2007, 51, 1380-1385.

(61) Mukkavilli, R.; Pinjari, J.; Patel, B.; Sengottuvelan, S.; Mondal, S.; Gadekar, A.; Verma, M.; Patel, J.; Pothuri, L.; Chandrashekar, G.; Koiram, P.; Harisudhan, T.; Moinuddin, A.; Launay, D.; Vachharajani, N.; Ramanathan, V.; Martin, D. In vitro metabolism, disposition, preclinical pharmacokinetics and prediction of human pharmacokinetics of DNDI-VL-2098, a potential oral treatment for Visceral Leishmaniasis. Eur. J. Pharm. Sci. 2014, 65, 147-155.

(62) Hendrickx, S.; Van den Kerkhof, M.; Mabille, D.; Cos, P.; Delputte, P.; Maes, L.; Caljon, G. Combined treatment of miltefosine and paromomycin delays the onset of experimental drug resistance in Leishmania infantum. PLoS Neglected Trop. Dis. 2017, 11 (5), e0005620. 